\title{
Quantification of Right Ventricular Function in Pulmonary Hypertension using Cardiac PET Images
}

\author{
By
}

Simisani Takobana

A thesis submitted to the Faculty of Graduate and Postdoctoral Affairs in partial fulfillment of the requirements for the degree of

Master of Applied Science

in

Biomedical Engineering

Carleton University

Ottawa, Ontario

(C) 2012, Simisani Takobana 
Library and Archives

Canada

Published Heritage

Branch

395 Wellington Street

Ottawa ON K1A ON4

Canada
Bibliothèque et

Archives Canada

Direction du

Patrimoine de l'édition

395 , rue Wellington

Ottawa ON K1A ON4

Canada
Your file Votre référence

ISBN: 978-0-494-94274-1

Our file Notre référence

ISBN: 978-0-494-94274-1
NOTICE:

The author has granted a nonexclusive license allowing Library and Archives Canada to reproduce, publish, archive, preserve, conserve, communicate to the public by telecommunication or on the Internet, loan, distrbute and sell theses worldwide, for commercial or noncommercial purposes, in microform, paper, electronic and/or any other formats.

The author retains copyright ownership and moral rights in this thesis. Neither the thesis nor substantial extracts from it may be printed or otherwise reproduced without the author's permission.
AVIS:

L'auteur a accordé une licence non exclusive permettant à la Bibliothèque et Archives Canada de reproduire, publier, archiver, sauvegarder, conserver, transmettre au public par télécommunication ou par l'Internet, prêter, distribuer et vendre des thèses partout dans le monde, à des fins commerciales ou autres, sur support microforme, papier, électronique et/ou autres formats.

L'auteur conserve la propriété du droit d'auteur et des droits moraux qui protege cette thèse. $\mathrm{Ni}$ la thèse ni des extraits substantiels de celle-ci ne doivent être imprimés ou autrement reproduits sans son autorisation.
In compliance with the Canadian Privacy Act some supporting forms may have been removed from this thesis.

While these forms may be included in the document page count, their removal does not represent any loss of content from the thesis.
Conformément à la loi canadienne sur la protection de la vie privée, quelques formulaires secondaires ont été enlevés de cette thèse.

Bien que ces formulaires aient inclus dans la pagination, il n'y aura aucun contenu manquant. 


\section{Abstract}

Pulmonary hypertension $(\mathrm{PH})$ is associated with the enlargement of the right ventricle (RV) and leads to a decline in RV function. The life expectancy for a patient with PH is about 3 years. Therefore, analysis of RV size and its ventricular function may aid diagnosis and treatment planning of PH. Ideally, analysis of RV function would provide an image of the energy use (via glucose uptake) across the RV myocardium, and can evaluate disease progression and response to therapy. The goal of this research is to develop, test and validate a software utility that automatically determines the physiologic and anatomic functions of the RV from Positron Emission Tomography (PET) images.

A partially automatic software system was developing based on a spline model with 12 control points and 13 degrees of freedom was developed. The system was then validated and characterized using data from different populations including non-PH patients, $\mathrm{PH}$ patients, and respective animal models. The degree of automation and operator variability when using the model was analyzed to establish the model's performance and behavior. The utility was also evaluated for accuracy when measuring RV cavity volumes and ejection fractions. Results show the region of interest (ROI) of the RV can be semiautomatically segmented from PET images for a wide range of RV morphologies including hypertrophic and normal cases.

The developed utility may be useful to diagnose and monitor disease in clinic and to guide therapy for improved prognosis and quality of life of patients suffering of RV related ailments. 


\section{Acknowledgements}

It is with immense gratitude that I acknowledge the extraordinary support and help of my supervisors, Professor Andy Adler and Dr. Ran Klein throughout this research work. 


\section{Table of Contents}

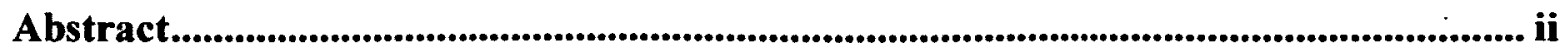

Acknowledgements .............................................................................................................................. iii

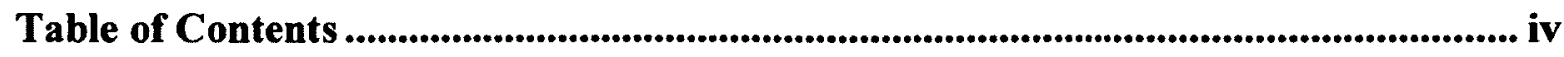

List of Tables .............................................................................................................. vii

List of Illustrations......................................................................................................................... viii

List of Abbreviations ........................................................................................................................ xiii

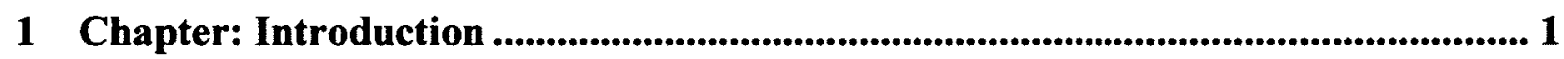

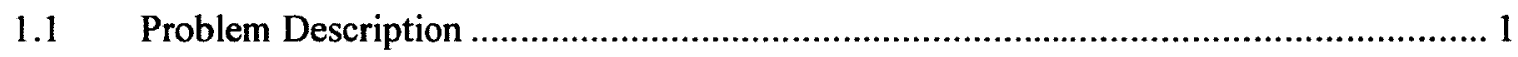

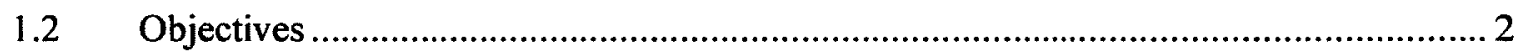

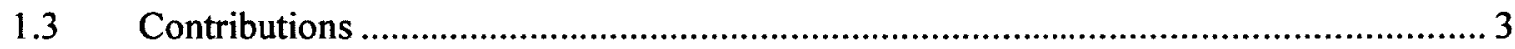

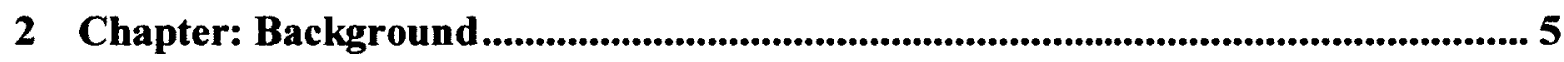

2.1 Positron Emission Tomography (PET) and Single Photon Emission Tomography

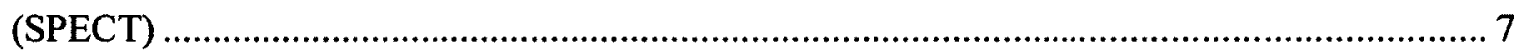

2.2 Comparison between PET and SPECT …………............................................. 10

2.2.1 Perfusion Imaging with PET ........................................................................ 10

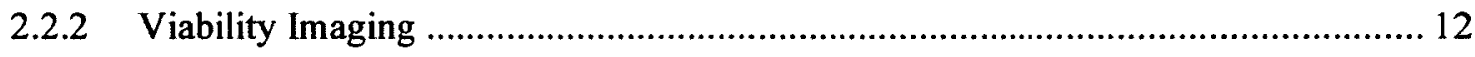

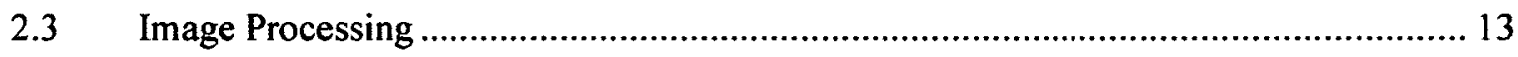

$2.4 \quad$ Image Reorientation ..................................................................................... 14

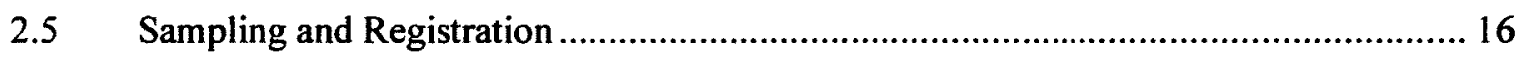

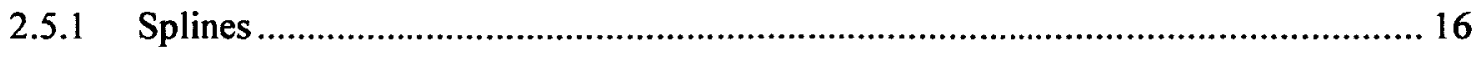

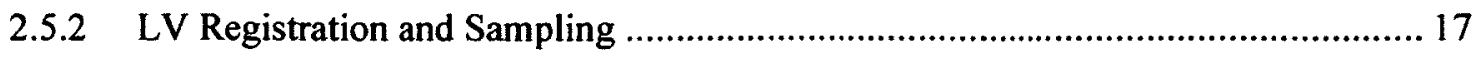

2.6 Analysis of dynamic PET images ...................................................................... 19

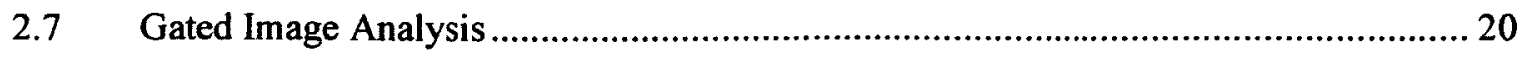

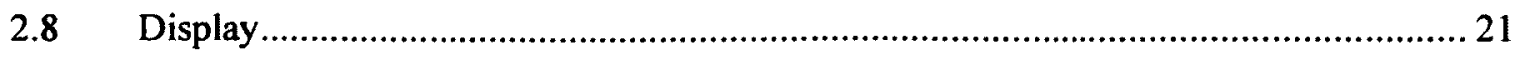


2.9 Other Work on Right Ventricular Imaging and Quantification ............................. 24

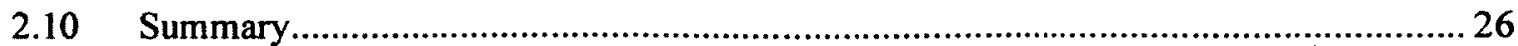

3 Chapter: Software Development ................................................................ 27

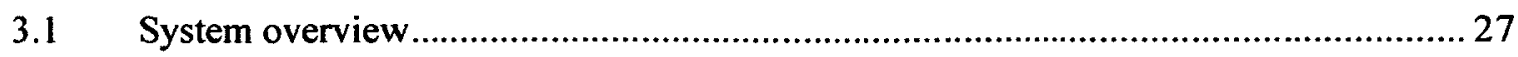

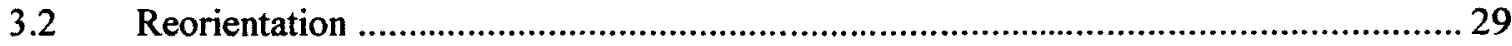

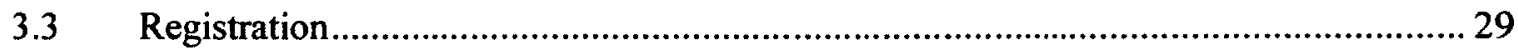

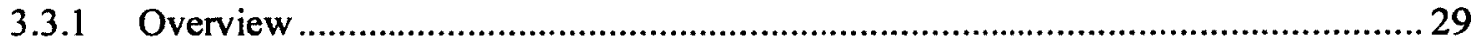

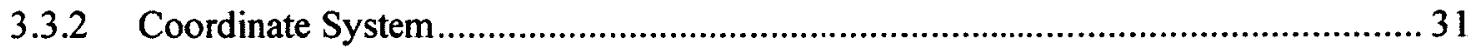

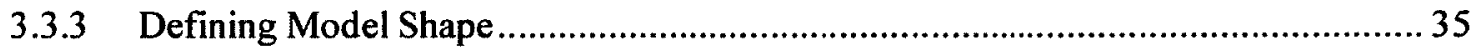

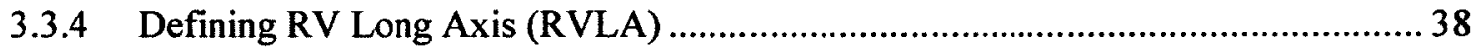

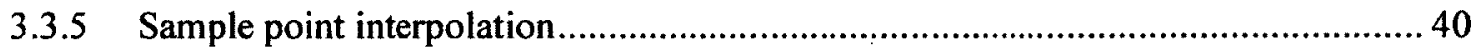

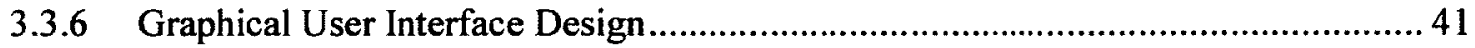

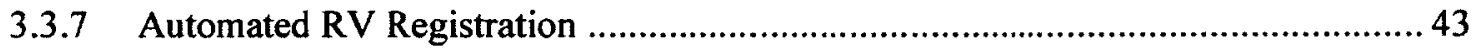

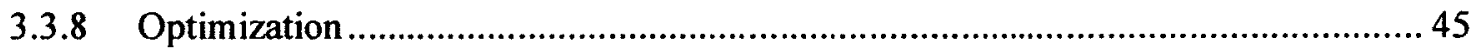

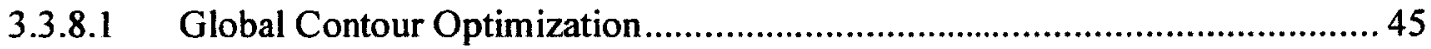

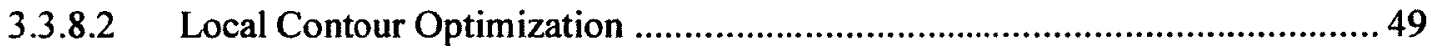

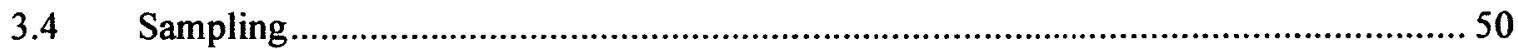

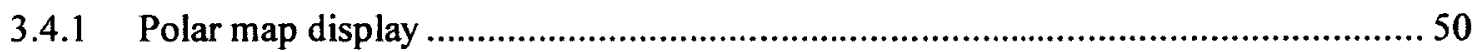

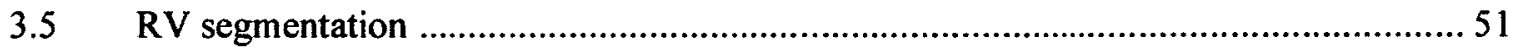

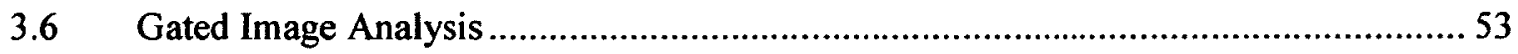

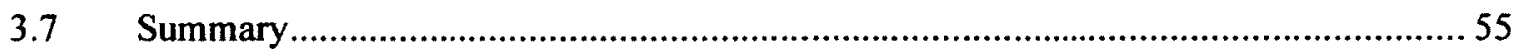

4 Chapter: Validation and Characterization ......................................................... 56

4.1 Appropriateness of the Model (Manual fitting of control points) .............................. 56

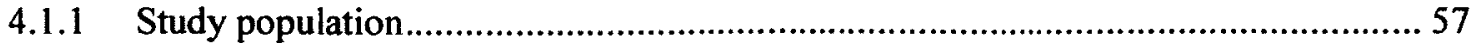

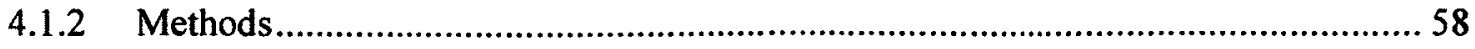

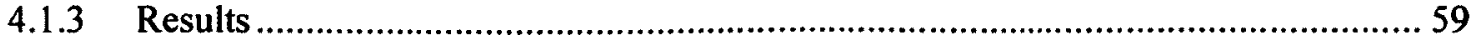




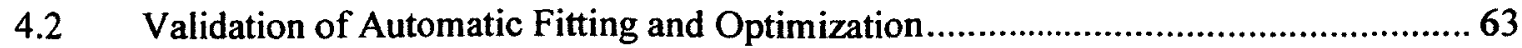

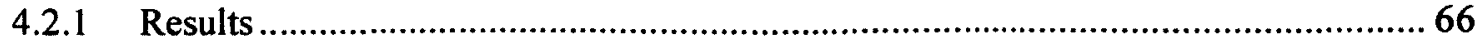

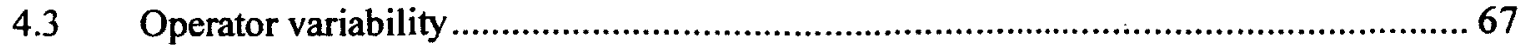

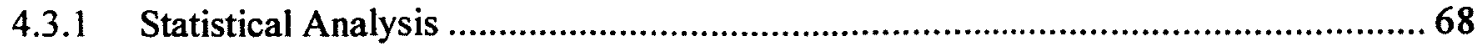

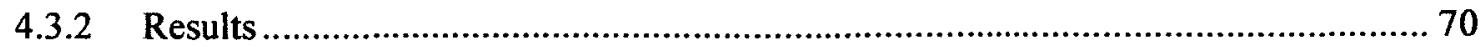

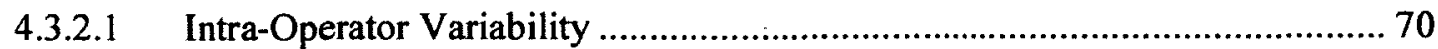

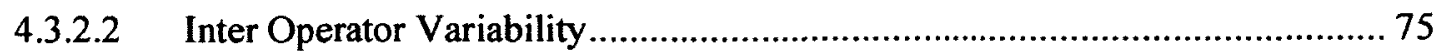

4.4 Validation of Cavity Volumes and Ejection Fractions ........................................ 77

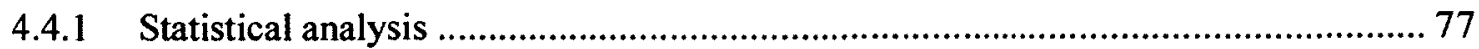

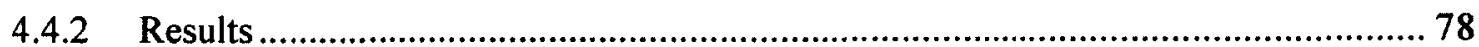

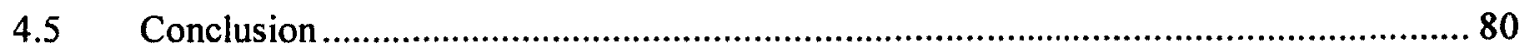

5 Chapter: Discussion ..................................................................................... 81

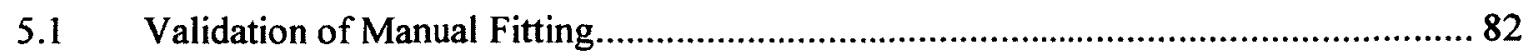

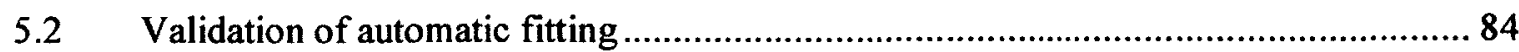

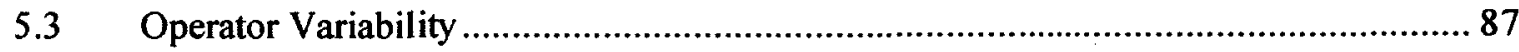

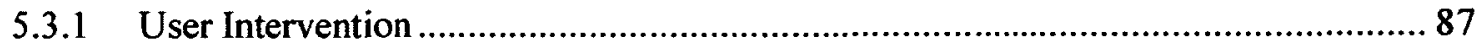

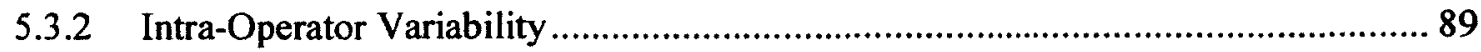

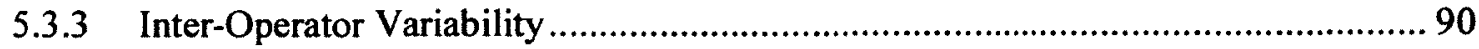

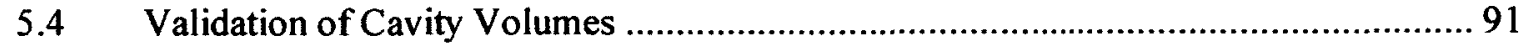

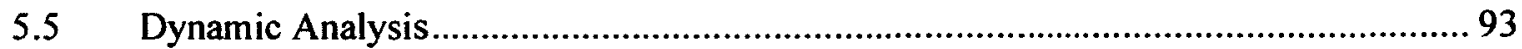

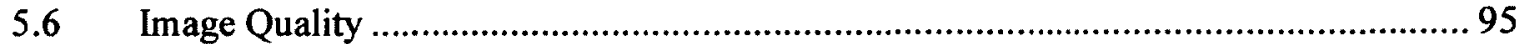

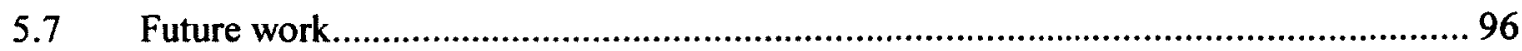

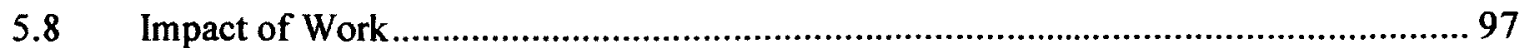

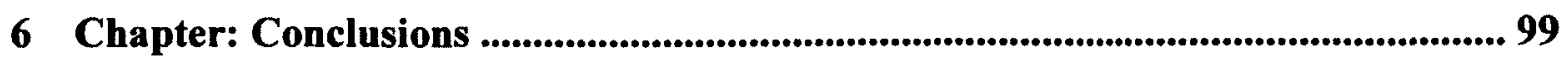

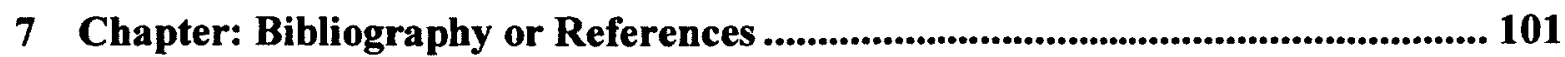




\section{List of Tables}

Table 2-1 : Comparison between polar map sampling techniques for the LV apical region (reproduced from [3]) 22

Table 3-1: Control Points and their degrees of freedom and initial location relative to the

LV Long Axis

Table 4-1: Population characteristics for subjects used in validation. 58

Table 4-2 - Summary of results per population category for manual fitting $(\mathrm{N}=20) \ldots . . .59$

Table 4-3 - Sample results from validation of manually fitted results $(\checkmark=$ pass,$x=$ fail $) .60$

Table 4-4: Results from adjustment of intersection control points

Table 4-5: Modified Control Points and their degrees of freedom and initial location relative to the LV Long Axis. 63

Table 4-6: Sample results from validation of automatically fitted results ( $\checkmark=$ pass, $\mathbf{x}=$ fail)

Table 4-7 : Validation Results for automatic fitting for 3 regions of the RV( $\checkmark=$ pass

$, x=$ fail $)$

Table 4-8 : Population characteristics for subjects for operator variability analysis 68

Table 4-9 : Number of interventions during operator variability testing. 70

Table 4-10: Mean \pm SD of cavity volumes and EF measurements acquired from PET and CMR cardiac images. 79

Table 5-1: Comparison of Pearson correlation coefficients $\left(r^{2}\right)$ and RPC (\%) values between current work and work by Klein et al [23]. 82

Table 5-2: Comparison of Cavity volumes and EF between current research and results from research by Grapsa et al for PH patients [22]. .93 


\section{List of Illustrations}

Figure 2-1: Schematic view of the human Systematic and pulmonary circulatory systems

Figure 2-2 : An illustration of a normal and a hypertrophic RV 6

Figure 2-3: Positron emission and annihilation with an electron (left) and cross section of a chest surrounded by a ring photon detector in PET (right). Also shown is the line of response (LOR) derived from detecting coincident events............................................ 8

Figure 2-4 : Rest-Stress Imaging protocol using Rubidium-82 and pharmacologic stress induced using Dipyridamole

Figure 2-5 : Demonstration pattern mismatch indicating region of decreased flow (ischemic) on the left and increased metabolism on the right.

Figure 2-6: A. Reorientation of images from camera reference frame to LV reference

frame. B. Partial ellipse fitting in different orthogonal planes during automatic

reorientation and basal short axis slice (bottom right) for verification

Figure 2-7 : Cardiac PET image views produced after reorientation [6]. (SA - short axis, HLA - horizontal long axis, VLA -vertical long axis) 16

Figure 2-8 : A simple illustration of a spline curve indicating control points, used to adjust the shape of the curve, and interpolation curve.

Figure 2-9 : Short axis (conical (1-8) and planar (9-24)), vertical long axis (VLA) and horizontal long axis (HLA) slices after automatic LV registration. Spline points are shown as circles and the model as red lines. The figure also includes results of RV registration to be discussed in chapter 3 . 
Figure 2-10 : Example tracer distribution as it transverses from the blood to myocardium (a) and corresponding blood (red) and myocardium (blue) time-activity curves (TACs) (b).

Figure 2-11: Cylindrical polar map sampling to create a hemi polar. Direction of the view of the polar map (base to apex) (Left). A sample slice divided into equal sectors (top right). A hemi polar created from cylindrical sampling (both right). FW - free wall ...... 23 Figure 3-1: RV quantification process dependency diagram. Faded steps were previous work. 28

Figure 3-2: A. Separation of the planar and conical region of the LV. B. Example of cylindrical coordinate representation of a sample point in planar region of the LV. C. Example of spherical coordinate representation of a sample point in the conical region of the LV. 32

Figure 3-3 : 3D Representation of the RV and its curved long axis for both normal and hypertrophic cases, depicting derivation of pseudo-cylindrical coordinate system. B. The polar coordinate in a single z-plane. 34

Figure 3-4 : Diagram of the RV spline model (green) relative to the previously described LV spline modell (red). Short axis slice (A) and horizontal long axis view of the model in a normal (B) and hypertrophic (C) heart. The control points are shown as black dots and described in Table 2.

Figure 3-5 (A. and C) : Illustration of the RV long axis (RVLA) in normal and hypertrophic hearts. (B and D) Morphed RV with a RVLA along the $\mathrm{z}$-axis after translating control points from LVLA to RVLA for both normal and hypertrophic cases. 
Figure 3-6 : RV ROI 3D volume generated from grid data output before sampling (A) Spline fitting model for short axis (SA) and horizontal long axis HLA .(B) and (C) RV ROI is bound by blue contours. D. RV hemi polar map alongside LV polar map .......... 41 Figure 3-7 : GUI display for SA slices, HLA (top right) and VLA (bottom left) showing $\mathrm{RV}$ contours after registration

Figure 3-8:RV hemi-polar map and LV polar map display (top left) and different views of 3D display of the RV and LV meshes

Figure 3-10 : Deducing the bounds for extent control point. A. Extent control point in normal and hypertrophic cases. B. Range of the extent control point.

Figure 3-11 : Sample slice RV slice showing fine tuning search window, boundaries of the search

Figure 3-12 : Cylindrical polar map sampling to create a hemi polar. Direction of the view of the polar map (base to apex) (Left). A sample slice divided into equal sectors (top right). A hemi polar created from cylindrical sampling (both right). FW - free wall51 Figure 3-13: Three vascular segments of the RV hemi polar. 52 Figure 3-14: Gated analysis report showing plot of RV Cavity volumes and EF for a hypertrophic RV along with RV and LV 3D meshes and polar maps of beating heart... 54 Figure 4-1: Reference diagram for control points as used in the validation 61 Figure 4-2: Hemi polar map of mean distances between sampling points in the paired datasets from for operator 1 and operator 2 . 71 Figure 4-3 : Histogram of means Euclidian distances for intra operator variability for operator 1 and operator 2 . 
Figure 4-4 : Linear Regression plot and Bland Altman plot depicting results from intraoperator variability for operator 1 and operator 2 .

Figure 4-5 : Hemi polar showing mean Euclidian distance from inter operator variability

Figure 4-6 : Histogram of mean Euclidian distance (errors) for 3 vascular territories of the $\mathrm{RV}$ region on interest. 76

Figure 4-7 : Linear Regression plot and Bland Altman plot depicting results for inter operator variability between operator 1 and operator 2 .

Figure 4-8 : Regression and Bland-Altman plot comparing cavity volumes and EF measurements acquired from PET and CMR cardiac images.

Figure 5-1 : A case of failed registration due to poor RV image intensity.(A) midsection

SA slice (B) HLA slice 83

Figure 5-2 : HLA slice indicating a case interference of the fitting algorithm by spillover from the RV. 86

Figure 5-3 : HLA slices showing variation of the location of extent control point 87 Figure 5-4 : SA slices shows 2 cases of indistinguishable shape of the RV towards the atrium due to the base of the pulmonary trunk. 88 Figure 5-5 : SA slices shows 3 cases indicating diminishing image intensity of RV ROI toward the atrium. 92 Figure 5-6 : A sample Kinetic modeling integrated report for the LV and RV. Uptake polar maps for both RV and LV are shown at the top half of the figure and time activity curves are shown at the bottom of the curve. 
Figure 5-7 : Vertical Long Axis (VLA) view of LV and Horizontal Long Axis (HLA)

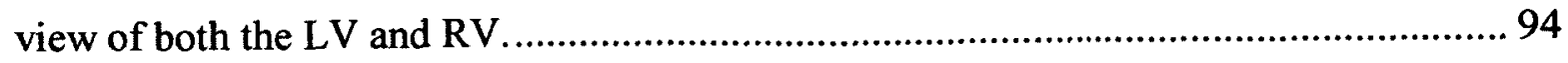




\section{List of Abbreviations}

RV - Right Ventricle

LV - Left Ventricle

RVLA - RV Long Axis

LVLA - LV Long Axis

ROI - Region of Interest

SA - Short Axis

LA - Long Axis

VLA - Vertical Long Axis

HLA - Horizontal Long Axis

TAC - Time Activity Curve

PM - Polar Map

EF-Ejection Fraction

EDV - End Diastole Volume

ESV - End Systole Volume

FWHM - Full Width at Half Maximum

CMR - Cardiovascular magnetic resonance imaging

FDG - 2-deoxy-2-( $\left.{ }^{18} \mathrm{~F}\right)$ fluoro-D-glucose

FTHA - $\left[{ }^{18}\right.$ F $]$ fluoro-6-thia-heptadecanoic acid 


\section{Chapter: Introduction}

\subsection{Problem Description}

Pulmonary Hypertension (PH) is a life threatening health condition in which pulmonary arteries (arteries of the lung) experience abnormal high blood pressure. The estimated average life expectancy of patients diagnosed with $\mathrm{PH}$ is about 3 years. $\mathrm{PH}$ is not well understood and effective therapies are lacking. Quantification of RV cardiac and metabolic function can provide understanding of risk factors associated with $\mathrm{PH}$ and how the disease progresses, and may aid in the development of therapies. The need for RV function analysis tools has been left largely unanswered. Specifically, an ongoing study at Ottawa Heart Institute on Right Ventricular Substrate Metabolism as a Predictor of Right Heart Failure in Patients with Pulmonary Arterial Hypertension can directly benefit from such functional analysis tools.

Left ventricular function estimation, however, is well established, and the University of Ottawa Heart Institute has developed a dedicated software package called FlowQuant ${ }^{\oplus}$ for $\mathrm{LV}$ functional analysis. This project appends RV analysis capabilities to the FlowQuant ${ }^{\mathcal{O}}$ package, leveraging on existing LV analysis resources. While some components of $\mathrm{LV}$ analysis can be applied for RV analysis, RV registration, defining the region of interest (ROI) from cardiac PET images, is a major challenge that has not been sufficiently addressed in the literature. The challenge can be attributed to the fact that the RV exhibits a wide range of anatomic shapes particularly in hypertrophic RV which increases the complexity of the registration algorithm. Despite this challenge it is evident that the need for an RV registration tool, that is preferably automatic, remains crucial in development of RV functional analysis tools. 


\subsection{Objectives}

The objective of this thesis is to develop an automated image analysis tool with optional operator intervention for defining the RV region of interest in 3D cardiac PET images.

This tool is intended for:

1) Quantification of RV cardiac function - the evaluation of efficiency of the heart as a pump by measuring cavity volumes and ejection fraction (EF) over the cardiac contractile cycle.

2) Quantification of RV molecular function - assessing the physiologic interaction with molecules of interest such as glucose and fatty acids with the RV muscle tissue.

Evaluation of RV function can help understanding the risk factors and causes of $\mathrm{PH}$, understand its progression as well as help develop effective therapies.

The second chapter provides background information on cardiac nuclear imaging. Emphasis is given to cardiac PET imaging because it is the modality of choice in this research. The second chapter also provides a summary of previous work relating to RV function analysis.

The third chapter provides in-depth details of the main contribution of this research; spline model for RV registration. The spline model is introduced and detailed descriptions of its implementation are systematically addressed as the chapter progresses. This chapter also introduces gated analysis, an application of the spline model to define RV ROIs when evaluating cavity volumes of the beating heart. 
The fourth chapter validates the spline models ability to accommodate a wide range of morphologies and characterizes its performance. A significant portion of this chapter characterizes the operator dependent variability of the model and the sampled image intensities. The chapter concludes with a validation of PET derived RV volumes and ejection fractions vs. cardiac magnetic resonance imaging (CMR) measured values in an early PH patient population.

Chapter 5 discusses these results in detail and contextualizes them with regards to current literature. The discussion highlights factors that may have influenced the results either negatively or positively. Future work and possible improvements to the model are also highlighted in this chapter.

The concluding chapter summarizes this work.

\subsection{Contributions}

This research resulted in functional semi-automated image analysis software that enables to register and sample the RV region of interest in cardiac PET and SPECT images, allowing accurate quantification of RV function cardiac function. This work also serves as a basis on which quantification of cardiac molecular function can be developed. This work supports ongoing research on Right Ventricular Substrate Metabolism as a Predictor of Right Heart Failure in Patients with Pulmonary Arterial Hypertension (PI: Lisa Mielniczuk) as the tool can be used to analyze glucose metabolism.

This research resulted in the following presentations:

- S Takobana, A Adler, L Mielniczuk, R Beanlands, RA deKemp, R Klein A Spline Model for Sampling of the Right Ventricle Myocardium from Cardiac PET Images, University of Ottawa Heart Institute Research Day, UOHI, May 2012.

- S Takobana, A Adler, L Mielniczuk, R Beanlands, RA deKemp, R Klein A Spline Model for Sampling of the Right Ventricle Myocardium 
from Cardiac PET Images, Molecular Function and Imaging Symposium, UOHI ,June 2012.

- S Takobana, Automatic Quantification of Right Ventricular Function as a Diagnostic Aid for Pulmonary Hypertension, Biomedical Engineering Seminar (BIOM 5800), Carleton University, Ottawa, February 2012. 


\section{Chapter: Background}

The human heart is a four chambered organ forming the core of the circulatory system. Its main role is to propel blood through the pulmonary and systematic circulatory loops. The chambers of the heart are namely, the right and left ventricles and the right and left atria. Figure 2-1 below depicts the anatomy of the heart, with the chambers labeled.

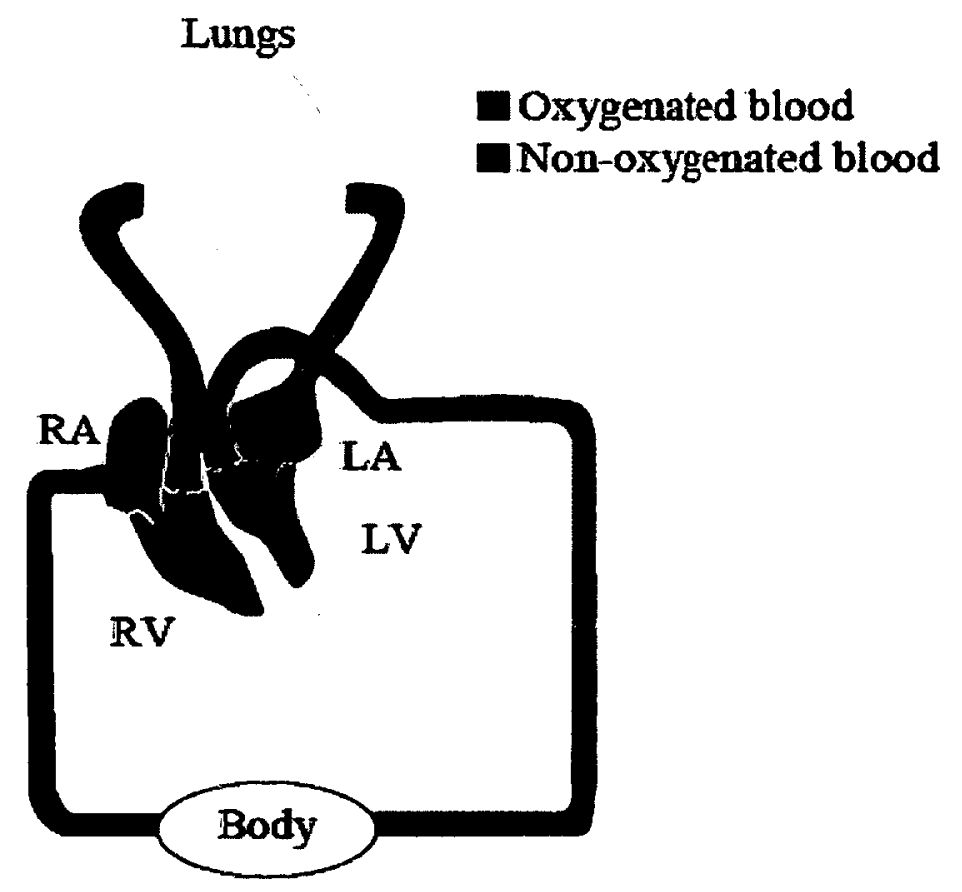

Figure 2-1: Schematic view of the human Systematic and pulmonary circulatory systems The circulatory system comprises of the systematic circulation and pulmonary circulation. Systematic circulation is powered by the left ventricle (LV), which has the strongest muscle of all the heart chambers, and propels blood to and from the body. The pulmonary system, is powered by the right ventricle, and sends deoxygenated blood to the lungs for re-oxygenation and returns it to the left heart to be pumped into the rest of the body via the systematic circulation. 
Pulmonary hypertension $(\mathrm{PH})$ is a disease in which the blood pressure in the pulmonary arteries is abnormally elevated and left untreated can lead to RV (right ventricle) hypertrophy (enlargement) and dysfunction (malfunction) [2]. There are several causes of pulmonary hypertrophy which can be summarized into broad categories including pulmonary arterial hypertension, left heart diseases, lung diseases or hypoxemia (low of oxygen in arterial blood) [2]. Hypertrophy, enlargement of the right ventricle, occurs when the heart, in this particular case the RV, remodels in an attempt to keep up with the pressure demand of pulmonary arteries and maintain stroke volume $[2$, 4]. Subsequently hypertrophy and increased pressure leads to dysfunction characterized by decreased cardiac output and poor ventricular contractibility [4].

Compared to the LV, which is a muscular ellipsoid; the RV is crescent shaped giving it a lower volume-to-surface ratio [19]. When the RV becomes hypertrophic it resorts to a more concentric shape and the interventricular septum flattens $[4,19]$. In hypertrophic cases as shown in Figure 2-2, the RV becomes more spherical and develops an increased cross sectional area that can surpass that of the LV.

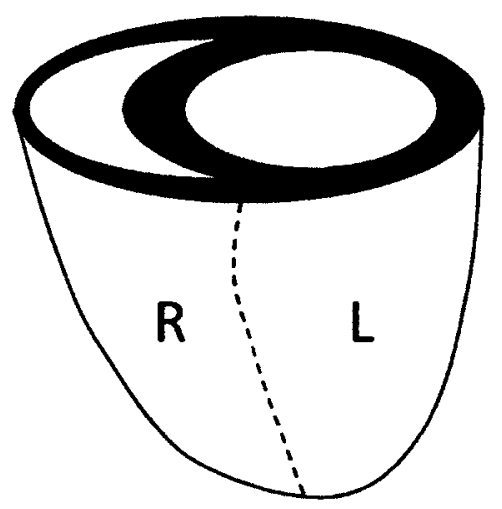

Norm

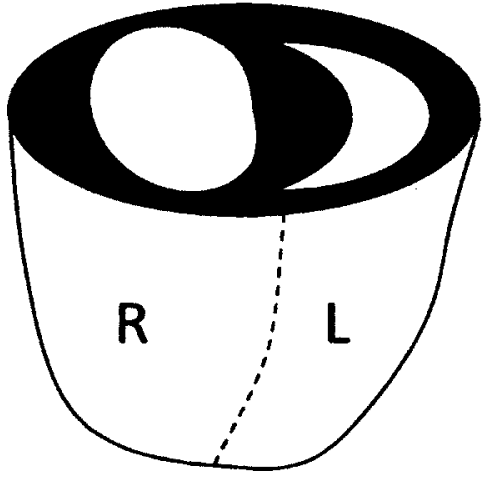

Hvpertrophic

Figure 2-2 : An illustration of a normal and a hypertrophic RV. The hypertrophic RV (right) has a thicker wall and cavity size than normal RV (left). 
RV hypertrophy can easily be identified by visual interpretation of ultrasound imaging, while evaluation of the RV functionality is improving with the advancement of imaging modalities such as positron emission tomography (PET) and single photon emission tomography (SPECT). While ultrasound is inexpensive, does not expose the patient to radiation, and, and is less time consuming than PET and SPECT, it is limited by the inability to measure functional physiologic information such as perfusion and rate of metabolism [17].

SPECT and PET are both nuclear technologies that employ molecules labeled with radionuclides in trace amounts to non-invasively image the internal molecule distribution using an external detector. Both modalities can provide gross information about anatomic structure [17].

\subsection{Positron Emission Tomography (PET) and Single Photon Emission Tomography (SPECT)}

PET can be used to measure myocardial blood flow (MBF) and metabolism using physiological substrates prepared from positron emitting isotopes with short half-lives such as carbon-11, fluorine-18, oxygen-15 and nitrogen-18 $[6,17]$. These substrates are injected in small quantities so as to not invoke a physiologic response and are therefore commonly referred to as tracers.

Positrons, positively charged particles with same rest mass as electrons, are emitted from parent nucleus and annihilate with electrons in the surrounding medium, emitting a pair of photons at almost exactly $180^{\circ}$ separation [17]. (Figure 2-3) Coincidence detection in PET allows detectors to register photon pairs hitting opposite 
detectors nearly simultaneously (Figure 2-3). Millions of coincident events are recorded all around the patient providing the projection of activity distribution. The measured activity distribution is reconstructed into stacks of cross-sectional images, called tomographs, forming a three-dimensional volume image [17]. PET can be integrated with $\mathrm{x}$-ray CT enabling PET/CT scans which give complementary anatomic and physiologic data in a single session. In cardiac imaging, this PET-CT arrangement insures that the heart is accurately positioned within the PET field of view (FOV) and corresponding images are spatially aligned [17]. The CT data is also used to accurately correct PET images for photon attenuation.
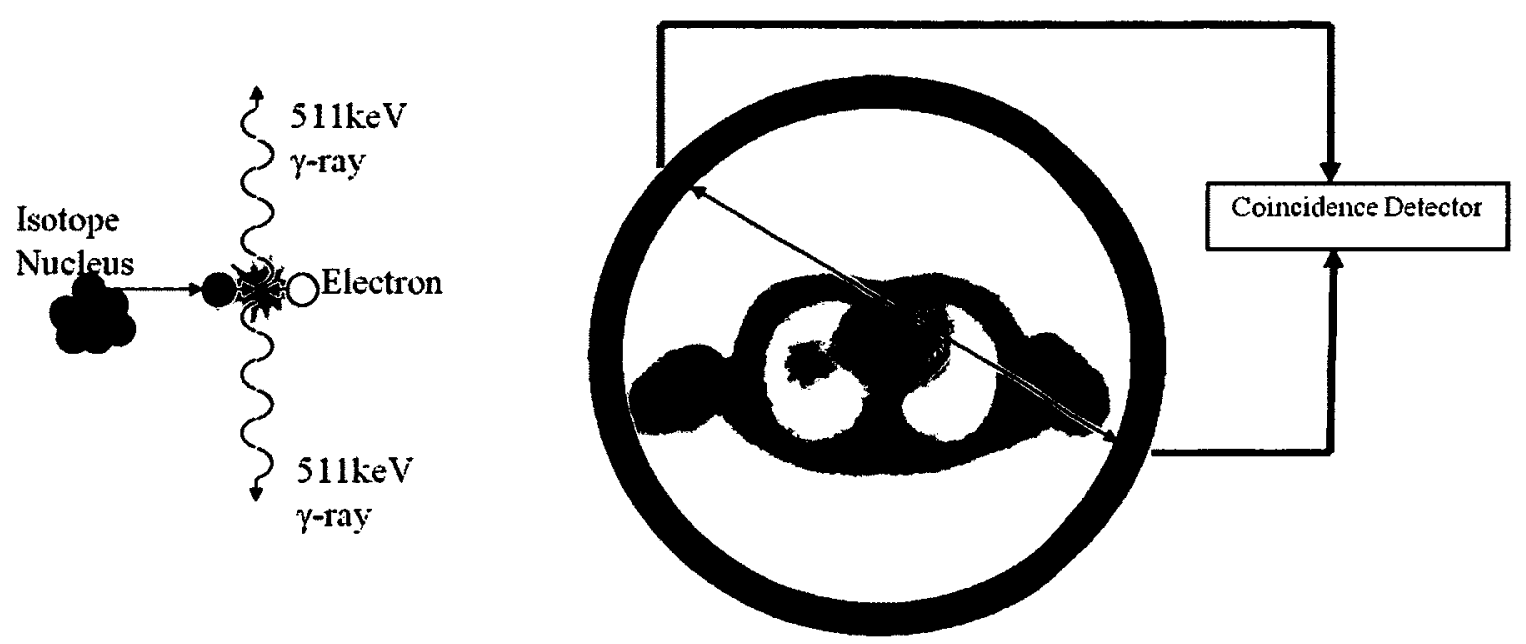

Figure 2-3 : Positron emission and annihilation with an electron (left) and cross section of a chest surrounded by a ring photon detector in PET (right). Also shown is the line of response (LOR) derived from detecting coincident events.

Unlike PET, SPECT uses single photo-emitting radiotracers. As in PET, the radio-isotopes localize according to the metabolic properties of the molecule that they are bond to. The radio-isotope emit gamma rays which are converted to visible light using a crystal scintillator on the external detector $[2,17]$. Collimators, which are coupled to the 
scintillator allow only photons at specific directions to reach the detector, while blocking all other photons. During the image acquisition, the detector heads are rotated around the patient, capturing multiple projections of the image [17]. The projections may be used to reconstruct tomographs of the imaged volume. With suitable radio-tracers, SPECT is commonly used in cardiology to image myocardial perfusion.

Both PET and SPECT have capabilities of capturing static and cardiac gated uptake images. In static image acquisition the image is obtained when the tracer uptake has reached a plateau and tracer redistribution process has stabilized. Static uptake images are used to estimate the net overall tracer uptake.

Electrocardiogram (ECG) gated images are acquired by binning each photon into a bin corresponding to a phase of the cardiac cycle. The projections in each bin are reconstructed into individual images corresponding to the phase of the cardiac cycle, thus the reconstructed images are of the beating heart and can be used to measure cavity volumes and ejection fractions. The images are acquired over several minutes and are thus an average of hundreds of heart beats, not a single contraction. Respiratory gating information is sometimes also acquired and may be used for respiratory motion correction.

In dynamic imaging, image slices are obtained by serial temporal sampling at contiguous time intervals starting immediately after the tracer injection. Dynamic imaging allows one to measure tracer distribution over time. Dynamic imaging is challenging with typical rotating head SPECT cameras, and is more commonly used with PET. 


\subsection{Comparison between PET and SPECT}

Although SPECT and PET ascribe to similar basic principles SPECT has lower spatial resolution than PET. SPECT uses a collimator in front of the detector which tends to reduce photon sensitivity, whereas no physical collimation is used in PET. PET has roughly 2 orders of magnitude greater sensitivity then SPECT, which can be leveraged to improve its temporal resolution and/or reduce the amount of radiation used [2]. PET can be more easily and accurately corrected for attenuation compared to SPECT [17]. SPECT imaging technology, however, is still less expensive than PET. Furthermore, a wide range of SPECT tracers are commercially available at a fraction of the cost of most PET tracers due to longer tracer half-lives, centralized isotope production, and an established distribution network $[2,3,17]$. Thus, while PET is a preferred imaging technology, SPECT is more commonly used clinically.

This work was conducted within the larger scope of probing disease mechanisms and therapy approaches for pulmonary hypertension using small animal models and clinical data using PET. While the following chapters primarily describe processing of PET images, the methods are expected to be easily transferable to SPECT imaging with little or no adaptation. To demonstrate the utility of cardiac PET, two common examinations are highlighted:

\subsubsection{Perfusion Imaging with PET}

Cardiac images are acquired in cardiac rest phase followed by cardiac stress phase. A CT scan is performed for patient alignment and attenuation correction. The patient is then injected with a perfusion tracer (e.g. ${ }^{13} \mathrm{~N}$-Ammonia, or ${ }^{82} \mathrm{Rb}$ ) through an intravenous line, followed by a PET acquisition ( $<10$ minutes) in a normal resting 
state.[6] Once the rest scan is completed, a cardiac stress state is induced in the patient by administering a pharmacologic drug or through physical exercise and the tracer injection and PET acquisition similar to the rest phase is repeated $[6,20]$. A second CT acquisition may follow to correct the stress scan for attenuation [6]. With some stress agents, an antidote may be administered part way through the PET acquisition to help the patient return to normal cardiac state and reduce side effects $[6,20]$. Figure $2-4$ outlines an example ${ }^{82} \mathrm{Rb}$ rest-stress protocol in PET cardiac imaging using Dipyridamole as a stress agent and aminophylline as an antidote.

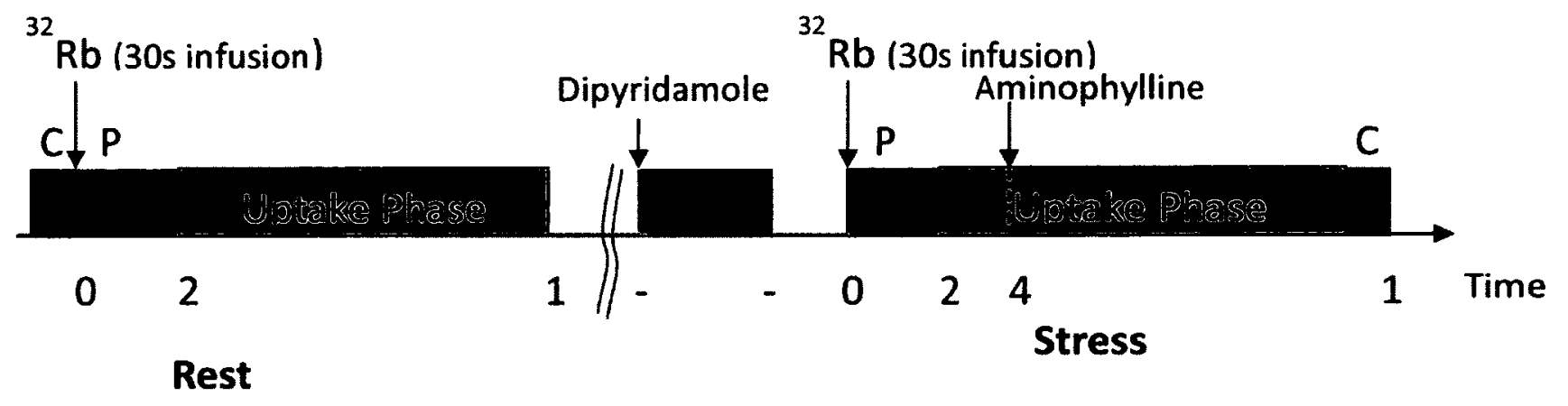

Figure 2-4 : Rest-Stress Imaging protocol using Rubidium-82 and pharmacologic stress induced using Dipyridamole

The rest and stress scans are interpreted together to locate partial ischemia (present at stress and less at rest), or complete occlusion (reduced tracer uptake at rest and stress). Gated images may also be reconstructed and interpreted for wall motion abnormalities, and reduced cardiac output. 


\subsubsection{Viability Imaging}

Viability imaging provides insight to the degree of healthiness of the myocardium (heart muscle). Nuclear imaging modalities provide the ability to categorize myocardium as viable, necrotic (dead due to injury), normal, or hibernating. Viability imaging is critical in research and clinic because it enables to monitor changes in cardiac function during therapy, revascularization, and disease progression [21, 43]. Viability imaging has also been shown to be effective in guiding therapy to improve outcomes [21, $43,44]$. Because of its high sensitivity and specificity PET imaging remains superior in viability detection using perfusion-metabolism match pattern scheme [21]. In normal myocardium, both perfusion and viability tracer uptake are present, but in necrotic or scarred tissue viability tracer uptake is reduced [21]. Due to lower metabolic activity even if blood supply is normal [43].

Cases with perfusion-metabolism pattern mismatch, where perfusion is decreased but metabolism remains high, as demonstrated in Figure 2-5, indicate that the tissue is viable but may be ischemic or hibernating [21]. In tissue that is ischemic (oxygen deprived) myocytes derive their energy from glycogen through anaerobic (without oxygen) glycolysis (breakdown of glucose to energy) [21, 22, 31], rather than their preferred fuel sources, fatty acids. 


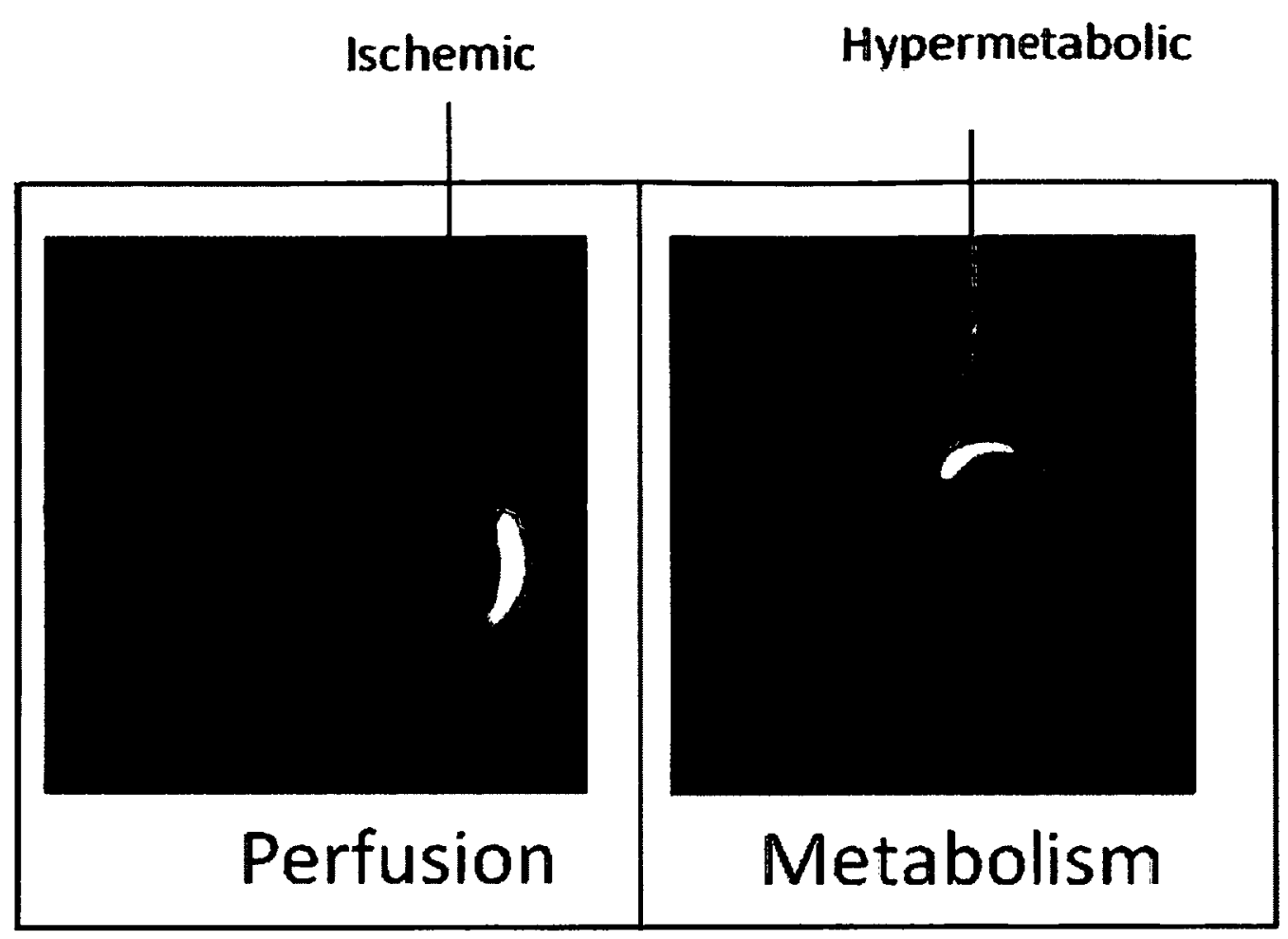

Figure 2-5 : Demonstration pattern mismatch indicating region of decreased flow (ischemic) on the left and increased metabolism on the right.

Using 2-deoxy-2-( $\left.{ }^{18} \mathrm{~F}\right)$ fluoro-D-glucose( FDG) in PET, a glucose analog commonly used as viability tracer, it is possible to guide patient therapy in a manner that improves outcomes while reducing risks associated by inappropriate procedures $[31,33,43]$. Viability imaging can also be used to monitor improvements in regional wall movement and global ventricular ejection fraction particularly after revascularization [21].

\subsection{Image Processing}

Various cardiac function interpretation software packages exist, which have common functionality to aide in clinical interpretation of LV function. In this work we will focus on FlowQuant ${ }^{\circ}$, a nuclear image processing software developed at the 
University of Ottawa Heart Institute (OUHI). FlowQuant ${ }^{\mathbb{O}}$ performs static, gated, and dynamic image analysis primarily for the left ventricle, and caters to translational research from small rodents to humans. This research builds upon the existing analysis of the LV, and adds the ability to perform functional measurements for the right ventricle. A brief explanation of LV image analysis with FlowQuant ${ }^{\circledR}$ follows:

\subsection{Image Reorientation}

The reconstructed images are in a camera reference frame and roughly traverse the patient transaxially. Klein et al.'s work [6], on which this work was based, describes how the image volume is reoriented to a standard LV reference frame through iterative fitting of partial ellipses in three orthogonal planes as illustrated in Figure 2-6B [6]. The images are reoriented into the $\mathrm{LV}$ reference frame through a $3 \mathrm{D}$ rotation and translation as depicted in Figure 2-6A below. Briefly, a partial ellipse is fit to three orthogonal image planes (transverse, mid-vertical long axis (VLA), mid-horizontal long axis (HLA)) through the LV to resolve the location, angles, and approximate size of the LV. The reoriented image is a stack of short-axis (SA) slices comprising the image volume in a standardized LV reference frame. 


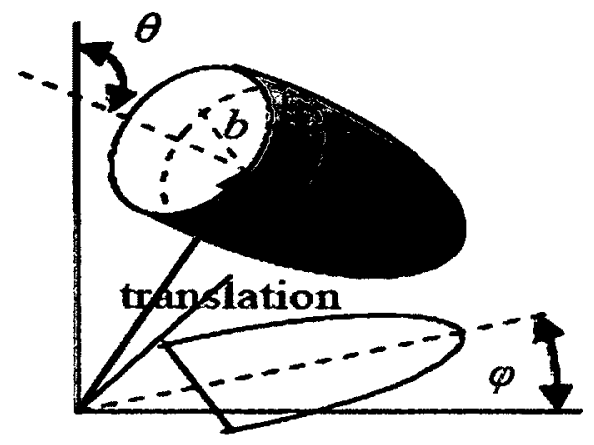

A.

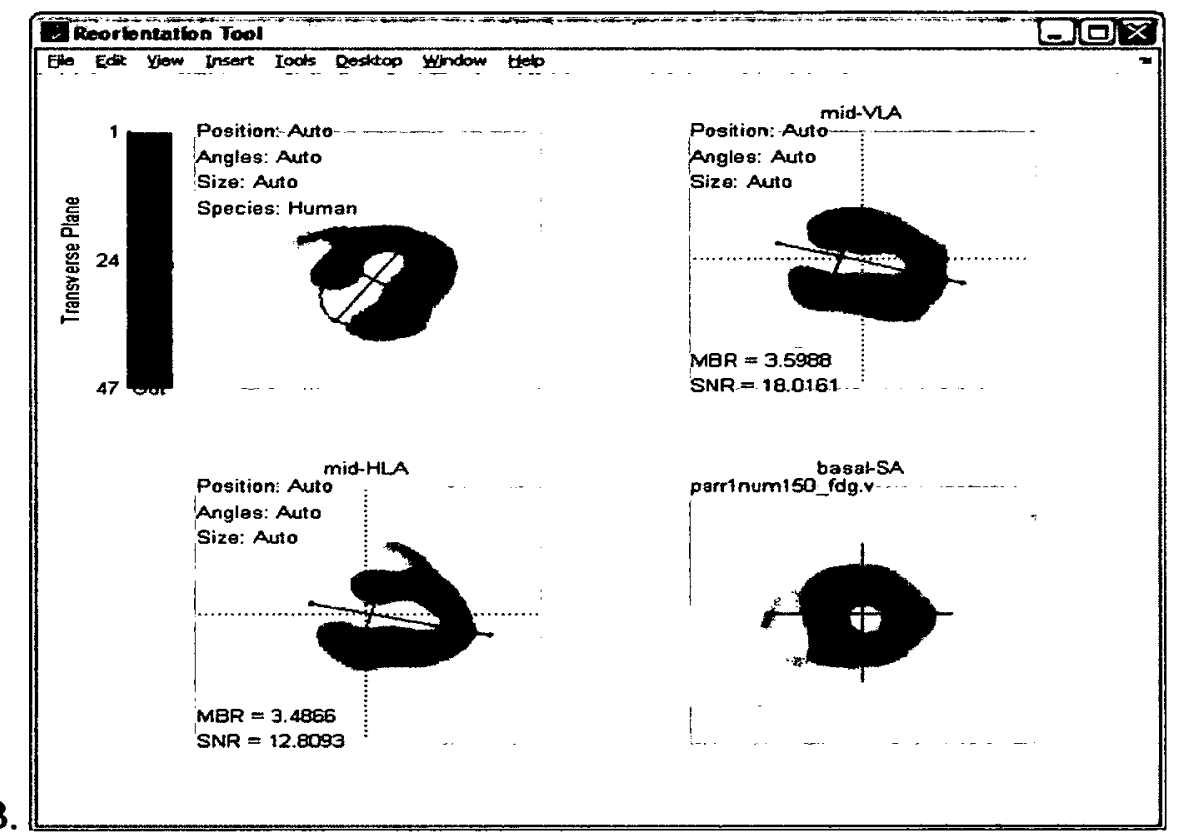

Figure 2-6: A. Reorientation of images from camera reference frame to $L V$ reference frame. B. Partial ellipse fitting in different orthogonal planes during automatic reorientation and basal short axis slice (bottom right) for verification

Since the RV is conjoined to the $\mathrm{LV}$ it is also reoriented to a standard reference frame as part of the LV reorientation process [6]. The reoriented views can be displayed as short axis (SA), horizontal long axis (HLA), and vertical long axis (VLA) as depicted in Figure 2-7. 


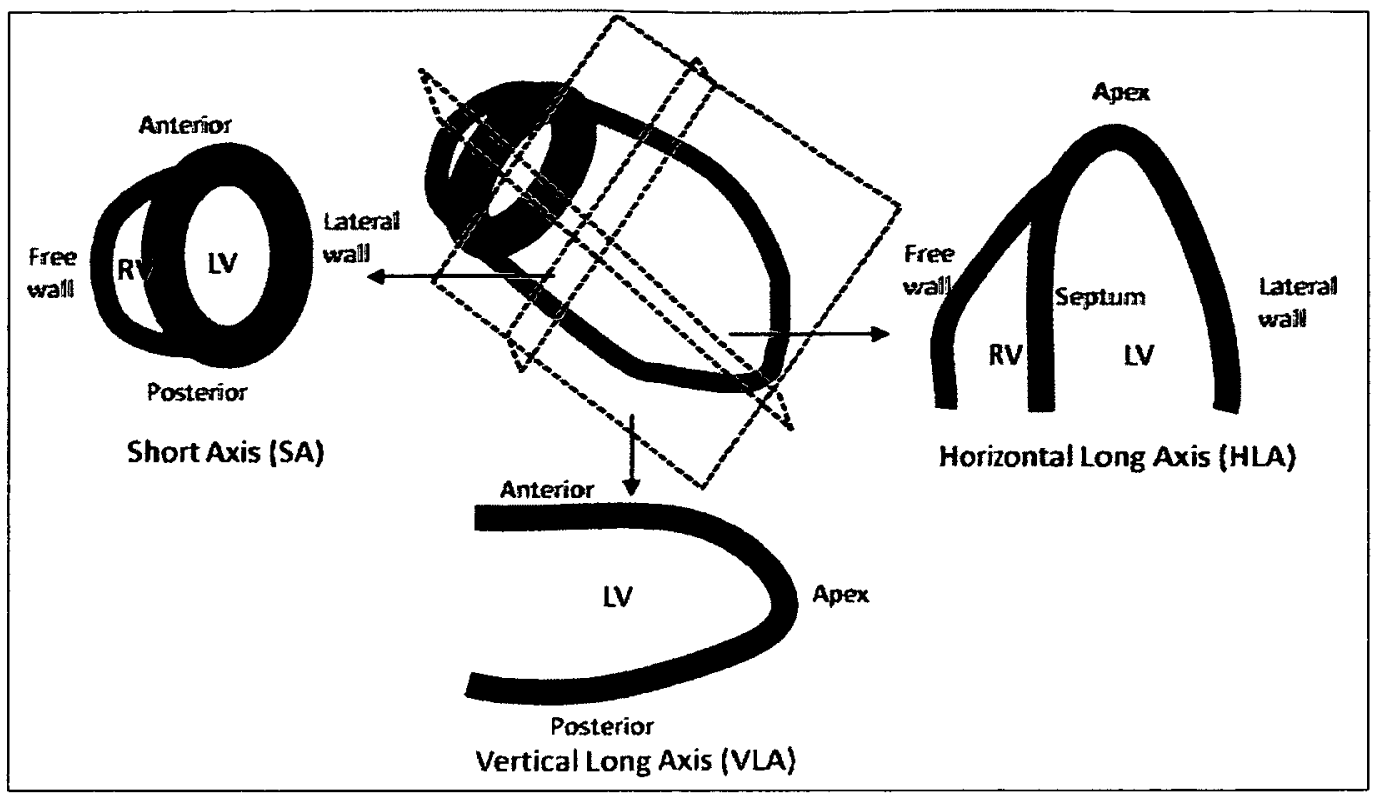

Figure 2-7 : Cardiac PET image views produced after reorientation [6]. (SA - short axis, HLA horizontal long axis, VLA -vertical long axis)

\subsection{Sampling and Registration}

\subsubsection{Splines}

Splines are mathematical functions expressed as piecewise polynomials that are used to interpolate a sequence of points called control points, to represent a shape of a surface or curve [7][60]. Spline generates smooth curves and the shape curve can be modified by adjusting positions of control points (Figure 2-8).

Deformable spline models are initialized with a rough estimate of the surface to be represented. The control points can then be adjusted to continuously deform the shape until it represents the actual data to some degree of accuracy [58]. 


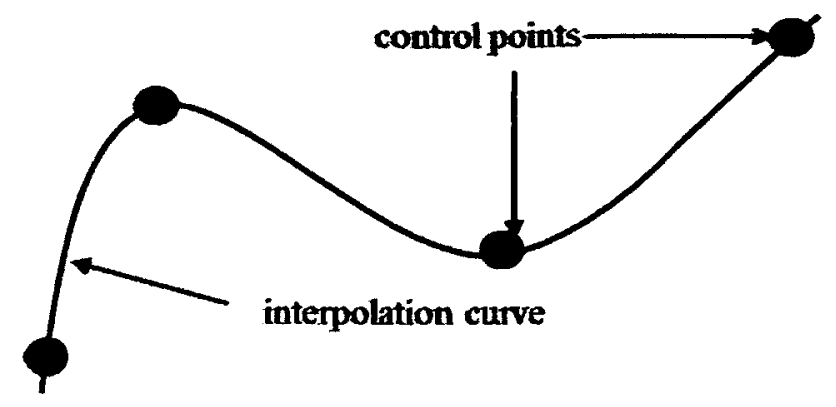

Figure 2-8 : A simple illustration of a spline curve indicating control points, used to adjust the shape of the curve, and one possible interpolated curve.

Alternative registration techniques using edge detection algorithms were regarded as suboptimal for this problem as they may be susceptible to failure in a case where there is discontinuity (e.g. myocardial defect) [7], or in the presence of other nearby organs with high tracer concentration (e.g. liver, stomach, lungs). A further advantage of the spline model is that the operator can easily interact with the spline model to correct the fit if need be. The spline algorithm used in this research (as well as for LV registration) is cubic-spline that is included in the basic MATLAB package and is based on the work of de Boor [61].

\subsubsection{Registration and Sampling}

Registration of LV myocardium by applying a deformable spline model defined by 13 sample points with 1 degrees of freedom each ( 4 radial points for short-axis slices through the LV cavity, LV base, and left atrium and one radial point for the apex extent) [6]. The $\mathrm{LV}$ region from the cavity to the atrium is represented in cylindrical coordinates, while the apical region is represented in spherical coordinates. This representation allows radial profiles that intersect myocardium almost orthogonal in both regions. During registration, the energy maximizing optimization algorithm optimizes 4 radii at $90^{\circ}$ 
increments at LV cavity, LV base and LV atrium slices as well as the control point at the RV apex [6].

The spline model sampled the image using 24 rings with 36 segments each (at $10^{\circ}$ intervals) centered on the LV long axis, which is centered on the reoriented image. Rings 1 through 8 encoded radii in a conical coordinate system (spanning at $10^{\circ}$ intervals from the long axis) and provided for radials that was roughly perpendicular to the apex surface $[6,23]$. The remaining 16 rings encoded radii in a cylindrical coordinate system and sampled the LV cavity through to the left atrium $[6,23]$. The combination of spherical and cylindrical sampling is often referred to as bottlebrush coordinate system. Figure 2-9 shows short axis, vertical long axis and horizontal long axis after bottlebrush registration. The LV contour is depicted by the red sampling points. Slices 1 through 8 correspond to flattened cones in the apex region.

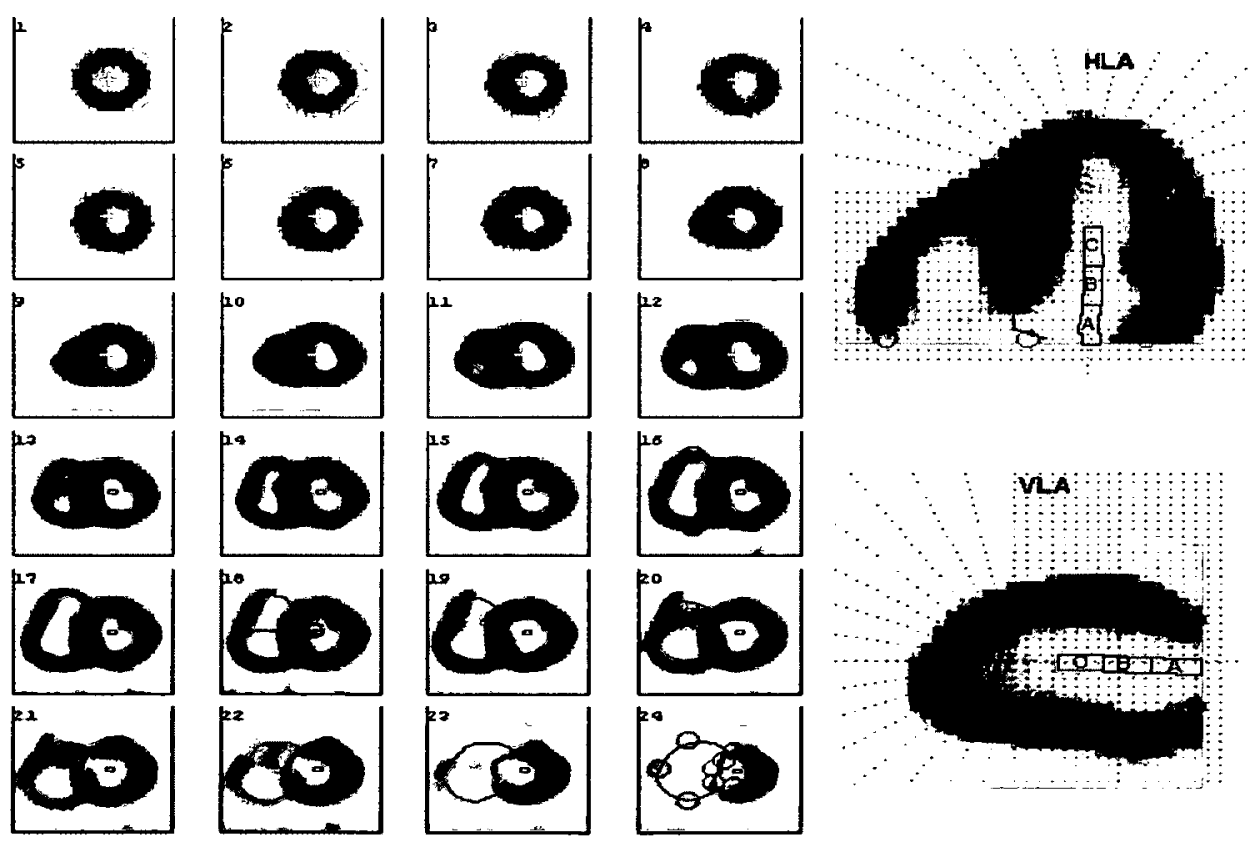

Figure 2-9 : Short axis (conical (1-8) and planar (9-24)), vertical long axis (VLA) and horizontal long axis (HLA) slices after automatic LV registration. Spline points are shown as circles and the model as red lines. The figure also includes results of RV registration to be discussed in chapter 3. 
It is desirable to minimize the number of control points and degrees of freedom to avoid overwhelming the operator and reduce computational complexity. However, too few degrees of freedom can reduce the ability of the model to accurately describe the spectrum of morphologies. The following chapter describes a similar spline model adjoined to the LV model for sampling the RV that follows similar principles.

\subsection{Analysis of dynamic PET images}

Dynamic analysis can be used to quantify physiologic functions such as myocardial blood flow (MBF) through changes in tracer concentration in the blood and myocardium over time. Sequences of images are acquired at contiguous time intervals and measure the tracer concentration (e.g. $\mathrm{Bq} / \mathrm{cc}, \mathrm{mol} / \mathrm{cc}$ ) as a function of time as blood transverses from left heart and arteries to the myocardium and the tracer bio-chemically interacts in the tissue $[6,23]$. Regions of interest (ROIs) placed on the myocardium can be sampled at different time intervals to generate myocardial time-activity-curves (TACs) as shown in Figure 2-10 as a blue line. Likewise, an ROI of the LV blood cavity can be sampled to produce an arterial blood TAC, red line in Figure 2-10. Compartment models can be used to model the kinetic behavior of the tracer. The model consists of transfer rate parameters between the blood (input) and myocardium (output) TACs $[6,17,23]$. These parameters are fit to the TACs data to generate regional maps of the tracer molecule rate of interaction with the tissue. Specific kinetic models are specific to the tracer molecule, tissue type, and imaging protocol, and are beyond the scope of this work. 


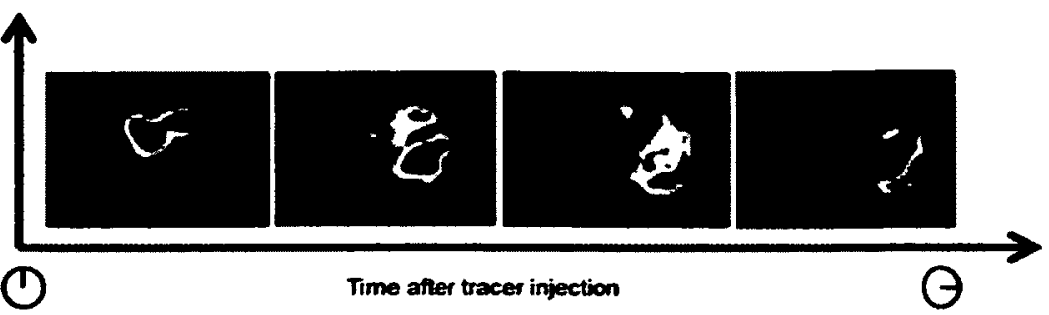

(a)

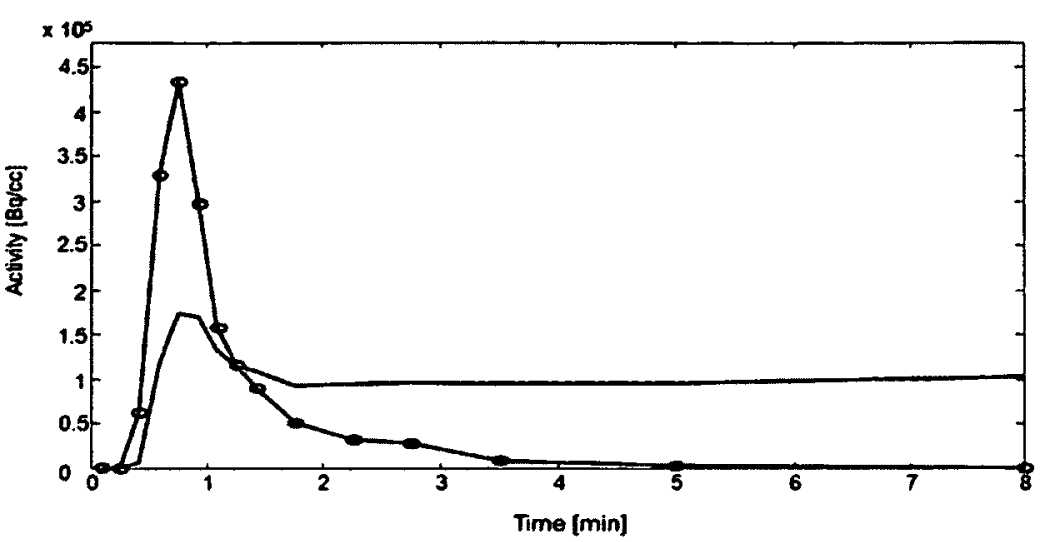

Figure 2-10 : Example tracer distribution as it transverses from the blood to myocardium (a) and corresponding blood (red) and myocardium (blue) time-activity curves (TACs) (b).

\subsection{Gated Image Analysis}

In gated image analysis a complete ECG cardiac cycle defined by $\mathrm{R}-\mathrm{R}$ interval is equally partitioned into gates of equal intervals [17]. During many cardiac cycles, projection data from each gate is acquired and stored in a separate bin corresponding to the phase of the cardiac cycle. After data acquisition each bin is individually reconstructed to generate an image corresponding to a specific phase in the cardiac cycle $[8,15,17,18]$. An LV model can be fit to these images and processed to calculate a volume curve representing cavity volumes for each cardiac phase. End systole (ES) and end diastole (ED) volumes mark the extremes of the cardiac contraction and are commonly reported along with the ejection fraction (EF) $[6,17,18]$. EF is an important 
marker of cardiac output, and is routinely used for prognostication [8]. Ejection fraction can be computed as follows;

$$
E F=\frac{E D V-E S V}{E D V} * 100 \%
$$

Where $E F=$ Ejection Fraction, $E D V=$ End Diastole Volume, $E S V=$ End Systole Volume

\subsection{Display}

Visualization of $3 \mathrm{D}$ volumes is commonly achieved using multiple slices through the image volume. However, $\mathrm{LV}$ polar maps are also used to visualize the 3D myocardium on a single 2D map, and have been standardized to map the apex region to the center of the map and the base at the radial extent $[3,5]$. Several techniques have been proposed to render LV polar maps, differing in the way the apical region (extending from the cavity to the apex) is sampled [3]. Cylindrical, spherical, and a combination of spherical and cylindrical coordinate systems have been explored. In all cases a long axis is defined between the apex and valve plane through the middle of the left ventricle cavity. While cylindrical or spherical coordinate systems are simple to implement, they are suboptimal for the typical LV morphology since a large fraction of the radials intersect the LV myocardium at oblique angles, making it difficult to precisely locate the mid myocardium and endo- and epi-cardial edges. Therefore a combined cylindricalspherical coordinate system (also referred to as a bottlebrush) is typically used. These coordinate systems are illustrated in Table 2-1. 
Table 2-1 : Comparison between polar map sampling techniques for the LV apical region (reproduced from [3])

\begin{tabular}{|c|c|c|c|}
\hline $\begin{array}{l}\text { Sampling } \\
\text { technique }\end{array}$ & Spherical & Radial & Cylindrical \\
\hline Apex & & & \\
\hline Polar maps & & & \\
\hline Description & $\begin{array}{l}\text { Samples the surface at } \\
\text { equal sectors each of } \\
\text { angle } \theta^{\circ}, \text { at } \varphi^{\circ} \text { from the } \\
\text { long axis. } \\
\text { Achieves uniform sampling } \\
\text { for spherical surfaces but } \\
\text { omits the epical region }\end{array}$ & $\begin{array}{l}\text { - Samples the surface at equal } \\
\text { sectors } \\
\text { - Achieves complete uniform } \\
\text { sampling for spherical } \\
\text { surfaces } \\
\text { - Ideal for the LV because of its } \\
\text { elliptical shape }\end{array}$ & $\begin{array}{l}\text { - Cross-sectional slices at } \\
\text { equal intervals } \\
\text { - Slices differ in size; smaller } \\
\text { towards apex } \\
\text { - Ideal for cylindrical } \\
\text { surfaces }\end{array}$ \\
\hline
\end{tabular}

The orientation of the polar map follows a convention agreed upon by the American Society of Nuclear Cardiology and American Heart Association (Dilsizian, et al., 2009). Regions in the polar maps can be mapped to corresponding major arteries using a standard database; however, this is of limited utility due to a natural variability in the populations' anatomy and variability due to medical intervention such as bypass surgery. While guidelines have not been issued for RV maps, the RV can be visualized with the free wall mapped to a hemi polar as shown in Figure 2-11 [5]. Chiba et al provided a comprehensive approach in SPECT cardiac data processing and visualization of the RV using cylindrical polar map sampling as shown in Figure 2-11. From the RV region of interest (ROI) they manually selected between 6 and 8 short axis slices, each slice partitioned into 30 sectors over 180 degrees, from base to apex of the RV [5]. Maximum counts per pixel for each sector were measured, and normalized, generating a 
circumferential profile [5]. A hemi polar is obtained by applying the profile to all the short axis slices and plotting the data in polar coordinates $[3,5]$. This work provides a generic insight for polar map sampling of $\mathrm{RV}$ ignoring the fact that slices tend to decrease in size towards the apex. Despite the mentioned challenge and because the technique is generic, it is reasonable to apply to hypertrophic cases because of their diverse morphologies.
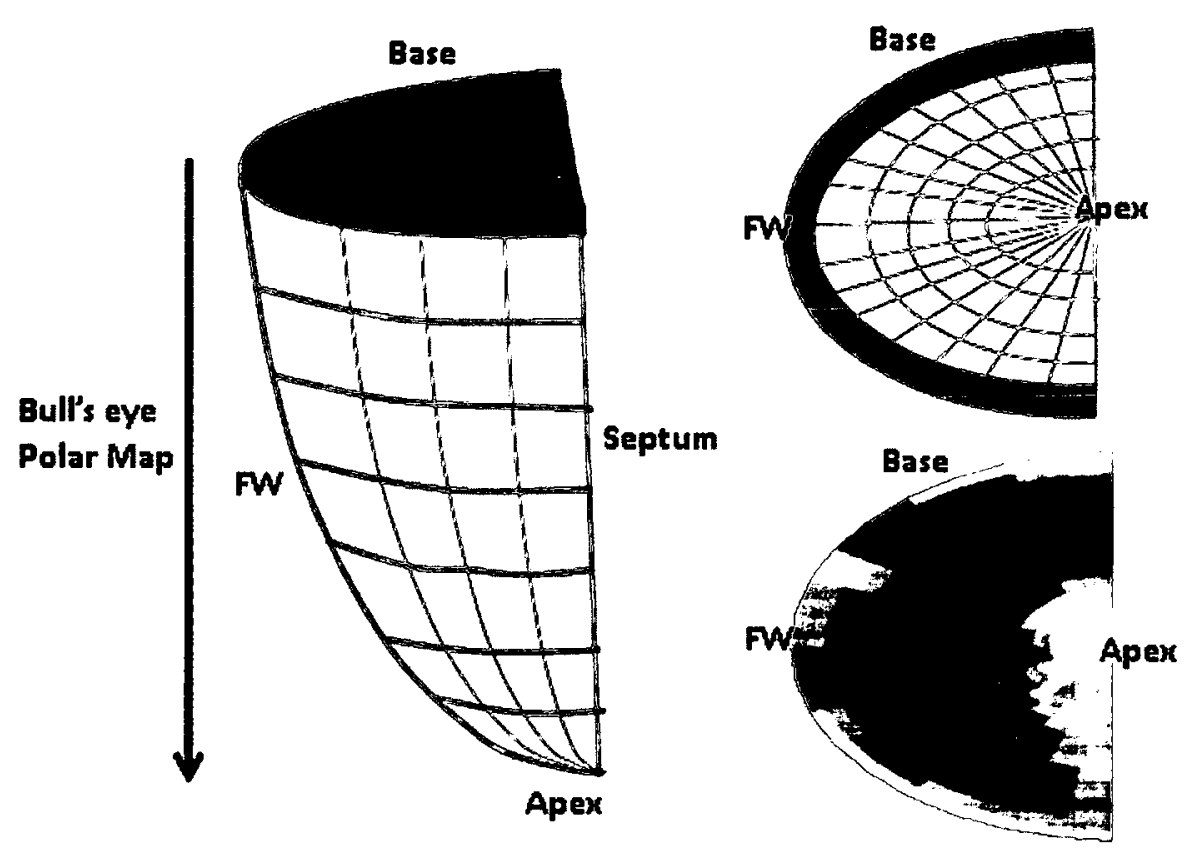

Figure 2-11: Cylindrical polar map sampling to create a hemi polar. Direction of the view of the polar map (base to apex) (Left). A sample slice divided into equal sectors (top right). A hemi polar created from cylindrical sampling (both right). FW - free wall 


\subsection{Other Work on Right Ventricular Imaging and Quantification}

A wide range of literature covers nuclear image data acquisition, processing and visualization exclusively for the left ventricle. It seems plausible that some of the techniques employed can be adapted for right ventricular studies.

There are a handful of nuclear imaging related studies that specifically explore the right ventricular perfusion and function. Most of the literature indicated that the use of SPECT as the imaging modality of choice which can be attributed to the state of imaging technology at the time, and prevalence of different imaging technologies. The rising popularity of PET and greater pool of available tracers promises a wide range of research and clinical applications.

Pereira et al assessed right ventricular perfusion and function using ${ }^{99 \mathrm{~m}} \mathrm{Tc}$ sestamibi (Technetium-99m isotope based radiotracer) in SPECT imaging. While ${ }^{99 \mathrm{~m}} \mathrm{Tc}-$ labelled tracers can produce higher contrast images compared to ${ }^{201} \mathrm{Tl}$ due to larger activity for the same patient radiation dose, their extraction rate is relatively low and further diminishes at high flow rates, making it difficult to detect mild ischemia $[1,2,3]$. The imaging procedure combined tread mill and diprydamole to induce stress. SPECT images were then constructed using back filtered projection and Butterworth filter [2]. The presence of ischemia was confirmed by agreement between three operators that perfusion defects exist through visual inspection of the same image. This work provided a robust approach incorporating nuclear imaging technology as a diagnostic aid to pulmonary hypertension but suffered technical limitations such as long imaging protocol and poor display. Despite the fore mentioned limitation, the work of Pereira et al provided the ground work for sufficiently determining the right ventricular functionality. 
This work provided limited insight to evaluate RV functionality, especially perfusion. Rather this work focused on visualizing the anatomy of the RV, and identification and diagnosis of PH. Consequently, this works fell short of providing tools to monitor disease progressing and efficacy of therapies.

Another closely related work by Mannting et al assessed relevance of increased trace uptake in coronary artery disease patients using RV/LV uptake ratio, using manually drawn ROIs. The paper asserts that increased RV tracer uptake is indicative of RV hypertrophy [4]. Like most studies pertaining to RV function, ${ }^{99 \mathrm{~m}} \mathrm{Tc}$-sestamibi SPECT was used because of superior contrast of ${ }^{99 \mathrm{~m}} \mathrm{Tc}$ labeled tracers compared to ${ }^{201} \mathrm{Tl}$ counterparts $[3,4]$. RV/LV uptake ratio was defined as the ratio of RV to LV activity count per pixel on the regions observed to have highest tracer uptake [3]. RV/LV uptake ratio may provide a global summary of disease, but does not provide regional information. In their work, Grapsa et al made a comparative analysis of RV volume in 3D echocardiography (3DE) and cardiac magnetic resonance imaging (CMR). Despite their work focusing on completely different modalities from PET, their work yields similar values for EDV, ESV and EF for both normal and hypertrophic hearts which provides a baseline for validating our own research. Using the Bland-Altman agreement technique, the interclass correlation (ICC) for EDV and ESV in pulmonary hypertension patients between 3DE and CMR was 0.74 and 0.75 indicating a good agreement [22]. 


\subsection{Summary}

PH mortality and morbidity is significant and the disease is poorly understood. PH plays an important role in development RV hypertrophy and subsequently dysfunction. Nuclear cardiac imaging has been applied to diseases of the LV and has resulted in sensitive tools for early disease detection, prognostication, therapy management, and tracking. These tools may be extendible to the RV. This work seeks to translate LV image analysis methods to analyze the RV in a simple, robust, adaptable, and automated method in the context of a wide spectrum of morphologies associated with pulmonary hypertension ( $\mathrm{PH})$. This tool is critical to future research including elucidation of disease related mechanisms, evaluation of therapies, and clinical management of patients.

A major portion of this research focuses on deriving the RV regions of interest which are critical to further analysis of the RV. Subsequent chapters describe the registration and sampling algorithm used to delineate $\mathrm{RV}$ region of interest in PET images and to its application in dynamic, gated, and static image analysis. Testing and validation follows in subsequent chapters along with a rigorous discussion. 


\section{Chapter: Software Development}

\subsection{System overview}

This chapter describes the development and implementation of RV functional quantification tools within the FlowQuant ${ }^{\circ}$ software package. Quantification of the RV anatomic and physiologic function is achieved through several processing steps including reorientation, registration, sampling, dynamic analysis and gated analysis. In the context of this work, these stages are defined as:

1. Reorientation - resampling of the uptake image slices from camera to standard LV reference frame. The reorientation algorithm is covered in-depth in Klein et al [6] and summarized in the background section of this document.

2. Registration - defining the region of interest (ROI) of the RV which directly depends on the LV registration as shown in the dependency diagram (Figure 3-1), because initial control points for the RV Spline model are derived from the LV sample points.

3. Sampling - defining the sampling grid using the RV ROI defined in the registration stage and sampling its image signal for display and further processing.

4. Dynamic image analysis - refers to the selection of ROIs in dynamic image sequence slices to generate time-activity curves; which are further processed to evaluate functional parameters such as metabolism and perfusion.

5. Gated image analysis - involves RV registration in all ECG-gated image volumes, which allows an evaluation of cavity volumes at different cardiac contraction phases and ejection fractions. 
The dependency diagram in Figure 3-1, illustrates the design and implementation flow of the processes. The rest of the document focuses on providing details for each step. Reorientation and LV registration processes were from the previous work by Kleinet al [6] while highlighted processes in the diagram are the focus of this research.

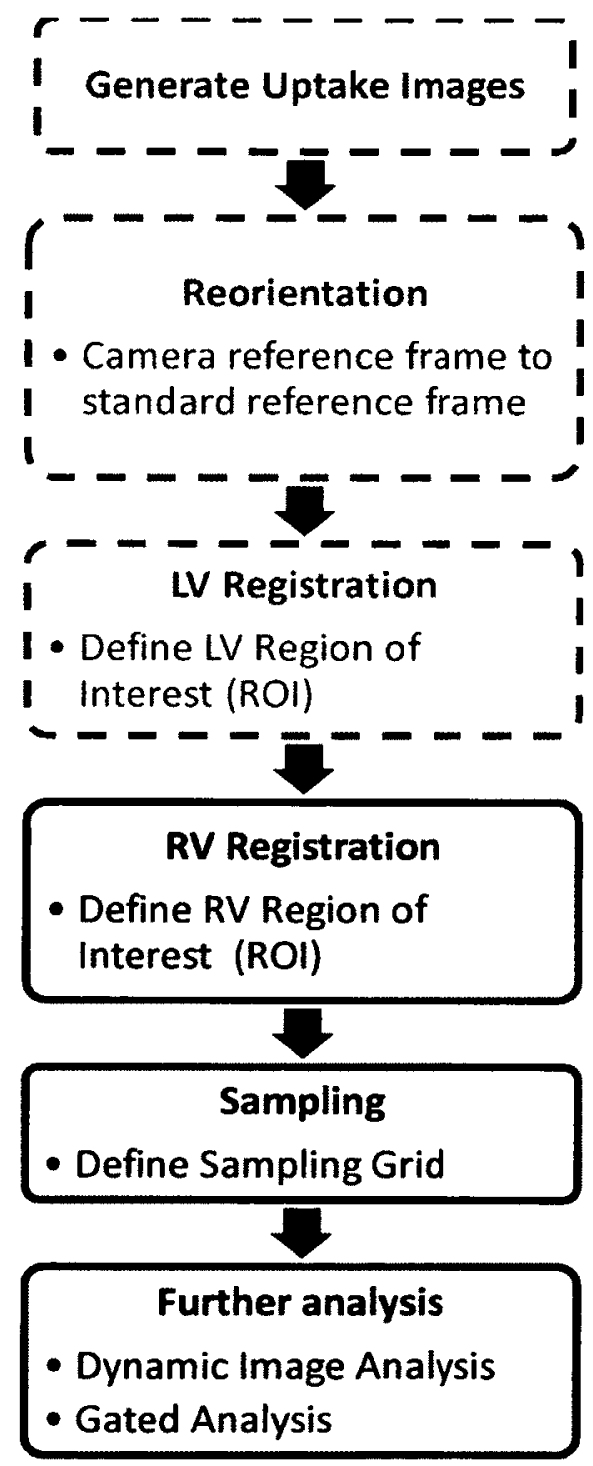

Figure 3-1: RV quantification process dependency diagram. Faded steps were previous work. 


\subsection{Reorientation}

The purpose of reorientation was to standardize cardiac PET image display since PET cardiac transaxial slices were acquired at an oblique angle; which is variable among species and individuals. Cardiac PET images were reoriented from the camera reference frame to the standard LV reference frame or LV long axis. This work depends on previous work for automatically reorienting the LV with optional operator intervention. Since the RV was reoriented along with the image volume, reorientation of the RV was inherent with LV reorientation. Section 2.4 of the background chapter provided details of LV reorientation. Therefore, the reorientation methodology was not modified.

\subsection{Registration}

\subsubsection{Overview}

The registration process defines the regions (volumes) of interest (ROI) of the left and right ventricle myocardia as well as mid LV blood cavity. In the case of medical imaging, ROI is a group of pixels to be sampled and produce a summary signal in a region of the body. While there are many possible approaches to defining ROI, the performance of each method depends on the nature of the image, the features of interest, available computational power, and degree of operator intervention, just to name a few factors. In cardiac PET imaging, organs such as the liver, stomach, and lungs can interfere due to their proximity to the heart, particularly if using surface or intensity based techniques [7]. 
Registration of the LV has been previously described by Klein et al [6] and is a convenient starting point for registration of the RV due to their combined structure. In his work, registration of the LV was done by deformable spline model [6]. Registration of the $\mathrm{RV}$ took advantage of the fact that $\mathrm{LV}$ registration has been thoroughly tested and is part of an accepted analysis tool. In this project a similar approach was used, but was significantly modified to accommodate the particular RV shape and the spectrum of morphologies in normal and hypertrophic hearts. A spline model based on a small set of control points relative to the shape and position of the LV was used. The point-based technique tries automatic registration of the RV and then implements manual adjustment, if registration fails. The spline model optimization can be constrained to favor physiologically probable RV morphologies. This section describes the RV registration process, which is the main component of the project.

The right ventricle is a closed chamber attached to the left ventricle by the septum and it is bound on the outside by the free wall. At the top is the pulmonary valve; which permits unidirectional outflow of blood to the lungs and the tricuspid valve which permits unidirectional inflow of blood from the right atrium. The RV is an irregularly shaped open vessel in cardiac PET images because valve tissues are usually not visible in the image. The RV can exhibit a range of physical shapes due to a variety of diseases, and adaptive remodeling in response to changes in workload.

The RV ROI was modeled using a spline model with a limited number of control points. The control points or spline points defined a curve; which forms the midmyocardium center line of the RV region of interest. The number of control points and their degrees of freedom account for the range of RV morphologies, Figure 2-2, that may 
be represented by the model, and were selected with computation demand and usability in mind. Estimation of the control points was done relative the LV long axis (LVLA) and then localized to the RV long axis (RVLA) for interpolation of the spline model. This implies the accuracy of RV registration depends on the accuracy of LV registration. Unlike the LV which closely maintains an elliptical shape, the RV is rather anomalous in shape when considering hypertrophic cases, and its apex tends towards the LV, hence the RVLA was defined as a curve that tapers from the mid-right atrial cavity toward the LV apex rather than a straight line as explained in the following section.

\subsubsection{Coordinate System}

Four coordinates systems were used to define the RV model and sampling points in this work. These included:

1) Image coordinates - The image represented in Cartesian coordinates $(x, y$ and $z)$ relative to the camera reference frame.

2) Reoriented Image coordinates - The reoriented image is represented in Cartesian coordinates $(x, y$ and $z)$ relative to the LV reference frame. The $x$ and $y$ pixel dimensions were dictated by the reconstructed image and the $z$ dimension was dependent on the length of the LV as determined by the reorientation stage.

3) $L V$ coordinates - The LV provided a reference point for initial estimation of the RV spline points. The LV coordinate system is a combined spherical and cylindrical coordinate system relative to the LV reference frame (reoriented image), as demonstrated in Figure 3-2. The LV apex region was represented in spherical coordinates and the cavity to atrium region was represented in cylindrical coordinates. Thus radial profiles intersect the myocardium nearly 
orthogonally in both cavity and apical regions. Sample points in the cylindrical (green) and spherical (purple) regions are shown in a long axis view (Figure 3-2A) and in the respective 3D coordinate system (Figure 3-2B and Figure 3-2C respectively). The transition between spherical and cylindrical coordinates was defined as z-slices 0 and z-slices 0,8 , and 16 were estimated as the mid LV cavity, LV base (valve plane), and mid atrium slices respectively on which the LV spline points were defined.

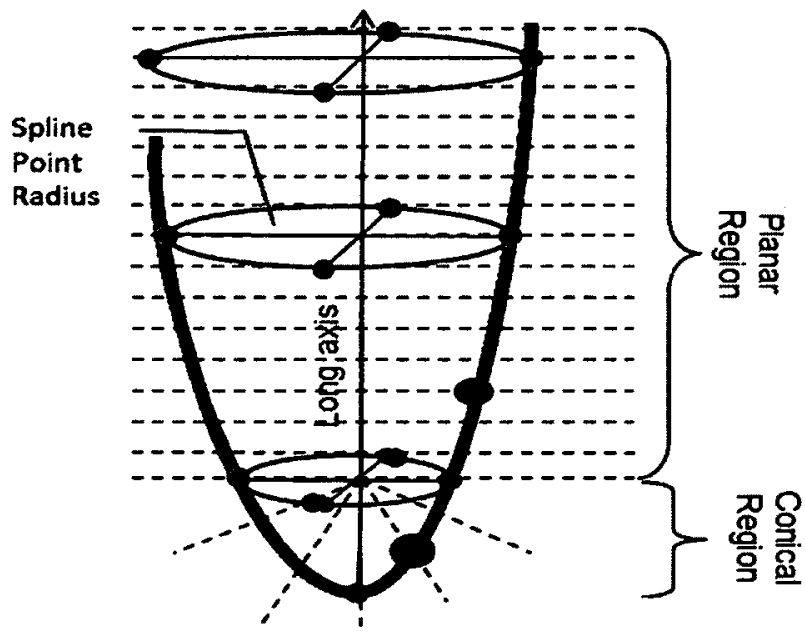

A.

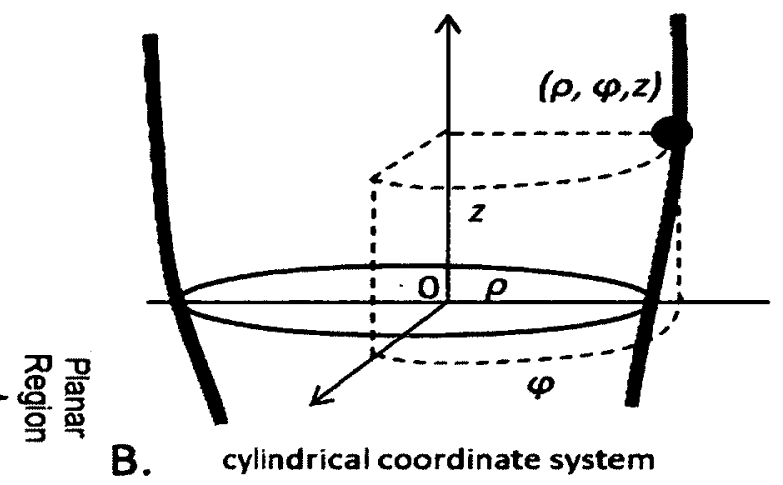

C.

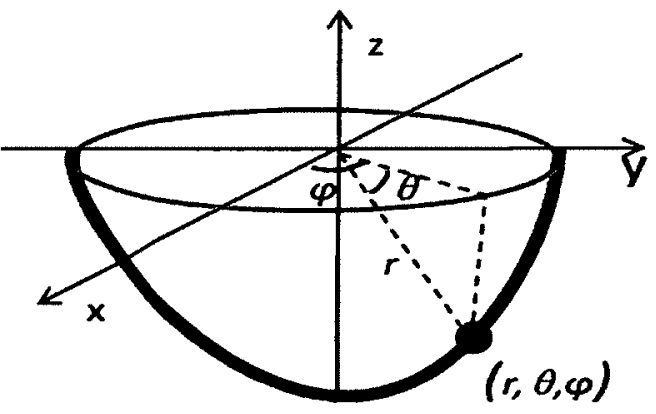

spherical coordinate system

Figure 3-2: A. Separation of the planar and conical region of the LV. B. Example of cylindrical coordinate representation of a sample point in planar region of the LV. C. Example of spherical coordinate representation of a sample point in the conical region of the $L V$. 
4) RV coordinates - A cylindrical coordinate system around the curved RVLA was used to define radials that intersected the crescent shaped RV roughly orthogonally. The curved RV long axis (RVLA) was defined from the mid atrium (z-slice 16$)$, through the mid cavity (z-slice 8 ) and to a virtual apex point, corresponding to the spline point furthest from the atrium in the $\mathrm{z}$ direction, as demonstrated in Figure 3-3 for normal and hypertrophic morphologies. This nonrigid coordinate system was selected to accommodate the wide range of RV morphologies including normal and severely hypertrophic RVs.

Spline control points and interpolated sampling points were located in $3 \mathrm{D}$ and their location represented in all coordinate systems. RV sampling points were defined in RV coordinates using a pseudo-cylindrical coordinate system, in which a polar coordinate system ( $\varphi$ and $r$ ) was defined around the RVLA in each z plane (Figure 3-3B). The RV sample points were then converted into Cartesian coordinates $(x, y$ and $z)$ and tri-linear interpolation was used to sample the image activity in the corresponding RV ROI. 


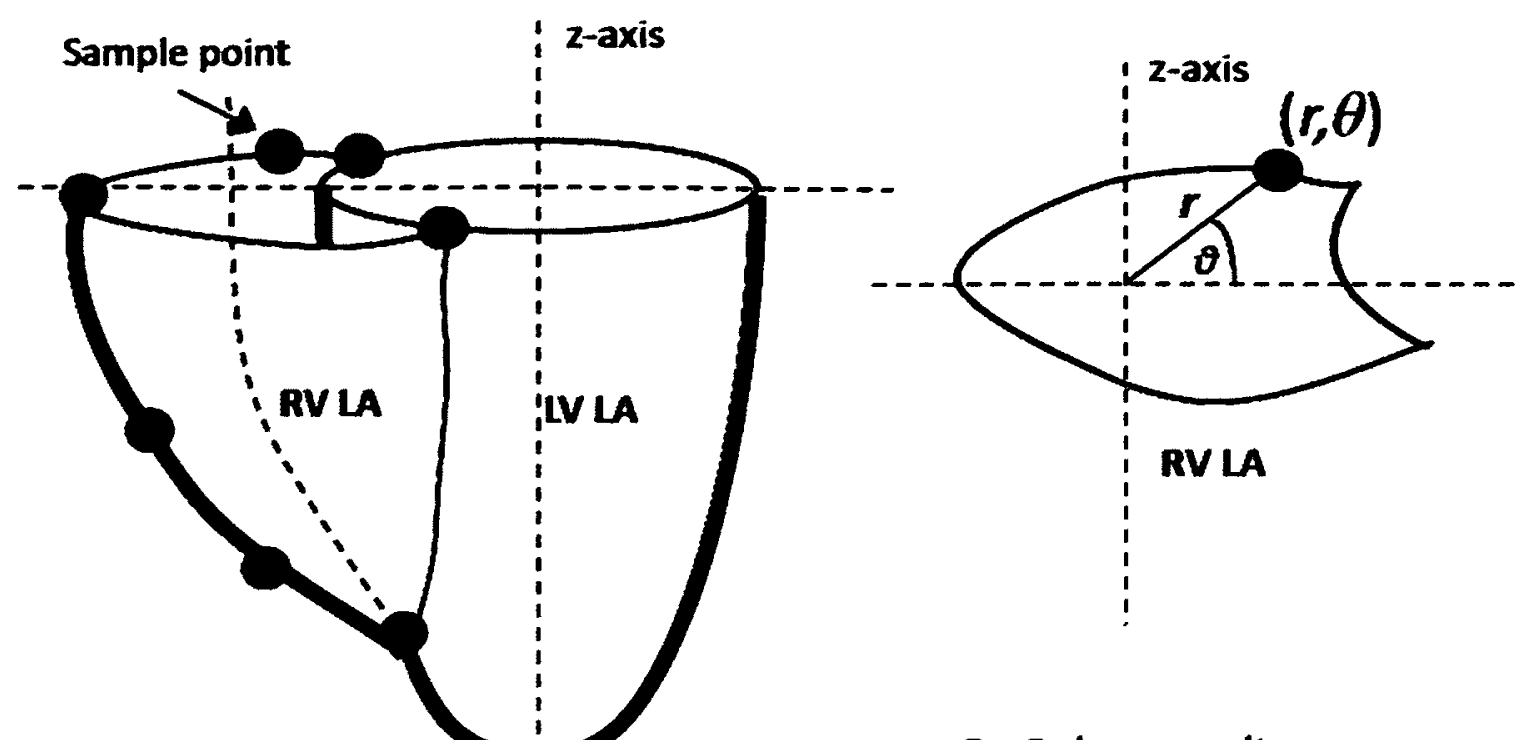

A. Normal RV

B. Polar coordinates

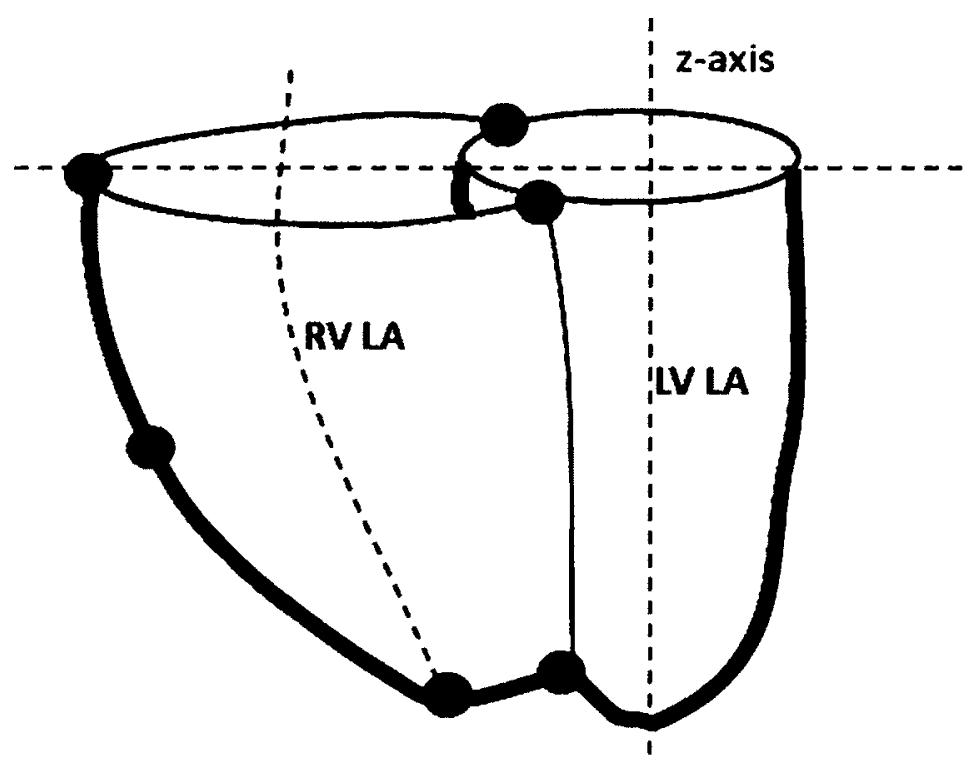

C. Hypertrophic RV

Figure 3-3 : 3D Representation of the RV and its curved long axis for both normal and hypertrophic cases, depicting derivation of pseudo-cylindrical coordinate system. B. The polar coordinate in a single z-plane. 


\subsubsection{Defining Model Shape}

In the proposed spline model 12 spline points were chosen with 13 degrees of freedom. Two short axis slices defining the mid-cavity $(z$-slice 8$)$ and the atrium ( $z$-slice 16) were each defined using 5 spline points with a single degree of freedom per spline point as shown in Figure 3-4A. Points $1 / 6$ (atrium/mid-cavity) and $2 / 8$ were the respective anterior and posterior intersect points of the RV and $L V$ and were defined as angles around the LVLA enabling tracking along the LV contour and resolving the radial distance from the LVLA based on the LV model. The notation $1 / 6$ and 2/8 implies that 1 and 2 are points in the cavity slice and 6 and 8 are points in the atrial slice. Points $3 / 8$ control placement of the free wall, and can move radially along the $x$-axis relative to the LVLA and defined the extent of the free wall and its separation from the septum. Points 4/9 and 5/10 were placed in the $x$-axis medially between the septum and free wall (i.e. on the same $x$-plane as the RVLA) and situated on anterior and posterior walls respectively. Points $4 / 9$ move radially outward from the RVLA in the positive $y$ range and 5/10 moves radially in the negative $y$ range to define the anterior and posterior extents of the RV.

All points were estimated based on LV dimensions as summarized in Table 3-1. Posterior and Anterior LV/RV intersection control points (2/8 and 1/6 in Figure 3-4A) were estimated to be $240^{\circ}$ and $120^{\circ}$ respectively for both the midsection and atrium as shown in Figure 3-4(A). As indicated in the same figure, these points were permitted to move in angular direction along the LV contour. The free wall control points (3/8) were assumed to be distance at $2 b$, where $b$ is the short axis radius of the ellipse defining LV from the reorientation stage. Points (4/9 and 5/10) were assumed to be $b$ from the RVLA and thus located medially between the septum and the free wall. 


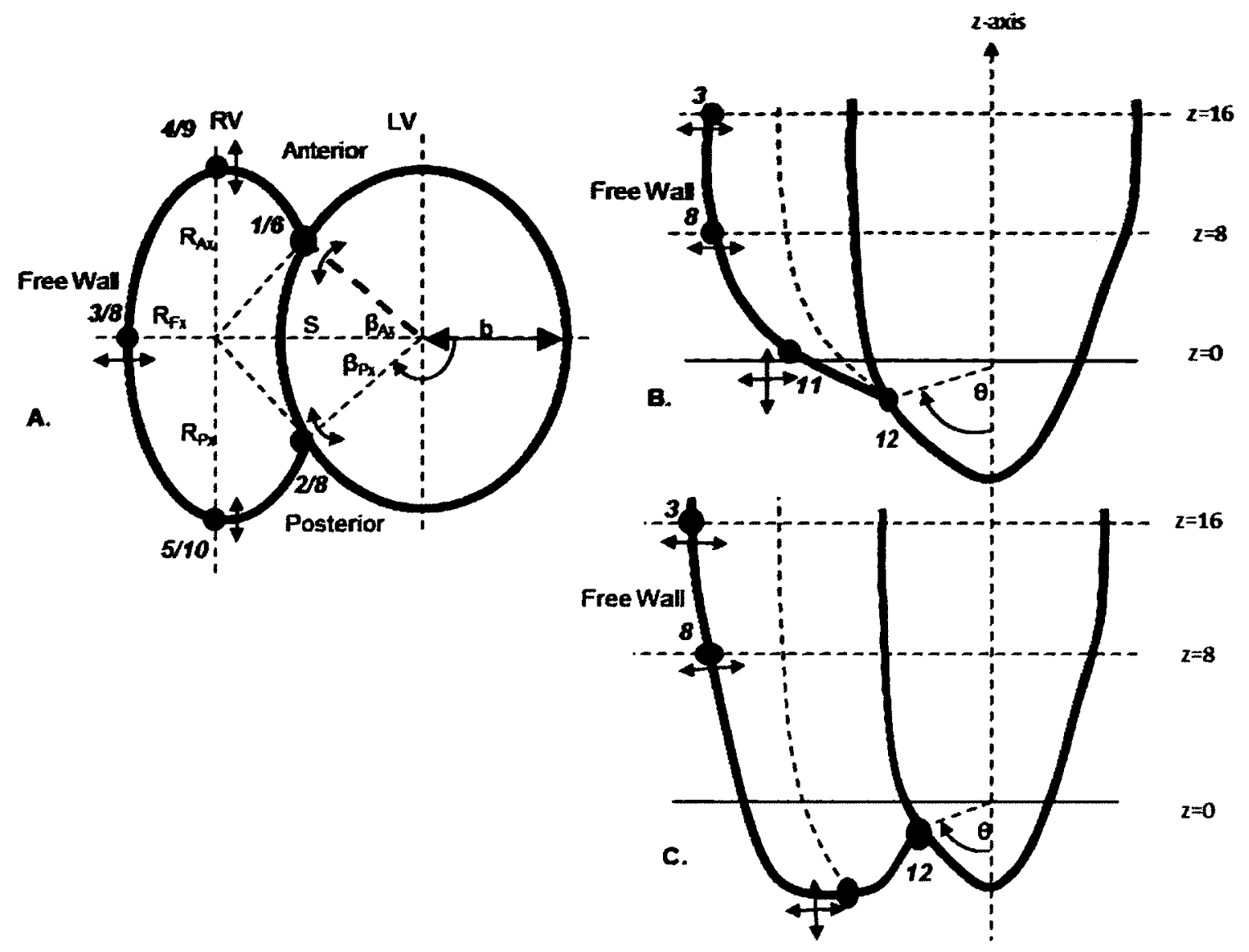

Figure 3-4 : Diagram of the RV spline model (green) relative to the previously described LV spline modell (red). Short axis slice (A) and horizontal long axis view of the model in a normal (B) and hypertrophic (C) heart. The control points are shown as black dots and described in Table 2. 
Table 3-1: Control Points and their degrees of freedom and initial location relative to the LV Long

Axis

\begin{tabular}{|c|c|c|c|}
\hline $\begin{array}{l}\text { Control } \\
\text { Point }\end{array}$ & $\begin{array}{l}\text { Degrees of } \\
\text { Freedom }\end{array}$ & Description & Initial Estimate \\
\hline 1 & 1 & $\begin{array}{c}\text { Basal Anterior RV/LV intersect control } \\
\text { point }\end{array}$ & $\beta_{\mathrm{AB}}=120^{\circ}$ around LVLA \\
\hline 2 & 1 & $\begin{array}{c}\text { Basal Posterior RV/LV intersect control } \\
\text { point } \\
\end{array}$ & $\beta_{\mathrm{PB}}=240^{\circ}$ around LVLA \\
\hline 3 & 1 & Basal Free wall spline points RV/LV & $R_{F B}=2 \times b$ from LVLA \\
\hline 4 & 1 & Basal Anterior vertical control point & $R_{A B}=b$ from RVLA \\
\hline 5 & 1 & Basal Posterior vertical control point & $R_{P B}=b$ from RVLA \\
\hline 6 & 1 & Mid Anterior RV/LV intersect control point & $\beta_{\mathrm{AM}}=120^{\circ}$ around LVLA \\
\hline 7 & 1 & Mid Posterior RV/LV intersect control point & $\beta_{\mathrm{PM}}=240^{\circ}$ around LVLA \\
\hline 8 & 1 & Mid Free wall spline points RV/LV & $R_{F M}=2 \times b$ from LVLA \\
\hline 9 & 1 & Mid Anterior vertical control point & $R_{A M}=b$ from RVLA \\
\hline 10 & 1 & Mid Posterior vertical control point & $R_{P M}=b$ from RVLA \\
\hline 11 & 2 & $\begin{array}{l}\text { RV Extent spline point -useful particularly } \\
\text { in hypertrophic case were the RV shape } \\
\text { requires more flexibility to trace. }\end{array}$ & $\begin{array}{l}\text { Midway between the RV/LV } \\
\text { intersect control point and } z=0\end{array}$ \\
\hline 12 & 1 & $\mathrm{RV} / \mathrm{LV}$ intersect control point & $\theta=90^{\circ}$ \\
\hline
\end{tabular}

Of the remaining two control points, point 12 in Figure 3-4 was referred to as the apex intersection point, and had a single degree of freedom defined as the LV/RV intersection in the apical region. It moves at angle $\theta$ with respect to the LV spherical axis along the LV contour in the HLA plane $\left(\theta=180^{\circ}\right)$, as shown in Figure 3-4B and Figure $3-4 \mathrm{C}$, and is restricted to the spherical region $\left(0^{\circ} \leq \theta \leq 90^{\circ}\right)$. The final point (point 11 in Figure 3-4), referred to as the RV extent point, has two degrees of freedom and was initially placed between the septum and the and free wall. Its ability to move horizontally and vertically in the HLA plane $\left(\theta=180^{\circ}\right)$ allows the model to accommodate different $\mathrm{RV}$ anatomies in hypertrophic cases. The extent control point is the termination point for 
the RV long axis in hypertrophic cases, where the RV extends below (lower $z$-slice number) the RV/LV apical intersection point (point 12).

\subsubsection{Defining RV Long Axis (RVLA)}

As highlighted above, the RV coordinate system was defined using a non-rigid RVLA passing through $z$-slices from the atrium to the RV extent, for which a polar coordinate system was defined in each $z$-slice. This approach enabled radii that traversed the RV myocardium nearly orthogonal, allowing optimal registration in wide range morphologies, except at the very apex of the RV.

Based on the relative $z$ position of the extent (point 11) and apical intersect point (point 12) the RVLA terminates at the point that is furthest from the atrium (lowest $z$ slice). Thus, the RVLA in normal hearts usually terminates at the apical intersection point (point 12), while in hypertrophic RVs the RVLA terminates at the extent point (point 11), as demonstrated in Figure 3-5.In the cavity and atrium planes (z-slices 8 and 16) the RVLA is positioned at $\left(\theta=180^{\circ}\right)$ and radially between the septum and free wall (see Figure 3-5). At all other $z$-slices, the coordinates of the RVLA were interpolated based on the RVLA location in the atrium plane, cavity plane, and RV extent point (point 11 or 12 accordingly).

To maintain the medial position of the RVLA in the RV cavity, the RVLA path was recomputed every time there were changes in atrial free wall, mid-cavity free wall, RV extent, and LV intersection control points (points $3,8,11$, and 12 respectively). Using RV coordinates relative to the RVLA instead of the LVLA, such that the RVLA is seemingly straight, resulted in warped RV and LV myocardial contours as demonstrated 
in Figure 3-5B and Figure 3-5D for normal and hypertrophic hearts. However this was not an issue for analysis in this thesis.

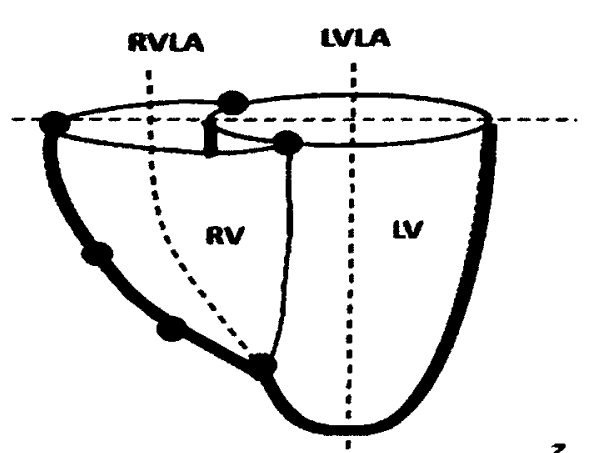

A. Normal RV

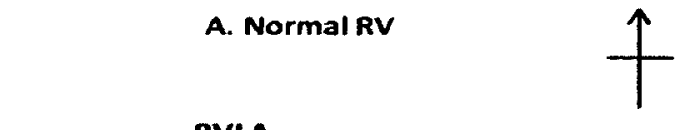

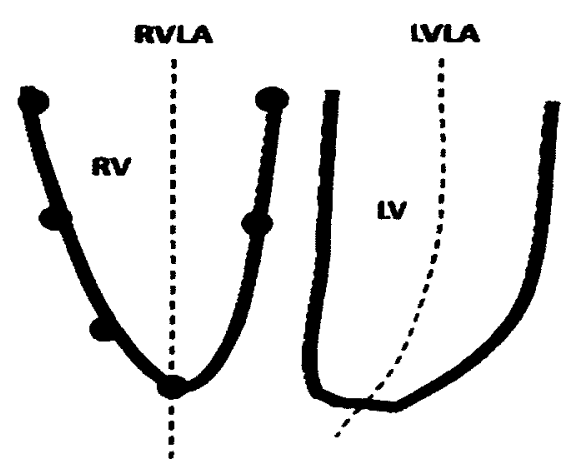

B. Morphed Normal RV and LV

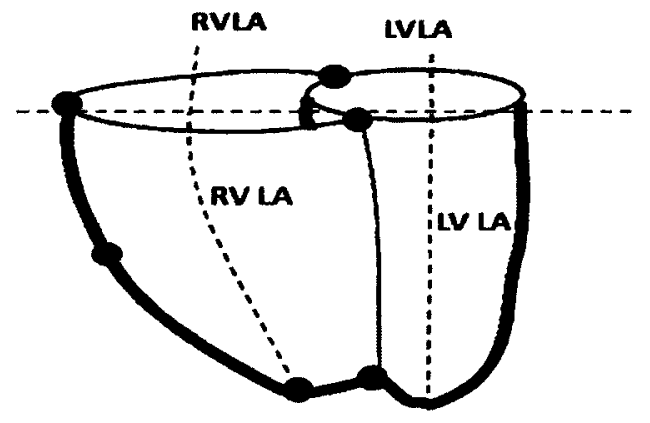

C. Hypertrophic RV

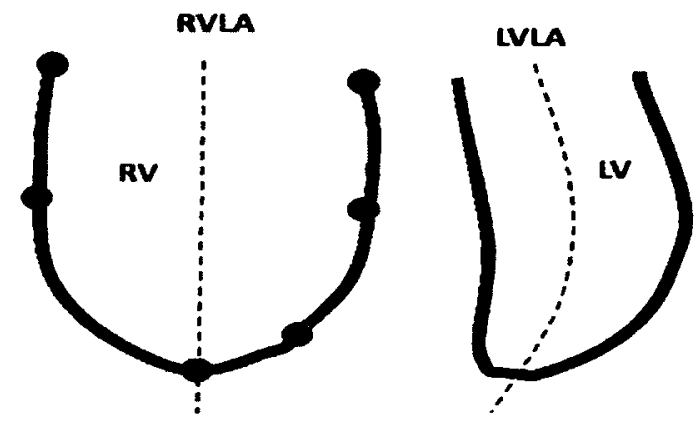

D. Morphed Hypertrophic RV and LV

Figure 3-5 (A. and C) : Illustration of the RV long axis (RVLA) in normal and hypertrophic hearts. (B and D) Morphed RV with a RVLA along the z-axis after translating control points from LVLA to RVLA for both normal and hypertrophic cases.

The anterior (points 4/9) and posterior radials (points 5/10) were located relative to the RVLA at $\theta=90^{\circ}$ and $\theta=270^{\circ}$ respectively and thus were shifted horizontally (along the $x$-axis) to remain centered between the septum and free wall whenever the RVLA was updated.

For the anterior and posterior intersection control points, which are at an angle to the LVLA, their translation to the RVLA was achieved as follows.

Variables are as defined in Figure 3-4 and Table 3-1: 
- Radius, $R_{A x}$ of the anterior RV/LV intersect from RVLA and its angle, $\alpha_{A}$ for the midsection or atrium and translation

$$
\begin{aligned}
R_{A x} & =R_{L V a}{ }^{2}+S^{2}-2 \cdot R_{L V a} \cdot S \cdot \cos \beta_{A} \\
\alpha_{A} & =\sin ^{-1}\left(\frac{R_{A x} * \sin \beta_{A}}{R_{L V a}}\right)
\end{aligned}
$$

where $b$ is the radius of the ellipse defining LV from the reorientation stage and, $x$ represent either the base or the atrium.

- Radius $R_{P x}$ of the posterior RV/LV intersect from RVLA and its angle, $\alpha_{P}$

$$
\begin{gathered}
R_{P x}=R_{L V p}{ }^{2}+S^{2}-2 \cdot R_{L V p} \cdot S \cdot \cos \beta_{p} \\
\alpha_{P}=\sin ^{-1}\left(\frac{R_{A i} * \sin \beta_{A}}{R_{L V p}}\right)
\end{gathered}
$$

\subsubsection{Sample point interpolation}

The RV sampling grid was defined to consist of 16 rings (from apex to atrium) with 18 sectors in each ring. The rings were evenly spaced in $z$ from extent point to atrium and the five SA parameters (anterior/posterior intersect angles, anterior/posterior/free wall) for each SA slice were interpolated from the control points in the atrium, cavity, and extent z-slices. In each ring, the sectors were evenly spaced angularly from anterior to posterior point of intersect with the LV. The septum was excluded as it was sampled by the LV model. For each segment a radius from the RVLA was interpolated to derive the 3D sample point coordinate (along with z-slice and angle). These RVLA coordinates was then be converted to Cartesian coordinates for sampling the RV intensity from the image. The 3D coordinates were also used to generate 3D surface images of the RV, and to compute cavity volumes and ejection fractions. Figure 
3-6A shows an example RV sampling grid. SA views of the RV ROI were created by plotting coordinates from each slice separately as shown in Figure 3-6B. HLA views of RV ROI were created by plotting coordinates of points 180 degrees from the RV LA as shown in Figure 3-6C. Trilinear interpolation of the image volume using the sampling grid was used to extract image intensities at each sample point and generate an RV polarmap as shown in Figure 3-6D.

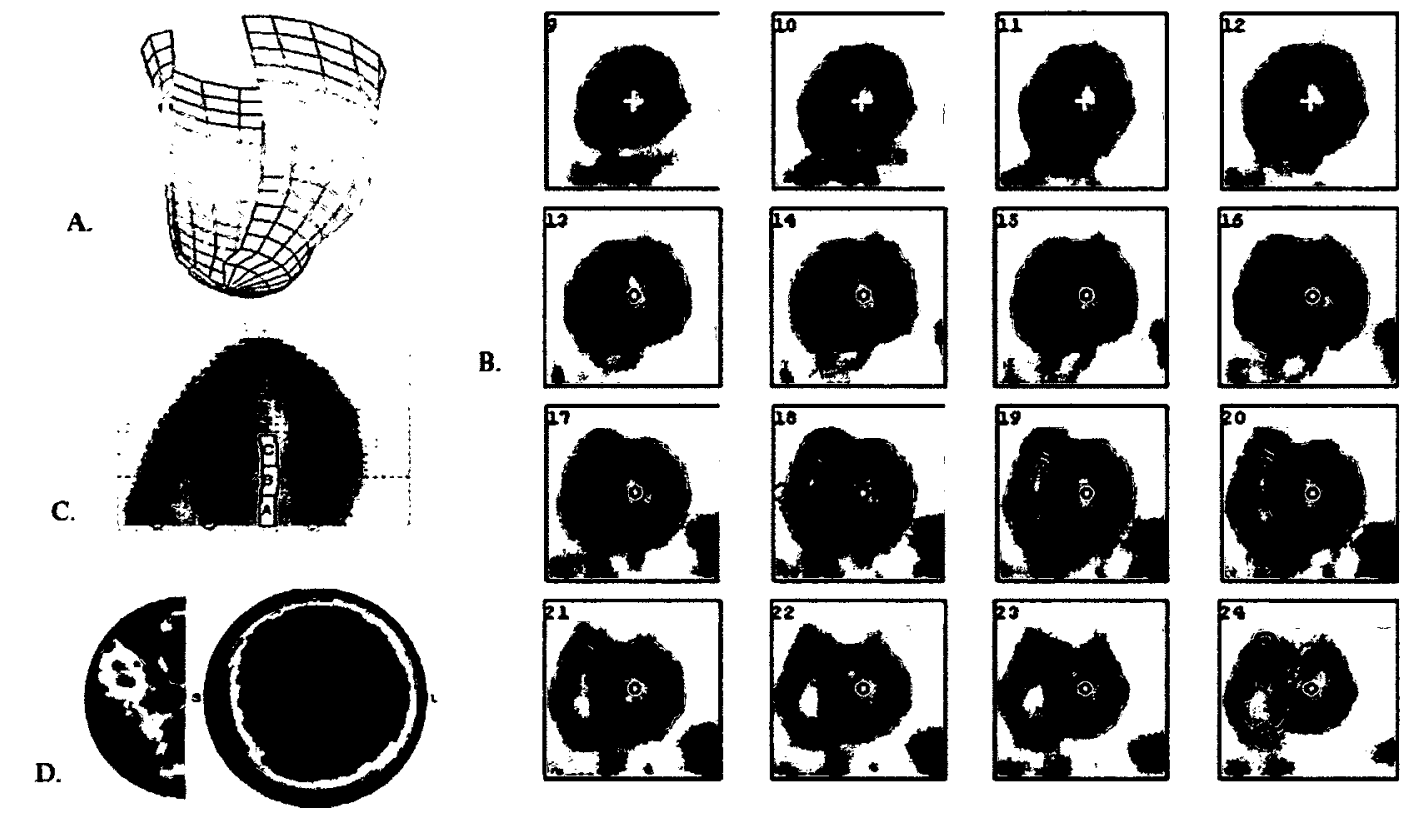

Figure 3-6 : RV ROI 3D volume generated from grid data output before sampling (A) Spline fitting model for short axis (SA) and horizontal long axis HLA (B) and (C) RV ROI is bound by blue contours. D. RV hemi polar map alongside LV polar map.

\subsubsection{Graphical User Interface Design}

Part of the work was to modify the existing graphical user interface for the LV analysis tool to allow the user to monitor RV analysis process and intervene if necessary. Major changes of the GUI included:

- Modified LVModeTool display to show the RV contours and spline points after RV segmentation (Figure 3-7) 
- Added optimize RV fit button (Figure 3-7)

- Display of the RV hemi polar alongside the LV polar map (Figure 3-8)

After automatic fitting of the spline model, the LVModelTool display shows HLA and SA slices overlaid with RV contours along with the LV contours as shown in Figure 3-7. Contours allow the user to visually inspect the accuracy of the model fit. Also in the GUI are the control points, indicated as colored circles in Figure 3-7, which can be adjusted according to their degrees of freedom if the operator perceives the fit as suboptimal. After the operator has adjusted the control points, the display will be redrawn with the operator changes applied.

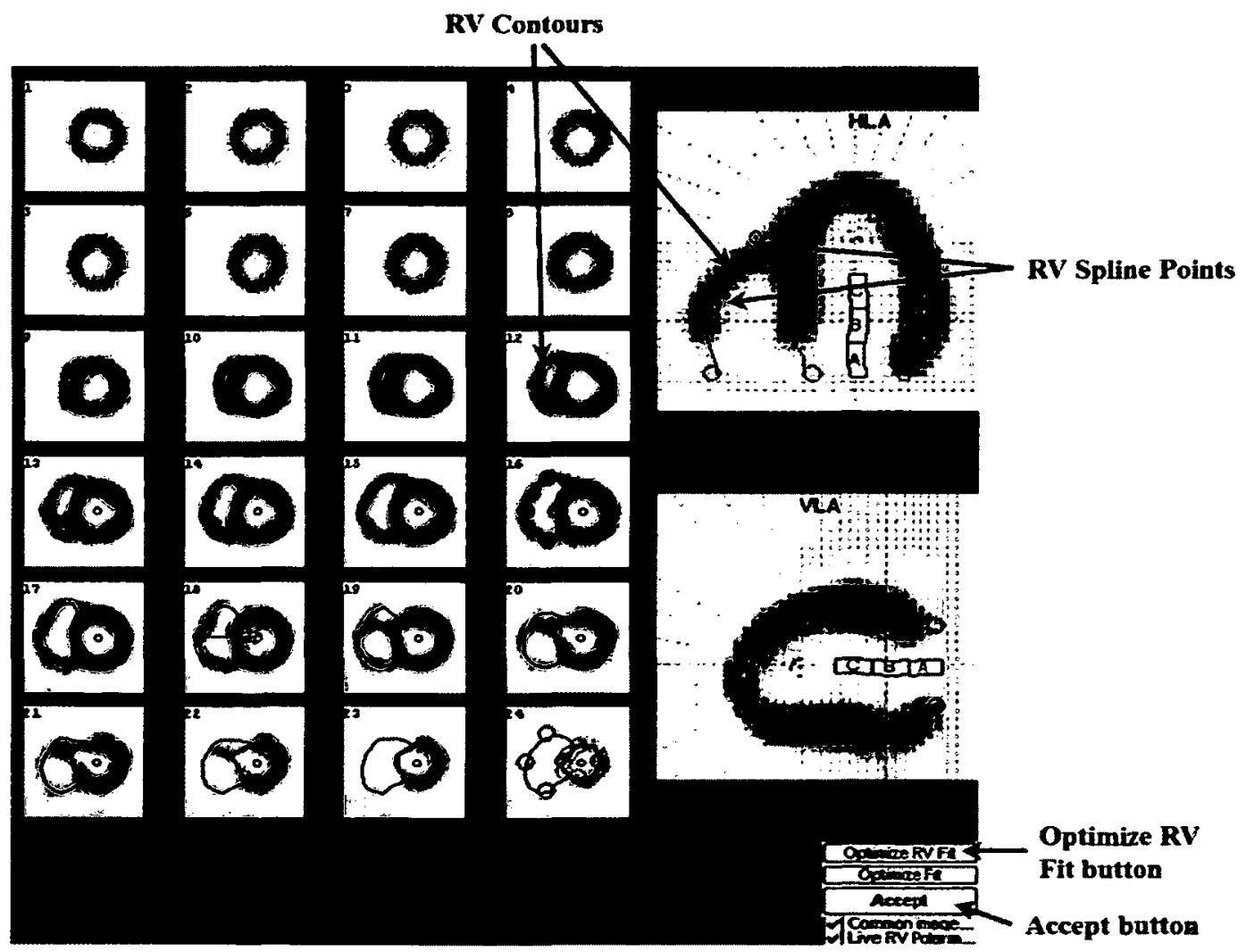

Figure 3-7 : GUI display for SA slices, HLA (top right) and VLA (bottom left) showing RV contours after registration and optimization (section 3.3.8). 
At any time the operator may invoke the automatic fitting algorithm by Optimize RV Fit button. The operator can trigger the Accept button to allow the image analysis process to permit sampling and $3 \mathrm{D}$ rendering of the ROI, if the ROI has been defined to the operator's satisfaction. The RV hemi polar and 3D surfaces were displayed alongside the LV polar maps and mesh. (Figure 3-8)
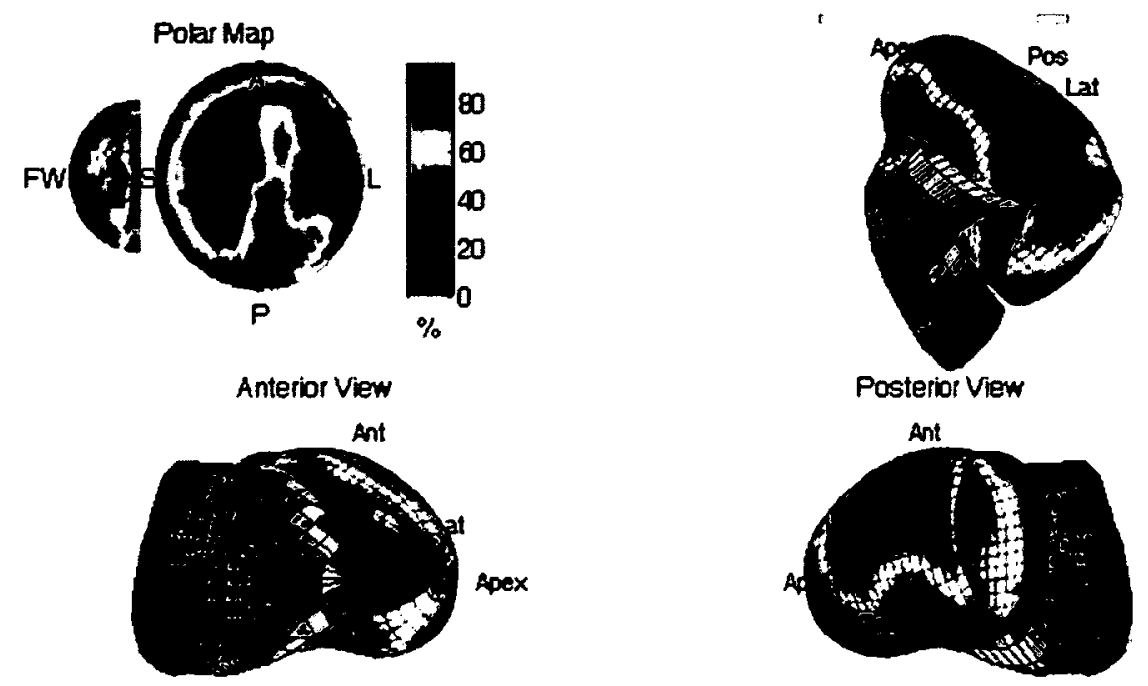

Figure 3-8 : RV hemi-polar map and LV polar map display (top left) and different views of 3D display of the RV and LV meshes

\subsubsection{Automated RV Registration}

Automation of the registration process includes estimating the initial spline points, optimization and fine-tuning. Figure 3-9 demonstrates the automated RV registration process and operator interaction workflow. 


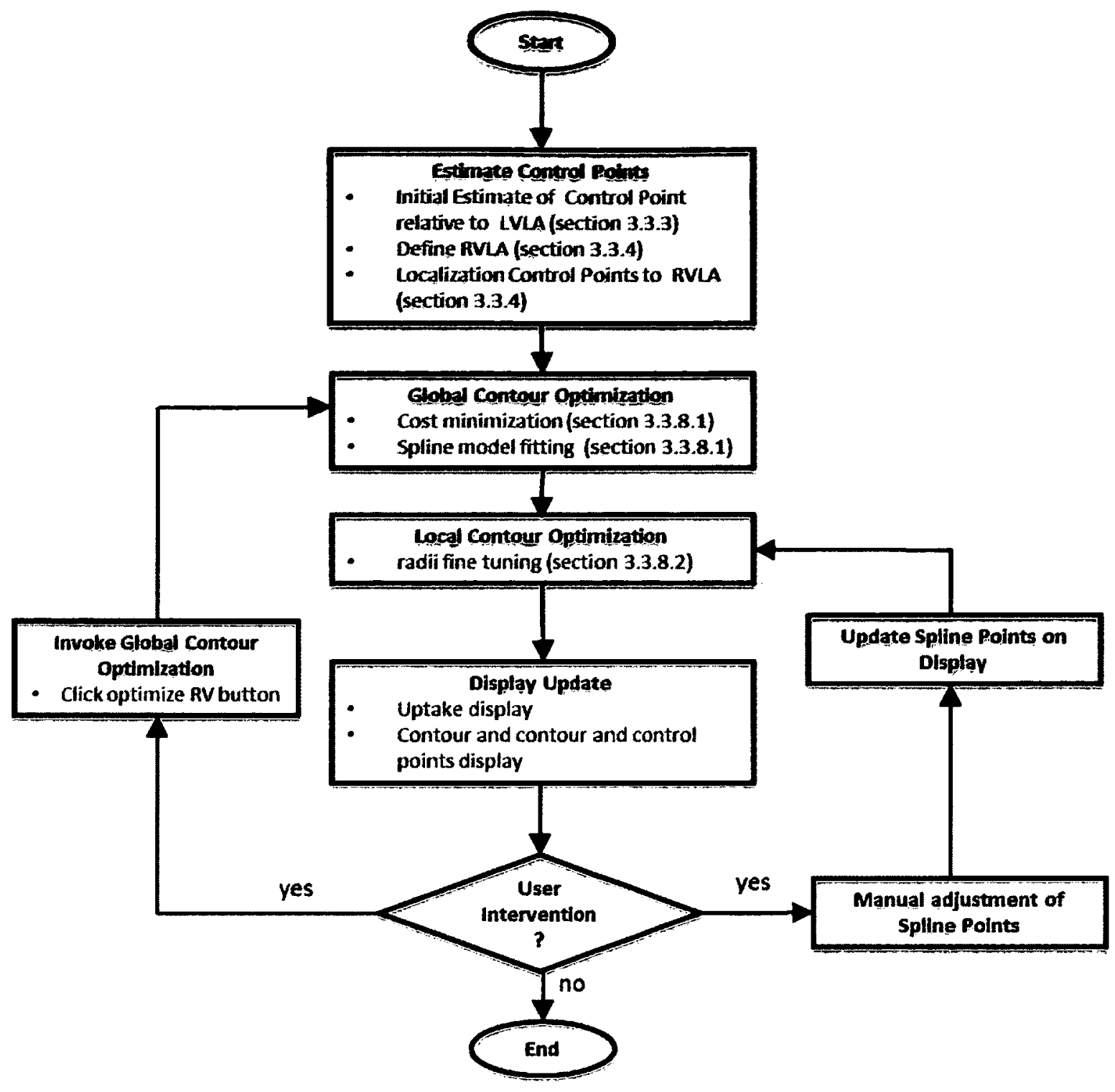

Figure 3-9 : Flow diagram for RV model registration process.

Estimation of control points involves first, defining initial starting values for control points relative to the LV long axis (points $1,2,3,6,7,8,11,12$ ), followed by defining the RV long axis, and finally redefining control points relative to the RV long axis (points $4,5,9,10$ ) (see Section 3.3.3). Spline fitting and optimization steps execute iteratively while updating control points, until a minimum value of a cost function is achieved. This is referred to as automatic registration mode. Once automation is completed, the operator 
can intervene and adjust the control points in cases where automatic optimization yields poor results, and is referred to as manual registration mode. Spline optimization can then be resumed with the current spline points serving as starting estimates, and is referred to as semi-automated registration mode. The last phase of any registration mode was local adjustment of the interpolated radii to a region of highest intensity within a search window using a center-of-mass calculation.

\subsubsection{Optimization}

Optimization employed an energy maximization method that iteratively traces the gradient of a custom cost function to locate the global local minimum [6]. The algorithm was first applied to a blurred image to mask potential local minima [6]. Blurred images were created by applying a predefined 3D Gaussian smoothing to the original uptake image to achieve 24 and $4 \mathrm{~mm}$ FWHM image resolution for humans and rats respectively [6]. RV shape estimates from the blurred image, are used as initial estimates for fitting the same spline model to the original uptake image. Optimization progressively deforms the shape of the estimated RV surface maximizing the image intensity while adhering to the assumed shape of the RV as defined by the spline model. In semi-automatic mode, the operator can set the initial positions of control points (circular makers in Figure 3.7) and restart the optimization process using the original uptake image. The following text provides details explanations of the custom cost function as well as subsequent optimization of the location of individual sample points.

\subsubsection{Global Contour Optimization}

An iterative optimization algorithm (fminsearch, Matlab, Natick, MA) was used to adjust the control points to automatically register the RV myocardium by minimizing a 
custom cost function taking control points $1,2,3,6,7,8,11,12$ as input parameters. The cost function, $\mathrm{C}$, was derived from two cost components; a cost incurred when attempting to maximize the sampled image intensity, $C_{\text {intensity, }}$ and a cost incurred when the constraints on assumed shape or size of the RV were violated, $C_{C}\left((3-5) . C_{C}\right.$ was a cumulative cost derived from a number of cost functions, each function penalizing a specific constraint violation as indicated by the equation 3-6 .

$$
C=C_{\text {intensity }}+C_{C}
$$

where

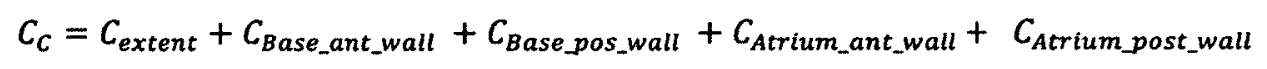

All components of the cost function and constraints are explained below:

1) Maximization of sampled image intensity

During model optimization the algorithm seeks the region with highest aggregate intensity overlapping with the RV ROI as defined by the model [6]. The model applies a cost minimization function that imposes low cost on ROIs sampling high image intensities, and imposes a high penalty for low intensity ROIs [6]. The cost minimization function is defined as follows for all pixels $p$ of the ROI,

$$
C_{\text {intensity }}=\sum_{p \in R O I} \frac{I_{\max }}{I_{\max }-I_{p}}
$$

$I_{\max }$ is the maximum image intensity and $I_{p}$ is the image intensity of pixel $p$.

2) Constraints on RV shape and size

The constraints based cost penalized when either the assumed size or shape of the $\mathrm{RV}$ were violated. The bounds of the size and shape of the RV were defined by 
visual inspection of a representative set of images including normal and hypertrophic RVs of rats and humans. These constraints consisted of:

- Extent control point - the extent control point controls the curvature and extent of the RV along the $z$-axis. During optimization the point was fixed midway between the septum and free wall and was allowed to move along the $z$-axis with the cost function shown in equation 3-8

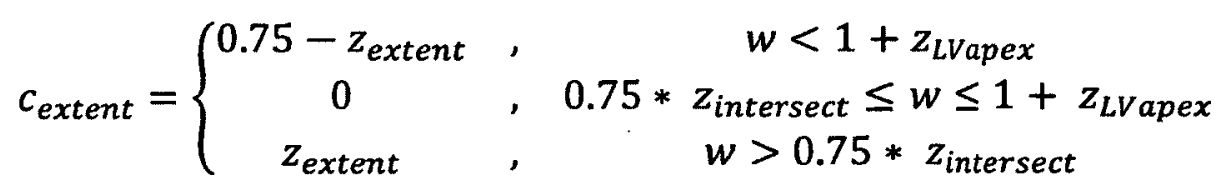

where $z_{\text {LVapex }}$ is the $z$-coordinate of the LV apex and $z_{\text {intersect }}$ is the $z$-coordinate of the LV/RV intersect point as depicted in Figure 3-10.

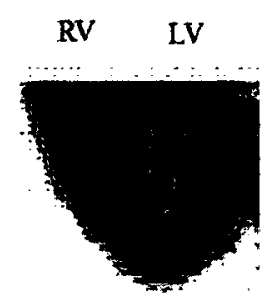

Nomal RV

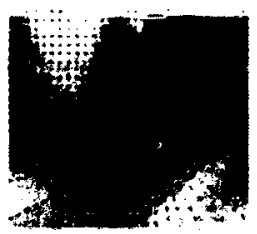

A. Hypertrophic RV

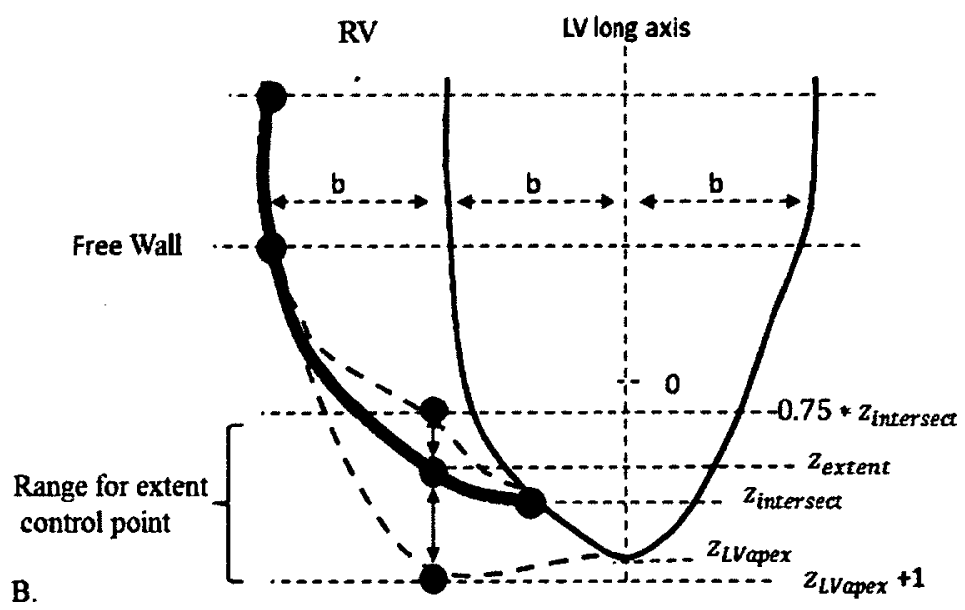

B.

Figure 3-10 : Deducing the bounds for extent control point. A. Extent control point in normal and hypertrophic cases. B. Range of the extent control point.

- The free wall was constrained at both the atrium and mid-section. A position parameter, $w_{l}$, was calculated according the equation 3-9, where $R_{1}$ is the distance of the free wall (either at the base or atrium) from the LVLA and $b$ is the short axis radius of the LV ellipse from 
reorientation stage. The costs incurred when constraints were violated were evaluated using equations $3-10$ and 3-11 for the base and atrium respectively.

$$
\begin{gathered}
w_{1}=\frac{R_{1}-b}{R_{1}} \\
c_{\text {Base }}=\left\{\begin{array}{cc}
0.45-w_{1}, & w_{1}<0.45 \\
0, & 0.45 \leq w_{1} \leq 0.65 \\
w_{1}, & w_{1}>0.65
\end{array}\right. \\
c_{\text {Atrium }}=\left\{\begin{array}{ccc}
0.2-w_{1}, & w_{1}<0.45 \\
0, & 0.2 \leq w_{1} \leq 0.65 \\
w_{1}, & w_{1}>0.65
\end{array}\right.
\end{gathered}
$$

- Anterior and Posterior wall positions were also constrained at both the atrium and base. The position parameter, $w_{l}$, for this constraint was determined using equation 3-12 where $R_{2}$ is the vertical distance of either the anterior or posterior wall from the RVLA. The equation was applied both at the atrium and midsection, and specific limits for $w$ and associated penalties in each region were calculated as in equations 314,3-15 and 3-16 and as follows :

$$
\begin{gathered}
w_{2}=\frac{R_{2}-b}{R_{2}} \\
c_{\text {Base_ant_wall }}=\left\{\begin{array}{cc}
0.01-w_{2}, & w_{2}<0.01 \\
0, & 0.01 \leq w_{2} \leq 0.6 \\
w_{2} & w_{2}>0.6
\end{array}\right. \\
c_{\text {Base_pos_wall }}=\left\{\begin{array}{cc}
0.01-w_{2}, & w_{2}<0.01 \\
0, & 0.01 \leq w_{2} \leq 0.5 \\
w_{2}, & w_{2}>0.5
\end{array}\right.
\end{gathered}
$$




$$
\begin{aligned}
& c_{\text {Atrium_ant_wall }}=\left\{\begin{array}{ccc}
0.08-w_{2} & , & w_{2}<0.08 \\
0 & , & 0.08 \leq w_{2} \leq 0.5 \\
w_{2} & , & w_{2}>0.5
\end{array}\right. \\
& c_{\text {Atrium_post_wall }}=\left\{w_{2} \quad, \quad w_{2}>0.5\right.
\end{aligned}
$$

\subsubsection{Local Contour Optimization}

Local contour optimization followed the model optimization stage as an optimization of the radial position of each sample point individually, thus accommodating RV shapes that don't strictly follow the spline model. The radial position of each sample point was optimized to pass through the center of mass of the myocardium within a radial search window as shown in Figure 3-11. The search window was centered at the radius determined by the spline model and was 11 pixels wide ( 5 pixels on either side) to generate an activity profile through the myocardium.

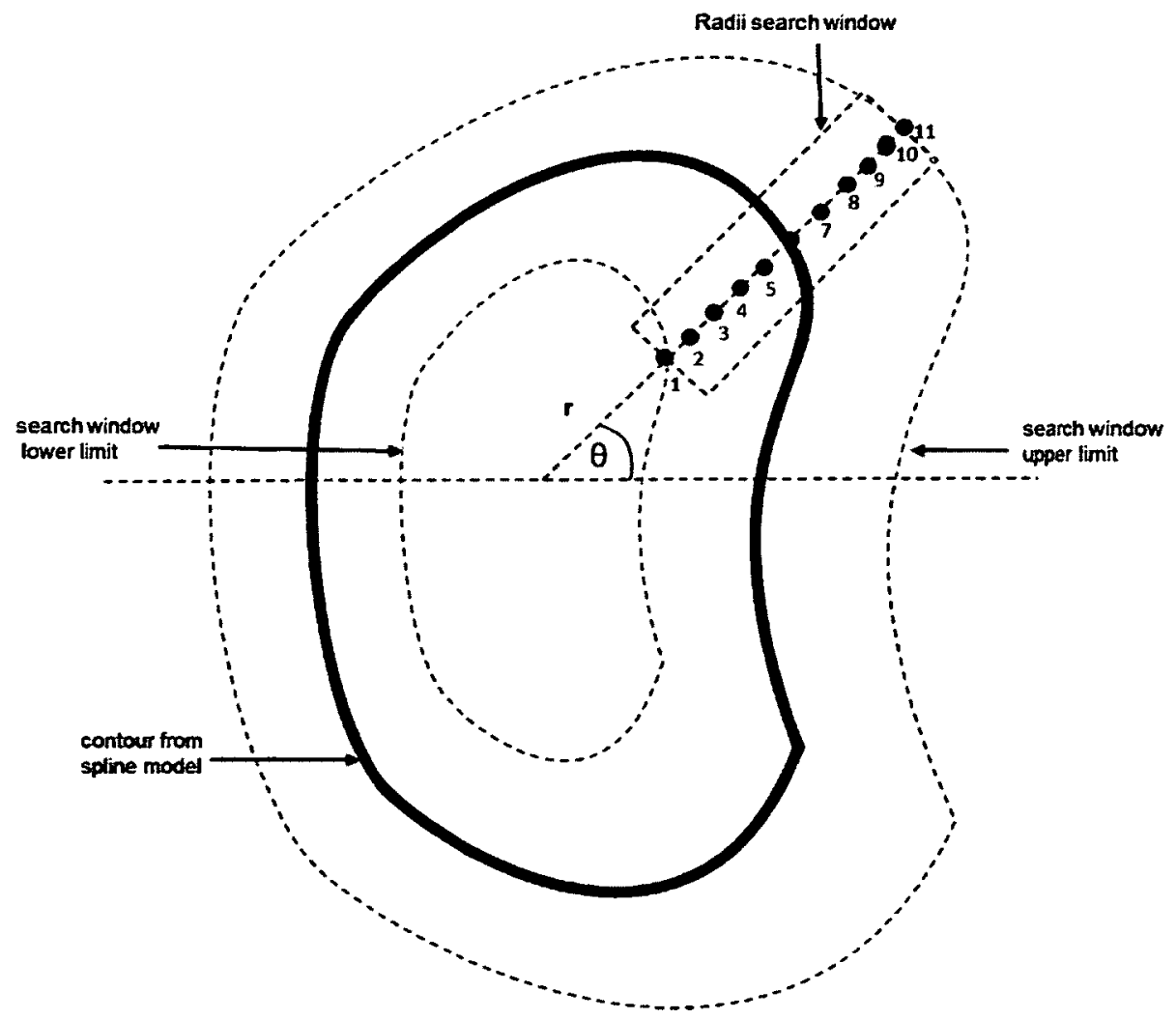

Figure 3-11 : Sample slice RV slice showing fine tuning search window, boundaries of the search 
Each profile was independently processed to locate the mid-myocardium radius $r_{c s}$ using a weighted center of mass calculation as shown in equation 3-17 where $I_{p}$ is the image intensity and $r_{p}$ is the radius of all pixels $p$ of the profile.

$$
r_{c s}=\frac{1}{\sum_{p \in M} I_{p}} \sum_{p \in M} r_{p} I_{p}
$$

\subsection{Sampling}

The RV sample point defined in radii, angles, and $z$-slices according to the RVLA coordinate system were converted to Cartesian $(x, y$ and $z)$ coordinates in the reoriented image. Complete RV sampling consisted of 16 rings evenly spaced in $z$-slices between the apex and atrium, and in each ring the 18 sectors were evenly spaced angularly from the anterior to the posterior intersect with the LV. The complete RV sampling grid therefore consisted of 288 sectors. The Cartesian coordinates were then be used for trilinear interpolation of the activity of each RV segment from the image data. The sampled data was used for display of 2D polar maps of the tracer activity/concentration in the PET image, as well as for further analysis (e.g. kinetic modeling).

\subsubsection{Polar map display}

RV polar map sampling was modified from the work of Chiba et al [52] which used standard cylindrical sampling. A RV hemi-polar map, as shown in Figure 3-12, was obtained by plotting the sampled data in polar coordinates, such that the RV atrium was mapped to the outer rings of the hemi-polar and the RV apex was mapped to the center. The free wall was mapped to the left of the hemi-polar with the septal intersection forming a straight line to the right, and the anterior and posterior walls were positioned at the bottom and top respectively. 

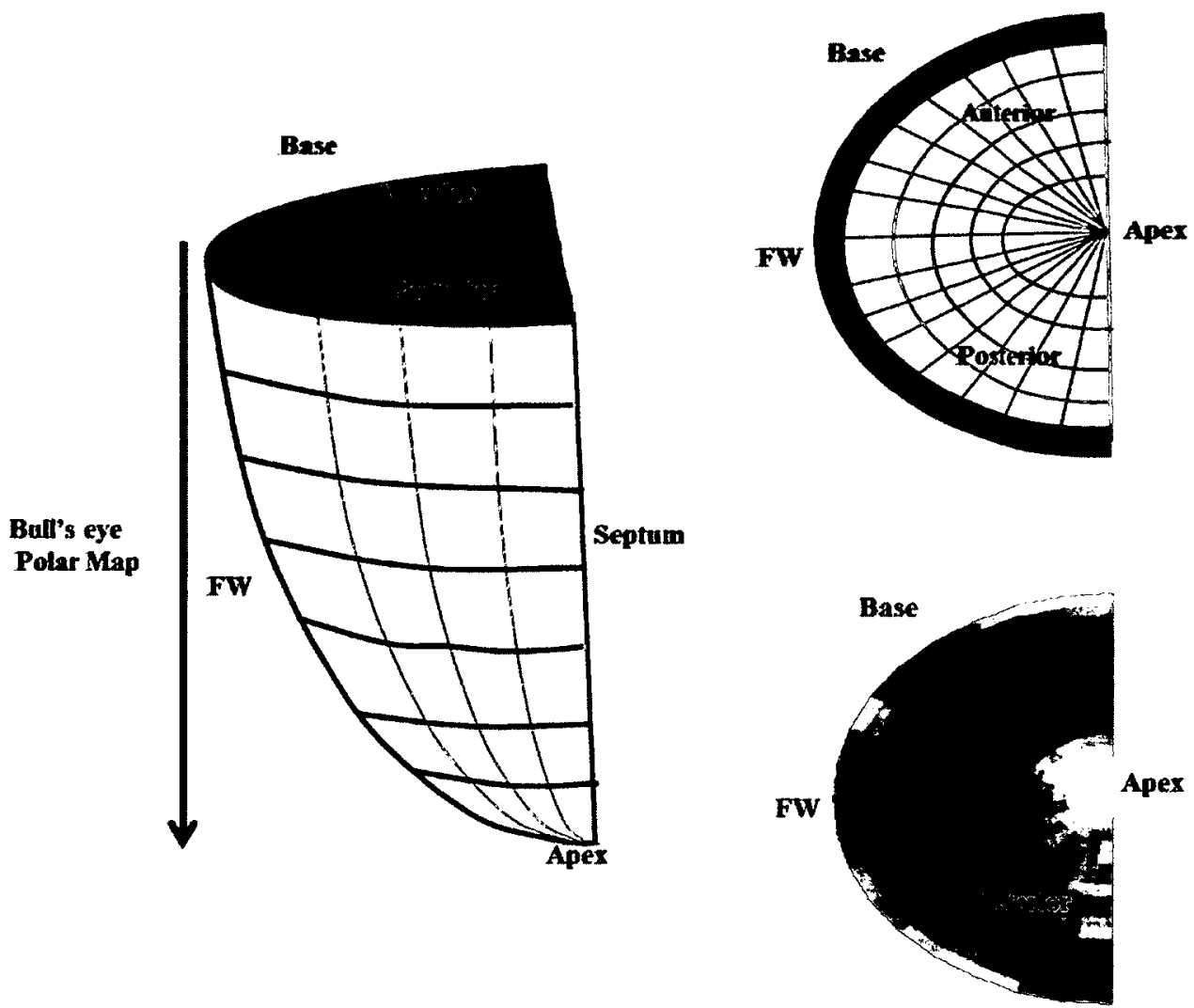

Figure 3-12 : Cylindrical polar map sampling to create a hemi polar. Direction of the view of the polar map (base to apex) (Left). A sample slice divided into equal sectors (top right). A hemi polar created from cylind rical sampling (both right). FW - free wall

\subsection{RV segmentation}

As with the LV, the RV region of the hemi polar map were segmented into different regions, for more localized statistical analysis. For further analysis we elected to segment the polar map using a 3 segment model, excluding the right atrium RV/LV intersections including $\mathrm{RV} / \mathrm{LV}$ posterior and anterior intersections and $\mathrm{RV} / \mathrm{LV}$ apical intersection as shown in Figure 3-13. The intersection regions were excluded due to susceptibility to interference from LV signal due to spillover effects. The image intensity in the atrial region tends to sharply decrease making the accuracy of the spline model fit 
questionable. These 3 vascular regions are proximal, medial and distal (Figure 3-13). This division of the hemi polar allowed a more precise validation of the performance of the spline model especially in operator variability testing.

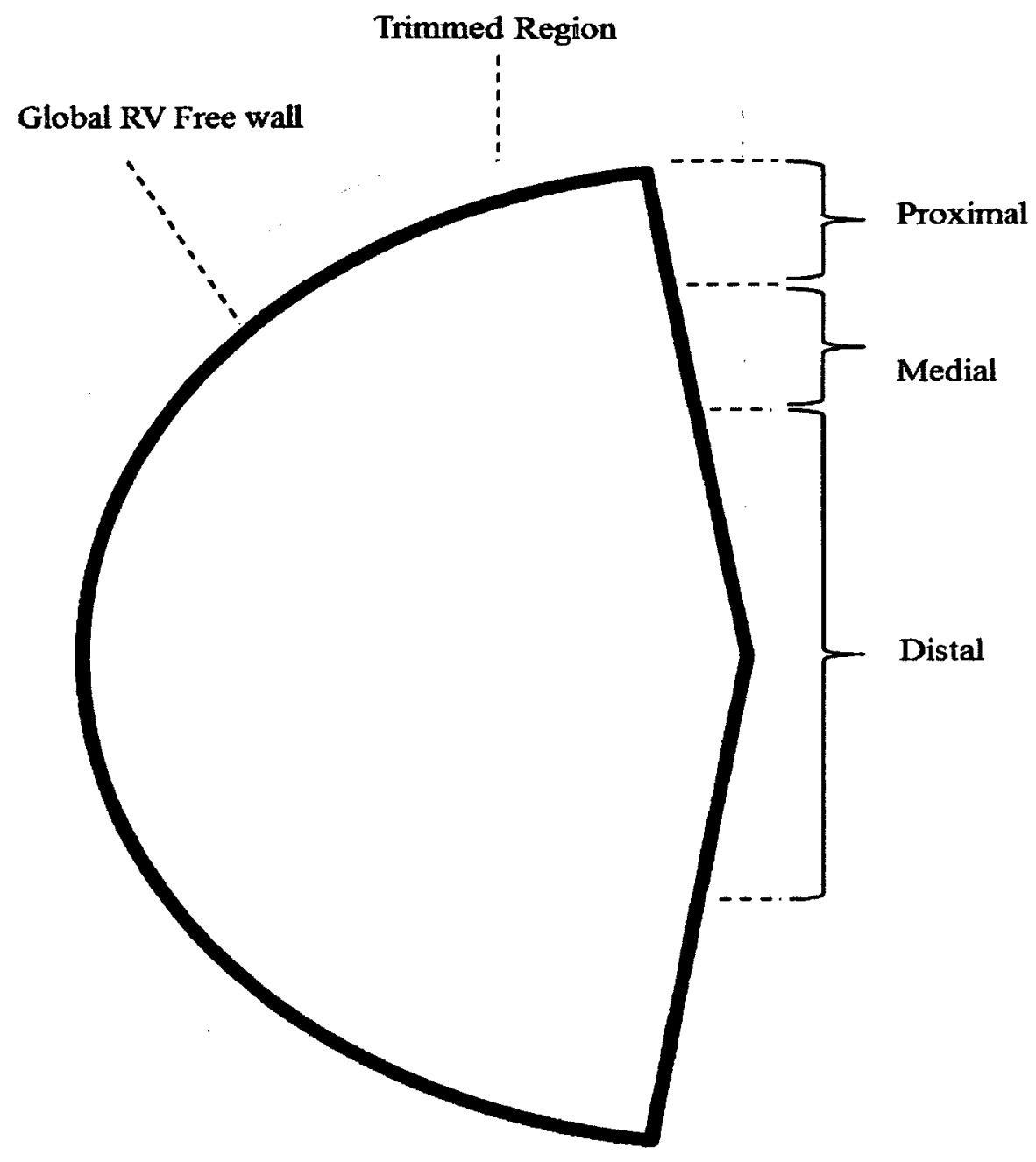

Figure 3-13 : Three vascular segments of the RV hemi polar. The trimmed region represents regions are unlikely to produce reliable physiologic measurements, including the RV/LV intersection regions which are susceptible to LV spillover, and the atrial region where the thin atrial wall and low image resolution result is low image intensity. 


\subsection{Gated Image Analysis}

The purpose of gated analysis was to evaluate cavity volumes and ejection fraction which can be used to determine the efficiency of the RV as a pump. As described earlier in the background section, the gated images correspond to several (8-32) phases of the beating heart cycle.

Gated analysis began with averaging of all the gated images to generate an average motion blurred image, but with less statistical noise than any individual gate. The spline model was applied to the averaged image to generate an initial estimate of the RV model. Using the estimation from the average image, every image from each gate was automatically processed to register a gate specific RV model.

For each gate, the cavity volume was estimated by binary masking of pixels inside the RV contour. The normalized volume was estimated as a sum of the masked pixels. The final volume in milliliters was obtained by multiplying the normalized volume by the pixel dimensions.

In order to account for the finite myocardial wall thickness, prior to volume calculation, the radii for the RV ROI volume are decremented by an estimated wall thickness, thus factoring that the RV ROI is a mid-myocardial curve rather than the endocardiac border. Likewise in the septal region, the LV radii are increased by the same assumed wall thickness. The end-diastolic wall thickness was assumed to be $5 \mathrm{~mm}$ in humans or $5 \mathrm{~mm}$ in rats. In all other gates, the wall thickness was adjusted to preserve the myocardial muscle volume, as the tissue is assumed to be non-compressible. Thus, the myocardial wall thickens increases as the heart contracts. 
The gate with the largest cavity volume was assumed end-diastole and the gate with smallest volume assumed end-systole. The corresponding end diastole volume (EDV) and end systole volume (ESV) were used to estimate the RV ejection fraction as the percentage ratio of the difference between the EDV and ESV and EDV as shown in the equation below.

$$
E F=\frac{E D V-E S V}{E D V} * 100 \%
$$

The cavity volume for the RV of the beating heart was plotted alongside the LV cavity volume as demonstrated in Figure 3-14. Also shown in the complete gated analysis report are 3D meshes of the beating LV and RV generated along with polar maps.

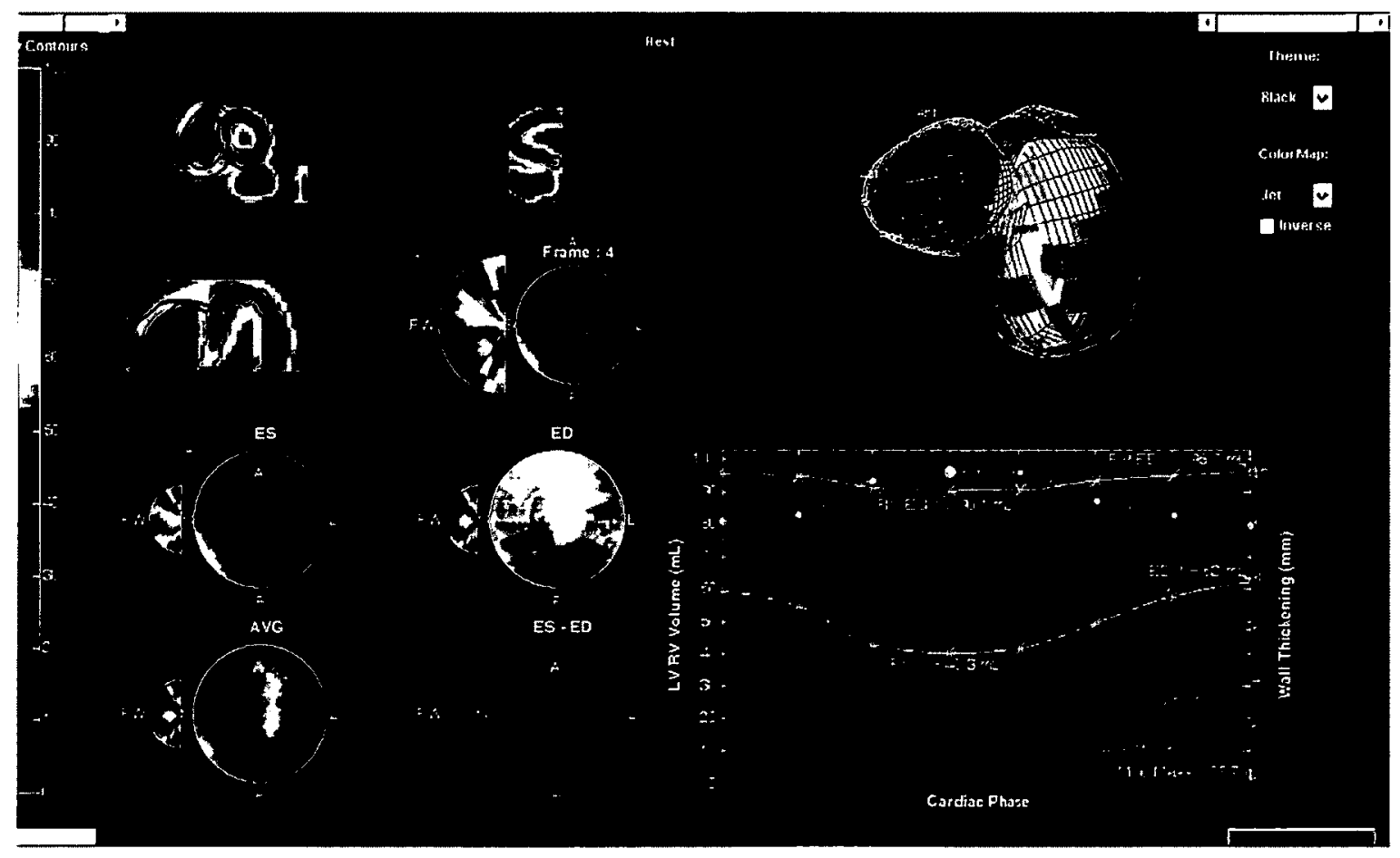

Figure 3-14 : Gated analysis report showing plot of RV Cavity volumes and EF for a hypertrophic $R V$ along with RV and LV 3D meshes and polar maps of beating heart. 


\subsection{Summary}

This chapter described a novel method for generating RV regions of interest with minimal operator intervention and sufficient flexibility to accommodate a wide range of RV morphologies. A complete software solution was integrated into the FlowQuant ${ }^{\odot}$ software package to facilitate future research using RV imaging by non-expert users. This solution included analysis of static images to generate $2 \mathrm{D}$ polarmaps that can be used to visualize relative regional patterns in the $\mathrm{RV}$ and tracer concentration relationships between the RV and LV. Gated images were processed to analyze wall motion, RV cavity volumes and ejection fractions. Dynamic images were processed using a complete set of kinetic and graphical analysis tools already existing for LV analysis to estimate bio-chemical rate parameters of the tissue. The following chapter deals with validating, adjusting, and characterizing these methods. 


\section{Chapter: Validation and Characterization}

The previous chapters provided design and implementation details of the spline model. This chapter provides a formal validation and characterization of the implementation. The appropriateness of the spline model was evaluated for its ability to correctly register the RV ROI in representative images. The first evaluation assessed the model's ability to accurately define RV ROIs using manual adjustment of the control points. The second part of evaluation involved assessing the correctness of RV registration when control points are automatically adjusted and optimized. Operator variability was then characterized to assess reproducibility of measurements with the software implementation. The degree of automation and operator variability when applying the model was analyzed to characterize its behavior. The procedure below outlines the steps taken to validate and characterize the spline model. Finally, the accuracy of the software for measuring RV cavity volumes and ejection fractions was evaluated.

\subsection{Appropriateness of the Model (Manual fitting of control points)}

The model was manually fitted to determine its appropriateness for defining the RV ROI. The quality of fit for each image was evaluated by the operator through visual inspection. Each control point was manually adjusted until the operator was content with the model fit. A pass was granted when the model was judged by the operator to sufficiently trace the RV mid-myocardium and appropriately intersect the LV as visualized by the GUI. 


\subsubsection{Study population}

A dataset consisting of 5 non-pulmonary hypertension (non-PH) human, 5 confirmed pulmonary hypertension ( $\mathrm{PH})$ human, 5 baseline rat and 5 follow-up rat cardiac FDG PET images were used to evaluate the appropriateness of the proposed RV spline model. The study population characteristics are summarized in Table 4.1.

. For all human subjects a written informed consent was obtained from all participants based on UOHI ethics guidelines.

Non-pulmonary hypertension subjects were randomly selected clinical patients with unconfirmed cases of $\mathrm{PH}$ and possible suffering from other cardiac diseases. These patients were all part of a registry study and provided informed consent to participate in retrospective studies. 5 confirmed pulmonary hypertension human subjects were part of an ongoing study at the Ottawa Heart Institute on Right Ventricular Substrate Metabolism as a Predictor of Right Heart Failure in Patients with Pulmonary Arterial Hypertension. The recruitment criteria for subjects included patients with right atrial pressure of at least $14 \mathrm{mmHg}$ measured using a right heart catheterization pressure probe [56].

Baseline rats included Adult male Sprague-Dawley rats weighing between 150 and 200 grams. Follow-up rats images were acquired about 4 weeks after baseline rats were injected with monocrolatine, a pulmonary hypertension inducing agent [50]. All animals were handled in accordance with the Guide for Care and Use of Laboratory Animals and the University of Ottawa Animal Care and Use Committee. 
Table 4-1: Population characteristics for subjects used in validation.

\begin{tabular}{|c|c|}
\hline $\mathbf{P H}(\mathbf{N}=5)$ & Mean \pm SD \\
\hline Age (years) & $60 \pm 13$ \\
\hline Gender (female) (N) & 4 \\
\hline weight (kg) & $71.2 \pm 14.72$ \\
\hline height (m) & $1.63 \pm 0.10$ \\
\hline \multicolumn{2}{|l|}{ non-PH( $N=5)$} \\
\hline Age (years) & $64 \pm 9.65$ \\
\hline Gender (female) $(\mathrm{N})$ & 4 \\
\hline weight $(\mathrm{kg})$ & $85.4 \pm 19.75$ \\
\hline height(m) & $1.72 \pm 0.09$ \\
\hline \multicolumn{2}{|l|}{ Rats-Baseline ( $N=5$ ) } \\
\hline weight (kg) & $0.17 \pm 0.03$ \\
\hline \multicolumn{2}{|l|}{ Rats-Follow up $(N=5)$} \\
\hline weight (kg) & $0.23 \pm 0.02$ \\
\hline
\end{tabular}

\subsubsection{Methods}

Each image was manually processed using our software implementation without automation. The spline control points were adjusted to achieve optimal ROI fitting, and the results were judged by the same operator for model appropriateness using visual inspection of the short axis (SA) and horizontal long axis (HLA) views. Thus the ability of the model to register each $\mathrm{RV}$ anatomy was evaluated.

The second part of the validation was to determine the effect of each control point and associated degrees of freedom in the fitting process, allowing the elimination of redundant control points and in some cases fixing control points prior to automatic fitting and optimization. For intersection points, the operator recorded the deviation from the initial estimate for each angle of each intersection point. All images that passed the 
appropriateness of the model validation were then used to evaluate the performance of automatic fitting.

\subsubsection{Results}

Of the 20 images analyzed, 14 passed the manual validation test. All human images were be accurately tracked by the spline model. All baseline rat images failed the test and 4 of 5 follow-up rat images passed the validation as summarized in Table 4-2. Example images are shown in Table 4-3 with a pass indicated by $\checkmark$ and a fail by $x$.

Most failure cases were due to limited visibility of the RV in the image. In most baseline rats the septum could not be delineated from the free wall due to small heart relative to the image spatial resolutions. Follow-up rats tended to have much larger hearts because of induced hypertrophy [50] and therefore the RV free-wall could be clearly delineated. In all human studies, the RV could be clearly visualized.

Table 4-2 - Summary of results per population category for manual fitting ( $N=20)$

\begin{tabular}{|c|c|c|c|}
\hline Group & Image Type & Passed & Failed \\
\hline \multirow{2}{*}{ Human } & Normal & 5 & 0 \\
\cline { 2 - 4 } & Hypertrophic & 5 & 0 \\
\hline \multirow{2}{*}{ Rats } & Baseline & 0 & 5 \\
\cline { 2 - 4 } & Follow-up & 4 & 1 \\
\hline
\end{tabular}


Table 4-3 - Sample results from validation of manually fitted results $(\checkmark=$ pass,$x=$ fail)

\begin{tabular}{|c|c|c|c|}
\hline \multirow{2}{*}{ Specimen } & \multicolumn{2}{|l|}{ View } & \multirow{2}{*}{ Result } \\
\hline & HLA & $\mathbf{S A}$ & \\
\hline \multicolumn{4}{|l|}{ Human - Normal } \\
\hline \multicolumn{4}{|l|}{ Human - non-PH } \\
\hline \multicolumn{4}{|l|}{ Human - non-PH } \\
\hline \multicolumn{4}{|l|}{ Human - PH } \\
\hline Rat - Baseline & & & $x$ \\
\hline Rat - Follow-up & & & $\checkmark$ \\
\hline Rat - Follow-up & & & $x$ \\
\hline
\end{tabular}


From visual inspection of the control points, it was identified that the RV extent control point (point 11 in Figure 4-1) remained roughly medially situated between the septum and free wall. While point 11 was necessary to adjust the extent of the RV along the $z$-direction, the second degree of freedom, allowing the point to move in the $\mathrm{x}$ direction, was redundant. The model was therefore, simplified by fixing the $\mathrm{x}$ position of the extent point between the free-wall and septum.

A general trend was noted in the adjustment of anterior and posterior angles of intersection points (1/6 and 2/8 respectively in Figure 4.2). For each image that passed manual validation, the anterior angle need to be reduced from in the initial value of $140^{\circ}$, while the posterior angles needed to be increased from initial value of $220^{\circ}$. This trend in adjustment of angles indicated an overestimation and underestimation of the initial values of the anterior and posterior angles respectively for both atrial and mid-cavity planes.

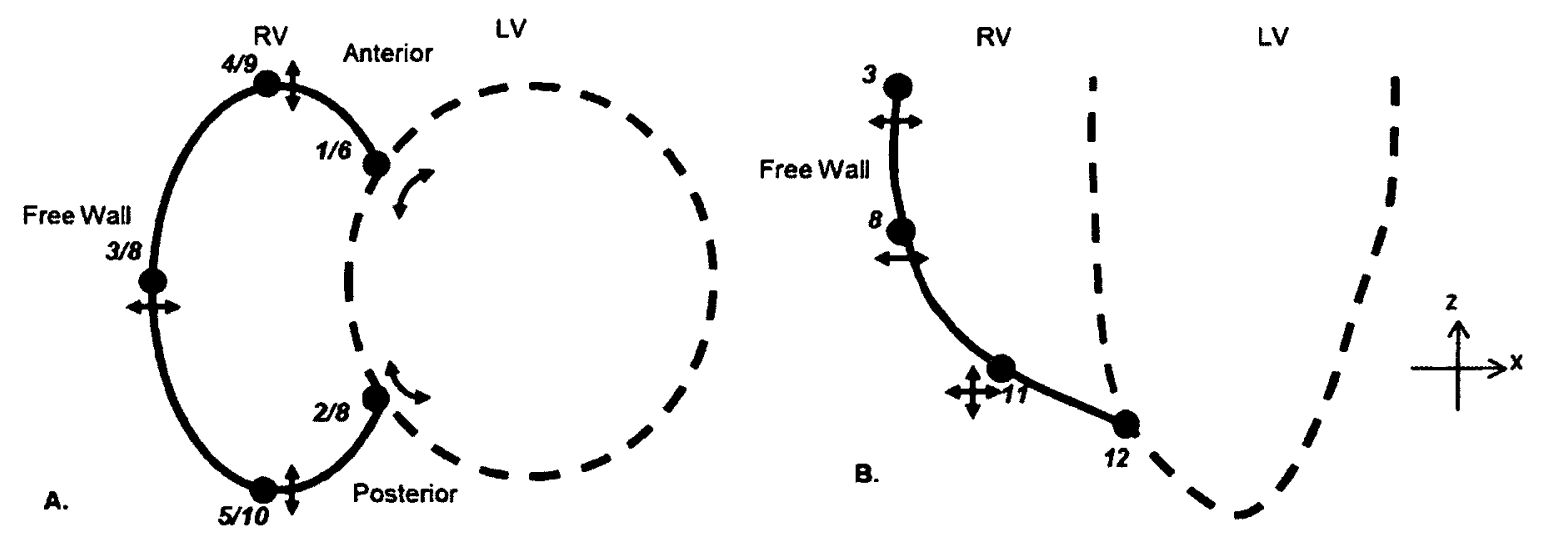

Figure 4-1: Reference diagram for control points as used in the validation

To improve initial estimates, all new values for angles after manual adjustments were recorded in Table 4-4 and then used to compute the average for both anterior and 
posterior angles. These average values were adopted as new initial estimates. A record of N/A signifies that a reading could not be reliable measured due to poor image quality. The average posterior angle was $245 \pm 5$ degrees and the average anterior angle was 120 \pm 10 degrees.

Table 4-4: Results from adjustment of intersection control points

\begin{tabular}{|c|c|c|c|c|c|}
\hline $\begin{array}{l}\text { Population } \\
\text { Type }\end{array}$ & Image & $\begin{array}{l}\text { Base } \\
\text { Posterior }\end{array}$ & $\begin{array}{l}\text { Base } \\
\text { Anterior }\end{array}$ & $\begin{array}{l}\text { Atrium } \\
\text { Posterior }\end{array}$ & Atrium Anterior \\
\hline \multirow{5}{*}{$\begin{array}{l}\text { Human non- } \\
\text { PH }\end{array}$} & 1 & 242 & 120 & 220 & 140 \\
\hline & 2 & 245 & 117 & 220 & 125 \\
\hline & 3 & 244 & 135 & 220 & 140 \\
\hline & 4 & 241 & 123 & 220 & 112 \\
\hline & 5 & 251 & 122 & 220 & 220 \\
\hline \multirow{5}{*}{ Human PH } & 1 & 243 & 99.5 & 220 & 124 \\
\hline & 2 & 235 & 125 & 220 & 140 \\
\hline & 3 & 245 & 124 & 220 & 140 \\
\hline & 4 & 252 & 129 & 220 & 140 \\
\hline & 5 & 254 & 94 & 220 & 140 \\
\hline \multirow{5}{*}{ Rat Baseline } & Rat 1 & $\mathrm{n} / \mathrm{a}$ & $\mathrm{n} / \mathrm{a}$ & $n / a$ & $n / a$ \\
\hline & Rat 2 & $n / a$ & $n / a$ & $n / a$ & $n / a$ \\
\hline & Rat 3 & 238 & 128 & 220 & 140 \\
\hline & Rat 4 & $n / a$ & $n / a$ & $\mathrm{n} / \mathrm{a}$ & $n / a$ \\
\hline & Rat 5 & $n / a$ & $n / a$ & $n / a$ & $n / a$ \\
\hline \multirow{5}{*}{$\begin{array}{l}\text { Rat } \\
\text { Follow up }\end{array}$} & Rat 1 & 240 & 117 & 220 & 140 \\
\hline & Rat 2 & $n / a$ & $\mathrm{n} / \mathrm{a}$ & $\mathrm{n} / \mathrm{a}$ & $n / a$ \\
\hline & Rat 3 & 247 & 124 & 220 & 140 \\
\hline & Rat 4 & 243 & 126 & 220 & 140 \\
\hline & Rat 5 & 246 & 118 & 220 & 140 \\
\hline \multicolumn{2}{|c|}{ Mean } & 245 & 120 & 220 & 142 \\
\hline \multicolumn{2}{|c|}{ Standard Deviation } & 5 & 10 & 0 & 23 \\
\hline
\end{tabular}

The remaining points were demonstrated to be critical to fitting the model to the range of images and varied between images. Points to be automatically optimized and their associated degrees of freedom are summarized in Table 4-5. Control points with 0 degrees of freedom indicate that they are not subjected to automatic optimization. These 
fixed points were kept as manual control points, however, the operator could use them to interact with the model if need be so as to be able to accommodate unforeseen physiologies which may not be represented by this sample image set.

Table 4-5: Modified Control Points and their degrees of freedom and initial location relative to the LV Long Axis

\begin{tabular}{|c|c|c|c|c|}
\hline $\begin{array}{c}\text { Control } \\
\text { Point }\end{array}$ & $\begin{array}{l}\text { Manual } \\
\text { Degrees of } \\
\text { Freedom }\end{array}$ & $\begin{array}{l}\text { Optimization } \\
\text { Degrees of } \\
\text { Freedom }\end{array}$ & Description & Initial Estimate \\
\hline 1 & 1 & 0 & $\begin{array}{l}\text { Basal Anterior RV/LV intersect } \\
\text { control point }\end{array}$ & $\begin{array}{l}\beta_{\mathrm{AB}}=120^{\circ} \text { around } \\
\mathrm{LVLA}\end{array}$ \\
\hline 2 & 1 & 0 & $\begin{array}{l}\text { Basal Posterior } \mathrm{RV} / \mathrm{LV} \text { intersect } \\
\text { control point }\end{array}$ & $\begin{array}{l}\beta_{P B}=240^{\circ} \text { around } \\
L V L A\end{array}$ \\
\hline 3 & 1 & 1 & $\begin{array}{l}\text { Basal Free wall spline points } \\
\text { RV/LV }\end{array}$ & $R_{F B}=2 \times b$ from LVLA \\
\hline 4 & 1 & 1 & $\begin{array}{l}\text { Basal Anterior vertical control } \\
\text { point }\end{array}$ & $R_{A B}=b$ from RVLA \\
\hline 5 & 1 & 1 & $\begin{array}{l}\text { Basal Posterior vertical control } \\
\text { point }\end{array}$ & $R_{P B}=b$ from RVLA \\
\hline 6 & 1 & 0 & $\begin{array}{l}\text { Mid Anterior } \mathrm{RV} / \mathrm{LV} \text { intersect } \\
\text { control point }\end{array}$ & $\begin{array}{l}\beta_{\mathrm{AM}}=120^{\circ} \text { around } \\
\mathrm{LVLA}\end{array}$ \\
\hline 7 & 1 & 0 & $\begin{array}{l}\text { Mid Posterior } \mathrm{RV} / \mathrm{LV} \text { intersect } \\
\text { control point }\end{array}$ & $\begin{array}{l}\beta_{\mathrm{PM}}=240^{\circ} \text { around } \\
\text { LVLA }\end{array}$ \\
\hline 8 & 1 & 1 & $\begin{array}{l}\text { Mid Free wall spline points } \\
\text { RV/LV }\end{array}$ & $R_{F M}=2 \times b$ from LVLA \\
\hline 9 & 1 & 1 & $\begin{array}{l}\text { Mid Anterior vertical control } \\
\text { point }\end{array}$ & $R_{A M}=b$ from RVLA \\
\hline 10 & 1 & 1 & $\begin{array}{l}\text { Mid Posterior vertical control } \\
\text { point }\end{array}$ & $R_{P M}=b$ from RVLA \\
\hline 11 & 2 & $\begin{array}{l}1 \text { (longitudinally } \\
\text { only) }\end{array}$ & $\begin{array}{l}\text { RV Extent spline point -useful } \\
\text { particularly in hypertrophic case } \\
\text { were the RV shape requires more } \\
\text { flexibility to trace out. }\end{array}$ & $\begin{array}{l}\text { Midway between the } \\
\text { RV/LV intersect control } \\
\text { point and } z=0\end{array}$ \\
\hline 12 & 1 & 1 & $\mathrm{RV} / \mathrm{LV}$ intersect control point & $\theta=90^{\circ}$ \\
\hline
\end{tabular}

\subsection{Validation of Automatic Fitting and Optimization}

The revised model (with reduced degrees of freedom and updated initial estimates) was evaluated for automatic fitting performance, an iterative process that adjusts control points based on the image intensity and assumed shape of the RV. Automatic fitting and optimization was applied to all the images that passed manual 
validation (14 in total). These sets of images included 5 non PH patients, 5 PH patients and 4 follow up rats. Automation looked into three different aspects of the image; (1) the ability to automatically register the entire $R V$, (2) the ability to automatically fit $R V$ apex to the RV valve plane region and (3) the ability to automatically fit the RV free wall correctly (region visible in HLA display). During validation for automatic fitting, the operator aided the software in the reorientation phase by ensuring accurate ellipse placement but did not intervene during the registration phase. The operator relied on visual inspection to determine if the region of interest was correctly defined by the process. The operator observed the model fit in both short axis (SA) and horizontal long axis (HLA) views and graded pass " $\checkmark$ " for a correct fit and fail " $x$ " otherwise, as indicated in the sample results in Table 4-6. 


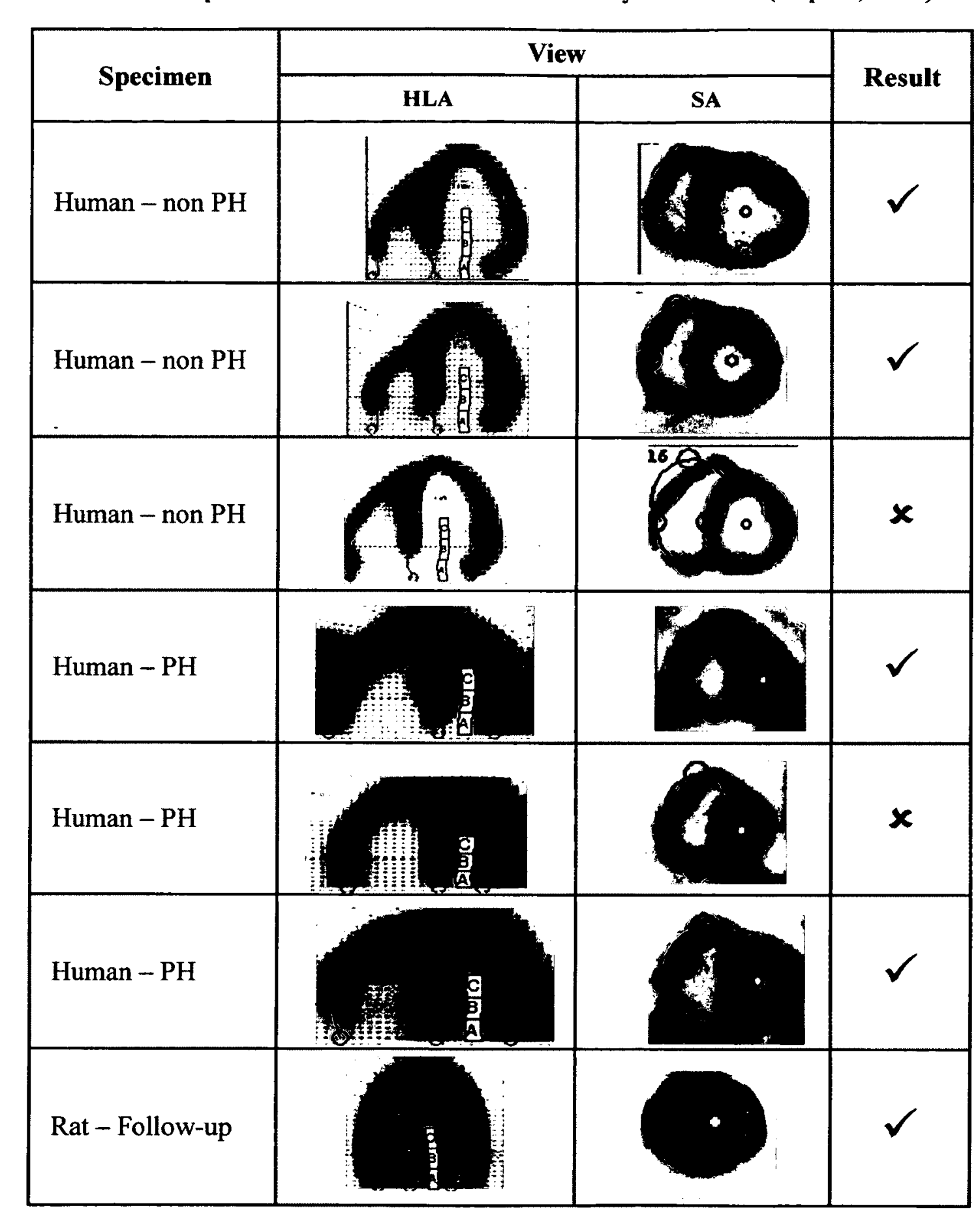




\subsubsection{Results}

As indicted in Table 4-7, out of 14 images validated for automatic fitting; 8 passed the validation for global RV fitting, 7 passed validation for RV fitting from apex to midsection and 13 passed validation for RV free wall fitting. Table 4-7 shows the complete break down for the automatic fitting results in different populations.

Table 4-7 : Validation Results for automatic fitting for 3 regions of the RV( $\checkmark=p a s s, x=$ fail $)$

\begin{tabular}{|c|c|c|c|c|}
\hline $\begin{array}{l}\text { Population } \\
\text { Type }\end{array}$ & Specimen & $\begin{array}{l}\text { Complete } \\
\text { Automation } \\
\text { (Includes all control } \\
\text { points) }\end{array}$ & $\begin{array}{l}\text { Apex to } \\
\text { Midsection } \\
\text { (all base control } \\
\text { points } 8,11,12 \text { ) }\end{array}$ & $\begin{array}{l}\text { Free wall } \\
\text { (control points } 3 \text {, } \\
8,11 \text {, and } 12 \text { ) }\end{array}$ \\
\hline \multirow{5}{*}{ Human non PH } & 1 & $\checkmark$ & $\checkmark$ & $\checkmark$ \\
\hline & 2 & $x$ & $x$ & $\checkmark$ \\
\hline & 3 & $x$ & $x$ & $\checkmark$ \\
\hline & 4 & $\checkmark$ & $\checkmark$ & $\checkmark$ \\
\hline & 5 & $\checkmark$ & $\checkmark$ & $\checkmark$ \\
\hline \multirow{5}{*}{ Human PH } & 1 & $\checkmark$ & $\checkmark$ & $\checkmark$ \\
\hline & 2 & $x$ & $\checkmark$ & $\checkmark$ \\
\hline & 3 & $x$ & $\checkmark$ & $\checkmark$ \\
\hline & 4 & $\checkmark$ & $\checkmark$ & $\checkmark$ \\
\hline & 5 & $x$ & $\checkmark$ & $\checkmark$ \\
\hline \multirow{4}{*}{ Rat Follow up } & Rat 1 & $\checkmark$ & $\checkmark$ & $\checkmark$ \\
\hline & Rat 3 & $\checkmark$ & $\checkmark$ & $\checkmark$ \\
\hline & Rat 4 & $x$ & $x$ & $\boldsymbol{x}$ \\
\hline & Rat 5 & $\checkmark$ & $\checkmark$ & $\checkmark$ \\
\hline
\end{tabular}


Automatic fitting performs well in the free wall section in $13 / 14$ cases as indicated in Table 4-7, this can be attributed to the fact that the free wall is well pronounced. Apex to midsection regions exhibited more failures, partly due to spillover from the LV. The extent control point required more adjustment to correctly map the shape of the RV particularly in the apical region. These results highlight regions of the RV ROI that needs more attention for enhancing the fitting algorithm in order to achieve better overall automation.

Diminished RV image intensities, especially around the atrial region as observed in some of the images in Table 4-6, also resulted in suboptimal optimization. Automation was successful in all follow-up cases for rats used in except Rat 4, which appeared to have not developed severe hypertrophy with MCT treatment. It was also observed that visualization of the right atrium (RA) was nearly impossible in most rat images where is the myocardial wall is even thinner.

Successful definition of RV ROIs is also depends on the successful LV reorientation and registration. Because initial estimation of control points for RV registration is based on the shape of $\mathrm{LV}$, poor definition of the LV ROI may result in poor estimates of the control points. Lastly, regions near the RV/LV intersection are susceptible to spillover artifacts from the LV resulting in the optimization algorithm skewing the RV model towards the LV.

\subsection{Operator variability}

A separate set of images consisting of 19 images (7 subjects with confirmed $\mathrm{PH}$ and 12 arbitrarily selected patients referred for clinical viability studies using FDG-PET) 
was used to evaluate operator variability. The population details are summarized in the Table 4-8.

Table 4-8 : Population characteristics for subjects for operator variability analysis

\begin{tabular}{ll}
$\mathbf{N}=19$ & Mean \pm SD \\
\hline Age (years) & $65 \pm 10$ \\
Gender (female) $(\mathbb{N})$ & $\mathbf{8}$ \\
weight $(\mathrm{kg})$ & $\mathbf{8 8 . 5} \pm 14.46$ \\
height $(\mathrm{m})$ & $1.71 \pm 0.08$ \\
confirmed PH & 7 \\
Non- $\mathrm{PH}$ & 12 \\
\hline
\end{tabular}

Each of two operators processed and analyzed the entire set of images twice from the reorientation stage through to the registration phase. The operator manually adjusted control points in cases where automatic process produced a poor fit. During processing the operator monitored the number of interventions and control points adjusted. Operator 1 was experienced with the RV registration software, while operator 2 was familiar with FlowQuant ${ }^{\mathfrak{O}}$, but not with the RV sampling GUI. Both operators processed each dataset twice in random order and on separate days so as to reduce memorization biases.

\subsubsection{Statistical Analysis}

The reprocessed datasets were analyzed for inter and intra-operator variability in relative uptake (each polar map normalized to the $95^{\text {th }}$ percentile highest pixel) for 3 vascular regions, namely the distal, medial and proximal RV free wall (Figure 3-13).

The region around anterior and posterior RV/LV incision points was trimmed from the hemi-polar map as well as the atrium region (denoted as trimmed region in Figure 3-13). Each region was analyzed using the following methods: 
a. Linear regression analysis - determining correlation between percentage uptake values for (a) two datasets from for each operator (intra-operator) (b) the two operators' datasets (inter-operator). The level of agreement between datasets was report by Pearson's correlation coefficient $\left(r^{2}\right)$, with $r^{2}=1$ representing perfect agreement.

b. Bland-Altman analysis - determining the difference between paired datasets by generating Bland-Altman plot [6] and evaluating coefficient of reproducibility (RPC). The RPC value was calculated as the standard deviation of the differences between paired datasets multiplied by $1.96[6,45,46]$. The $95 \%$ confidence limit of agreement was evaluated as the mean $\pm \mathrm{RPC}$ assuming the measurements are normally distributed. \%RPC is the percentage ratio of the $\mathrm{RPC}$ value to the mean of uptake values.[6] The lower the RPC value the more reproducible the results are.[45, 47] To assess if there any systematic differences between paired datasets a t-test was used with $p<0.05$ considered significant $[6,45,47]$.

c. Sample point variability analysis - determining how much the location of sample points varied between two tests for each operator and between operators. For each region, the Euclidean distance was measured between each sample point's locations in repeated measurements (i.e. inter- and intra-operator position variability). A hemi polar map was generated using distance differences of each sample point, and segmental averages were also reported. The variation of mean distances between sample point positions was also reported using histograms. 


\subsubsection{Results}

Table 4-9 lists the number of user interventions recorded for each control point during each run. Operators consistently intervened more frequently at apical (apex and extent) and anterior/posterior extent regions, indicating that automation in the free wall regions was judged to be more adequate. Relatively few interactions with the anterior/posterior intersect control point were logged, indicating appropriate fixing of the intersection angles. Overall the second operator (inexperienced) intervened more (often and number of control points in each image) than the first operator in both test cases.

Table 4-9 : Number of interventions during operator variability testing.

\begin{tabular}{|c|c|c|c|c|c|c|c|c|c|c|}
\hline \multirow{2}{*}{ Op. } & \multirow{2}{*}{ Test } & \multicolumn{8}{|c|}{ Number of interventions } \\
\cline { 3 - 12 } & Apex & Extent & $\begin{array}{c}\text { Basal } \\
\text { FW }\end{array}$ & $\begin{array}{c}\text { Atrial } \\
\text { FW }\end{array}$ & Ant.* & Post.* & $\begin{array}{c}\text { Ant } \\
\text { int.* }\end{array}$ & $\begin{array}{c}\text { Post. } \\
\text { Int* }\end{array}$ & Total \\
\hline \multirow{2}{*}{1} & $\mathbf{1}$ & 4 & 6 & 2 & 2 & 9 & 3 & 2 & 2 & 30 \\
& 2 & 6 & 5 & 2 & 3 & 8 & 3 & 4 & 4 & 35 \\
\hline \multirow{2}{*}{2} & $\mathbf{1}$ & 7 & 9 & 3 & 4 & 8 & 3 & 4 & 1 & 39 \\
& $\mathbf{2}$ & 8 & 10 & 2 & 2 & 9 & 2 & 6 & 3 & 42 \\
\hline \multicolumn{2}{|c|}{ TOTAL } & 25 & 30 & 9 & 11 & 34 & 11 & 16 & 10 & 146 \\
\hline
\end{tabular}

FW $=$ Fee Wall int. - intersection

Post. - Posterior Ant. - Anterior

* Column accounts for interventions at both the base and the atrium

\subsubsection{Intra-Operator Variability}

The hemi-polar maps in Figure 4-2 indicate the mean distances (Euclidean distance) between the two locations of sampling points for each operator's datasets. The mean distance increased towards the apex consistently for both operators. As shown in

Table 4-9 apical section which forms part of the distal region recorded highest number of user interventions. Histograms of means distances between sampling points (Figure 4-3) confirmed these results, and recorded the highest mean distances in the distal region (up to $15 \mathrm{~mm}$ ) for both operators. This indicates that the automatic processing 
performed poorly and operator intervention was not reliable in this region. Most of the challenges were attributed to the wide range of morphologies of the RV, which complicates the automatic registration process by demanding intricate and increased number of constraints. The proximity of the RV apex to the more pronounced LV in this region can in part lead to poor delineation of the RV and consequently poor performance of the fitting algorithm as well as impaired operator judgment. The global RV free wall, a part of proximal region (Figure 3-13), recorded the lowest mean distances between sampling points for both operators. This supports previous validation of automatic results, which indicated that automatic registration (Table 4-7) of the RV free wall was successful more than $90 \%$ of the time. The histograms of mean distances indicate low variability in the sample point placement with about $95 \%$ of sample points having less than $5 \mathrm{~mm}$ differences for both operators.
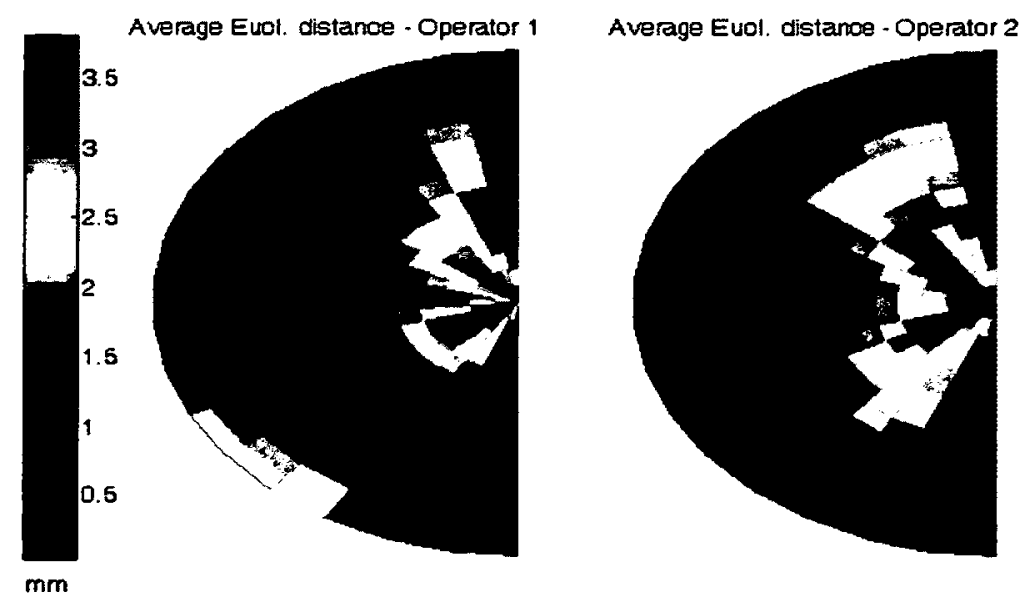

Figure 4-2 : Hemi polar map of mean distances between sampling points in the paired datasets from for operator 1 and operator 2. 


\section{Operator I}
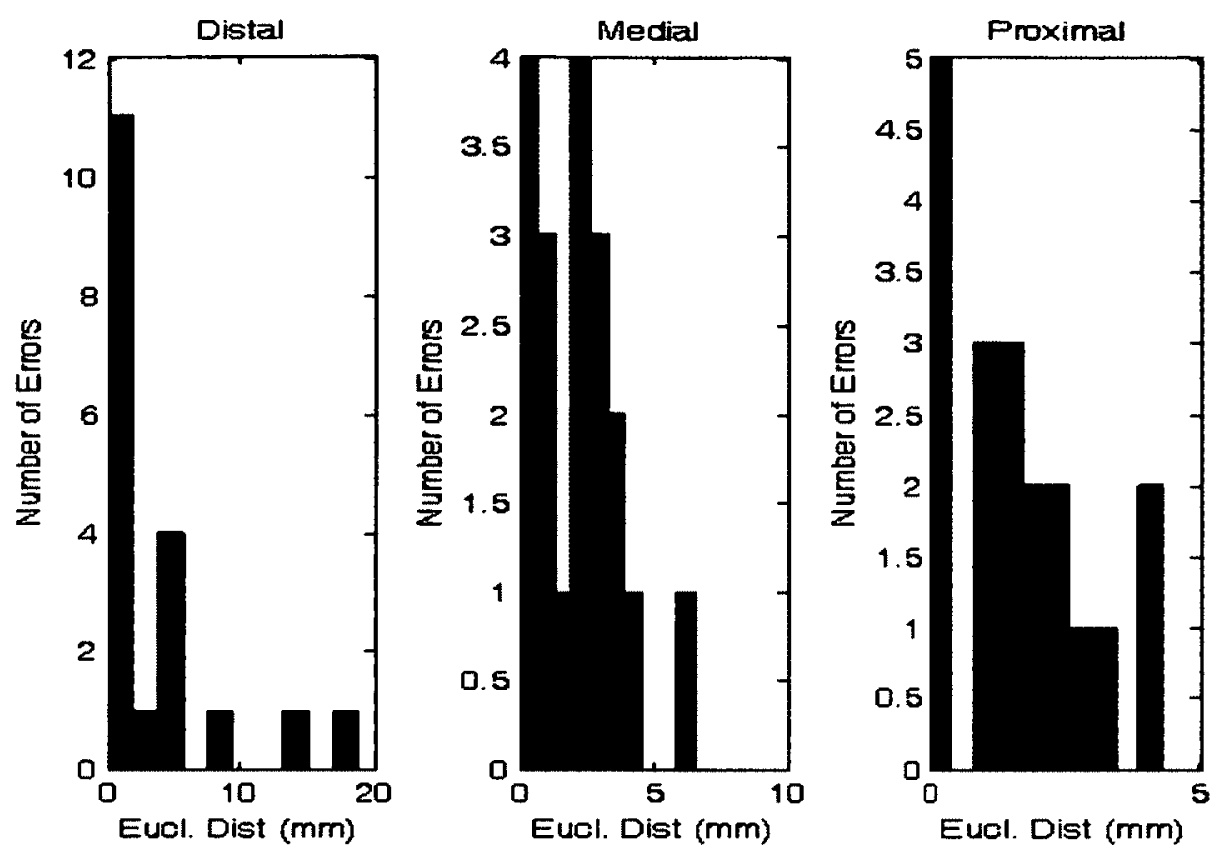

\section{Operator II}
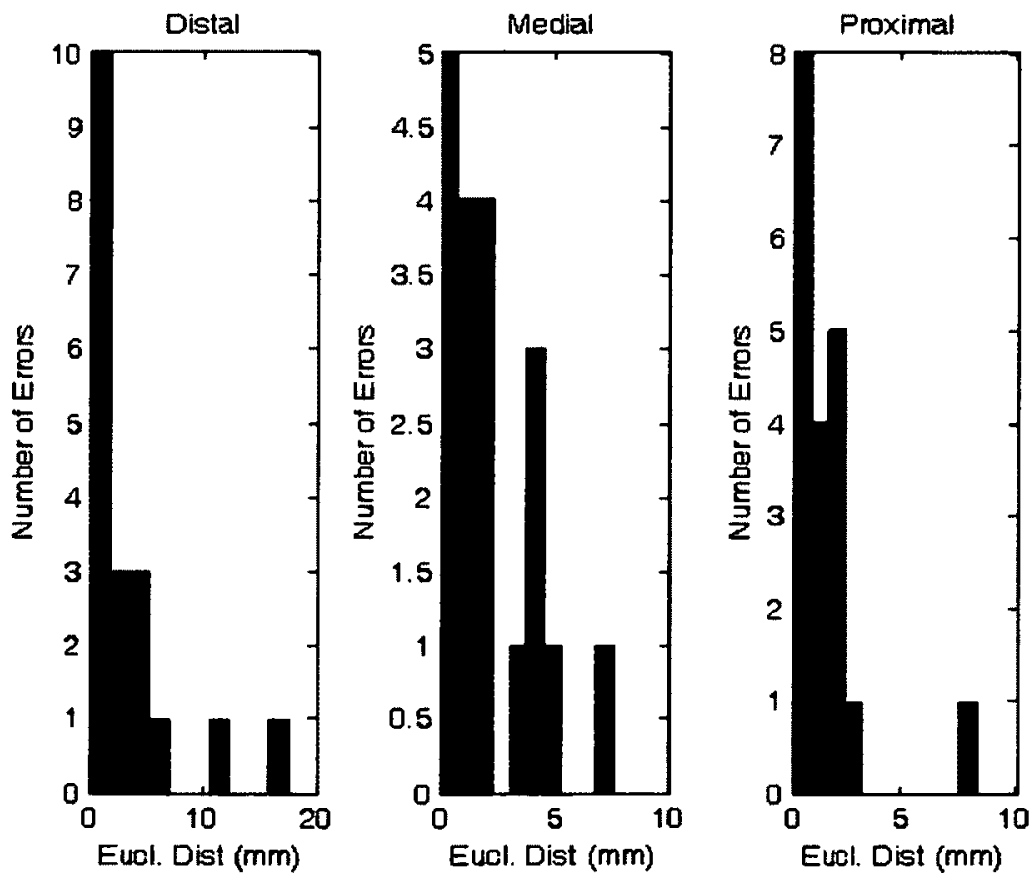

Figure 4-3 : Histogram of means Euclidian distances for intra operator variability for operator 1 and operator 2. 
Linear regression analysis of percentage relative tracer uptake (Figure 4-4) for both operator 1 and operator 2 measured $r^{2}=0.959$ and 0.942 respectively, indicating excellent agreement between repeat analyses by the same operator. Slopes for both lines were close to unity with values 0.99 and 1.01 for operator 1 and operator 2 respectively, while absolute value of the $y$-intercept for both operators were less than $1 \%$ indicating no significant bias in relative tracer uptake between runs.

Bland-Altman analysis of intra operator variability for all regions combined $(n=57)$ for each operator as shown in Figure 4-4. Mean differences were not significantly different from zero $(\mathrm{p}=\mathrm{n} . \mathrm{s}$.) indicating no bias between test and retest by the same operator. The reproducibility coefficients were found to be $5.6 \%$ and $6.4 \%$ for operator 1 and operator 2 respectively, which is comparable with LV analysis tools implying that both users generated acceptable relative uptake measurement reproducibility for current standards in the field of nuclear cardiology [23]. 


\section{Intra-Operator 1 Uptake}
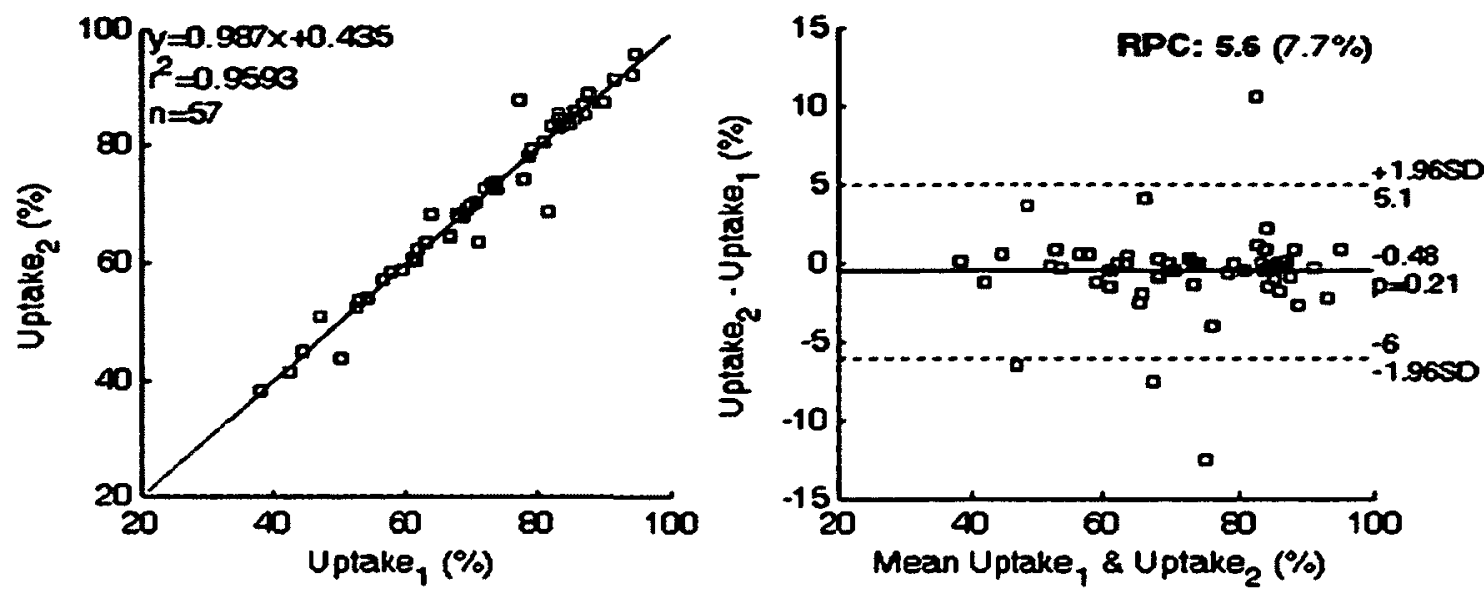

Intra-Operator 2 Uptake
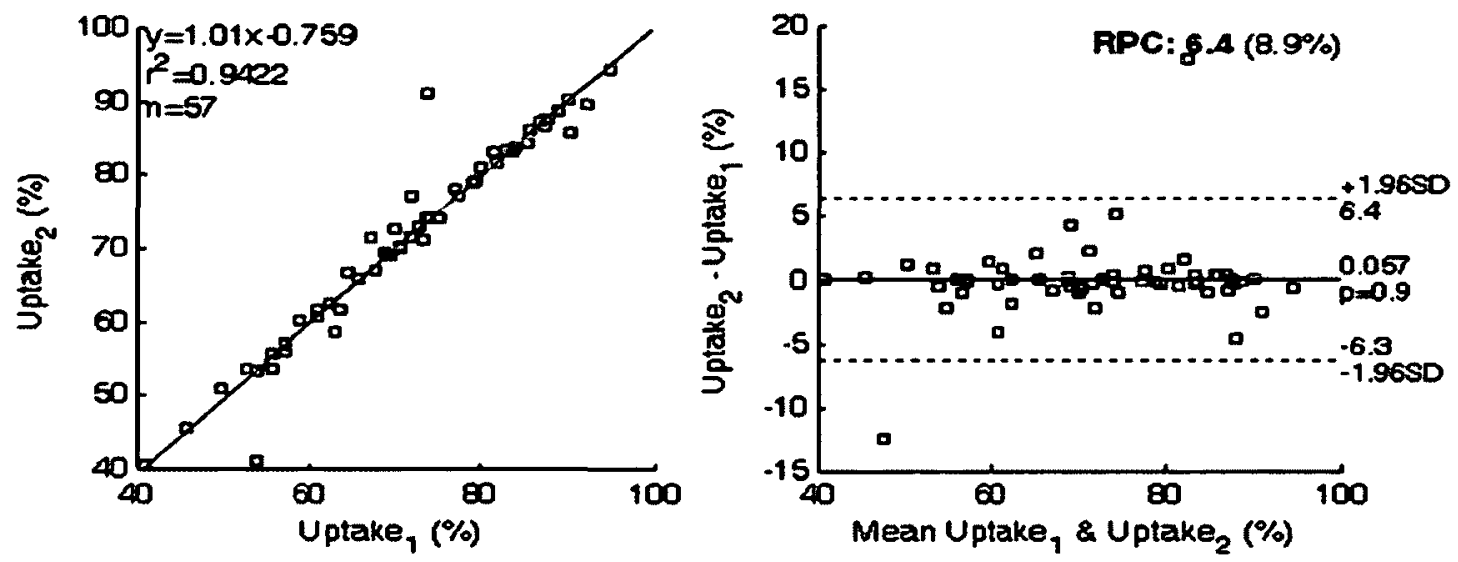

- oIstal

a

PROXIMAL

Figure 4-4 : Linear Regression plot and Bland Altman plot depicting results from intra-operator variability for operator 1 and operator 2 . 


\subsubsection{Inter Operator Variability}

The medial region had the smallest distance between sample points between operators, while larger variations were noticeable in the distal (apex) region as shown in Figure $4-5.85 \%$ of sample points mean distances were $\leq 5 \mathrm{~mm}$, across all regions (Figure 4-6). From Figure 4-6 there is a good correlation of relative tracer uptake between the two operators as indicated by the Pearson $r^{2}=0.910$ for both data sets and all territories $(n=114$ data points). The slope of the linear regression plot was 1.01 , which is close to unity and nearly zero $y$-intercept $(0.26 \%)$ indicating low bias [44].

Figure 4-6 illustrates the extent of inter operator variability as analyzed using Bland-Altman technique and the correlation between uptake values from two users. The mean difference between the uptake values, bias, is $0.28 \%$ which was not significantly different from $0(\mathrm{p}=\mathrm{ns})$. The $95 \%$ confidence interval for relative tracer uptake values is 7.9 to $8.5 \%$ which is consistent with previously reported LV analysis tools [6].

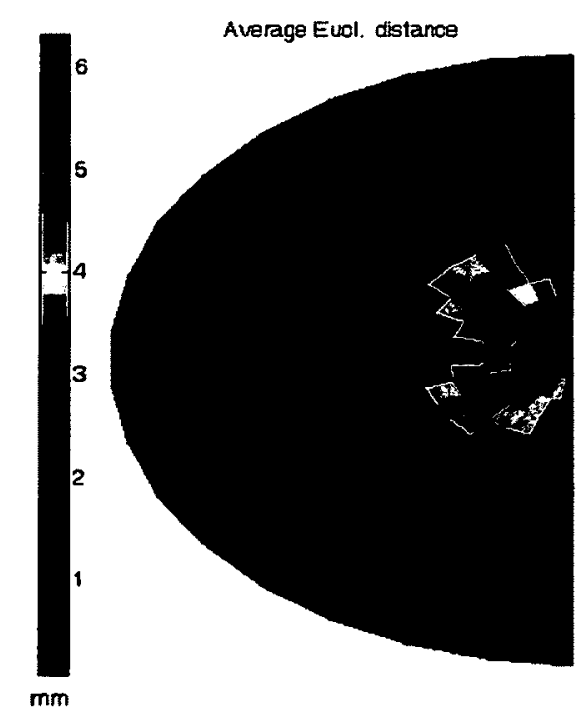

Figure 4-5 : Hemi polar showing mean Euclidian distance from inter operator variability 

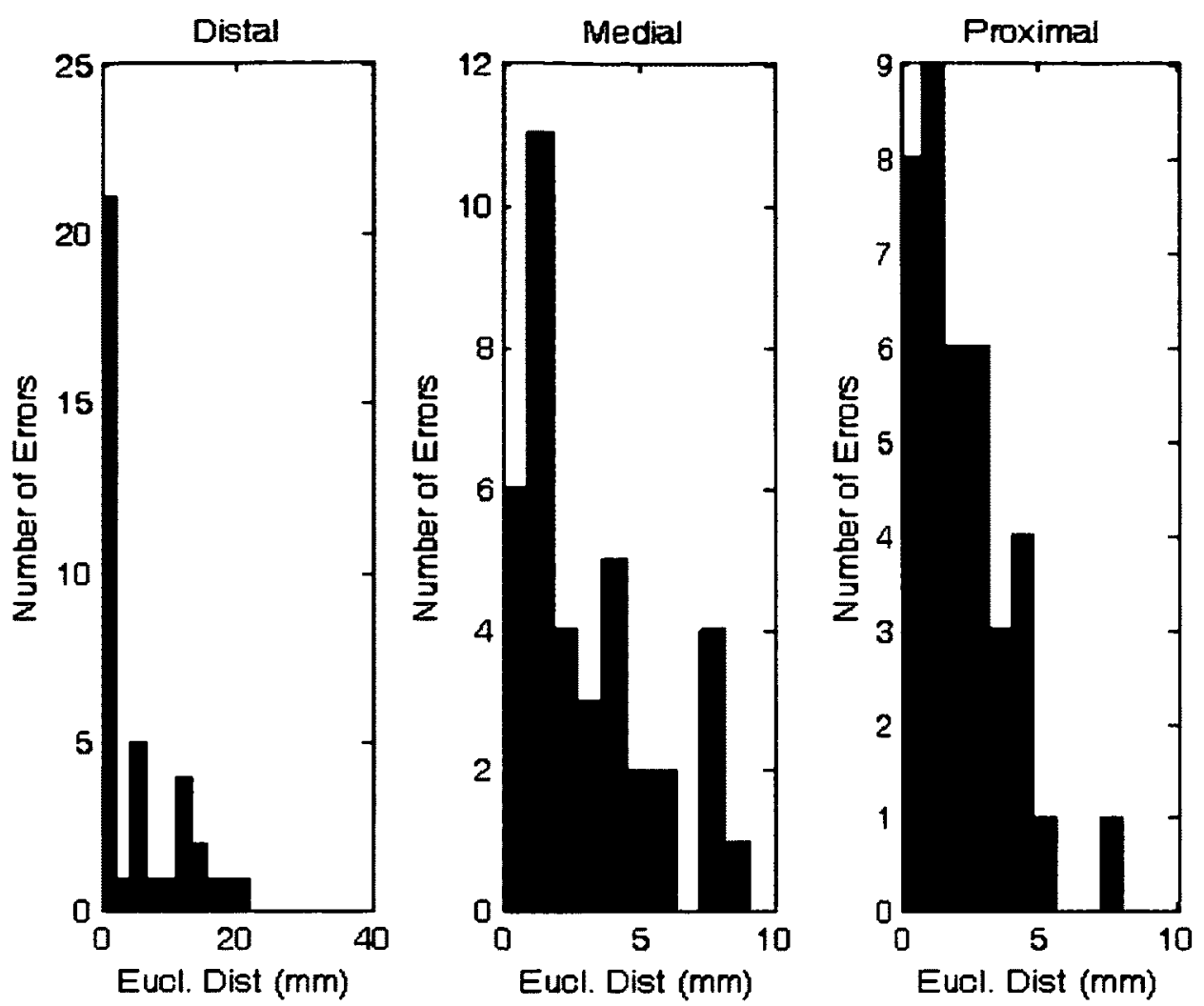

Figure 4-6 : Histogram of mean Euclidian distance (errors) for 3 vascular territories of the RV region on interest.

\section{Inter-Operator Uplake}
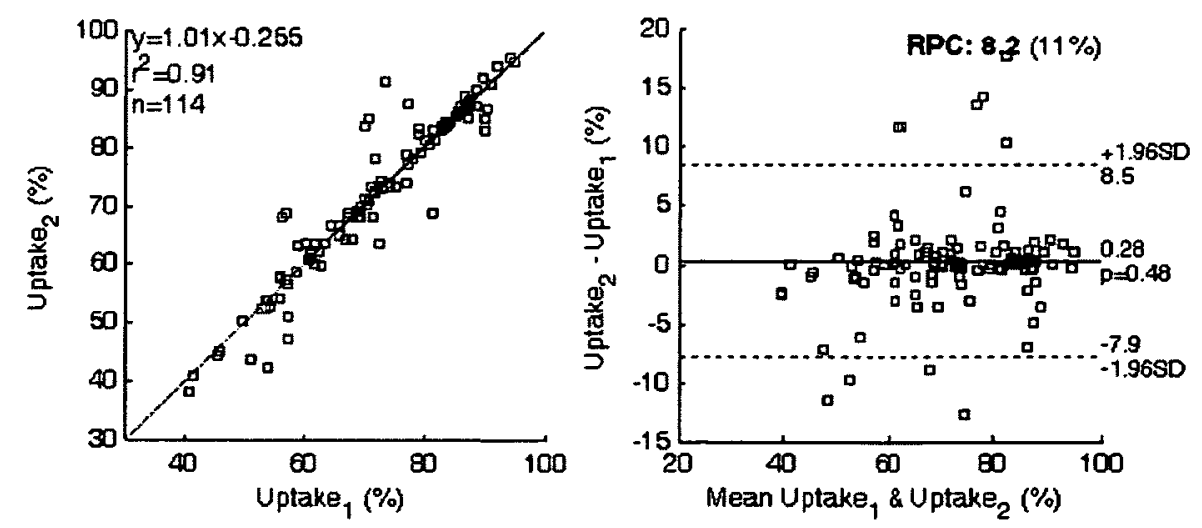

\begin{tabular}{llllll}
\hline D DISTAL & 0 & MEDIAL & D & PROXIMAL \\
\hline
\end{tabular}

Figure 4-7 : Linear Regression plot and Bland Altman plot depicting results for inter operator variability between operator 1 and operator 2 . 


\subsection{Validation of Cavity Volumes and Ejection Fractions}

The goal of validating cavity volumes and ejection fractions is to establish consistency and confidence in the results obtained. Cavity volumes and ejection fractions were compared against values reported using cardiac magnetic resonance imaging (CMR) in 5 confirmed PH patients.

Cavity volumes from CMR scans were measured using a clinically validated software Argus (Siemens, Erlangen, Germany) [55, 56]. PET scans for the patients were performed in the same week as CMR scans.

Two separate Cardiac PET images for each patient were acquired using $\left[{ }^{18}\right.$ F]fluoro-6-thia-heptadecanoic acid (FTHA) and 2-deoxy-2- $\left({ }^{18} \mathrm{~F}\right)$ fluoro-D-glucose (FDG) tracers which are both labeled using fluorine-18 $\left({ }^{18} \mathrm{~F}\right)$. In each patient a single image was selected for the analysis based on visual selection for best image quality. The operator intervened where deemed necessary to adjust the control points during gated analysis of cardiac PET images. During processing of the PET images, the user was blinded to the CMR results, and the comparison was conducted retrospectively, so as to avoid biasing the operator to CMR results. Furthermore the user was not privy to the CMR images, only the final cavity volumes and EF.

\subsubsection{Statistical analysis}

RV cavity volumes and EF from the current research (FlowQuant ${ }^{\mathbb{Q}}$ ) and Argus were reported as mean \pm SD .A further comparison between the measurements obtained acquired from PET and CMR scans were done using Bland-Altman agreement method. RPC represents the measurement accuracy compared to CMR reported values. The RPC 
value was calculated as the standard deviation of the differences between paired datasets multiplied by $1.96[45,46]$. The limit of agreement was evaluated as the mean \pm RPC assuming the measurements are normally distributed. \%RPC is the percentage ratio of the RPC value to the mean volume value for cavity volume.

\subsubsection{Results}

EDV, ESV and EF were measured to be $90 \pm 25 \mathrm{~mL}, 61 \pm 78 \mathrm{~mL}$ and $37 \pm 13 \%$ using PET and $88 \pm 17 \mathrm{~mL}, 56 \pm 23 \mathrm{~mL}$ and $33 \pm 7 \%$ using CMR as summarized in Table 4-10. Average cavity volumes and EF tended to be higher PET to CMR. From Figure 4-8, there is a good correlation of cavity volumes and EF between PET and CMR as indicated by the Pearson $r^{2}$ of 0.954 and 0.965 for cavity volume and ejection fraction respectively. The slope of the linear regression plot was 0.866 for cavity volume indicating cavity volume underestimation by PET and 1.1 for EF which is close to unity and y-intercepts for volumes and EF are 5.22 and -1.24 respectively. Lower PET volumes can be attributed to several factors including the difference in estimation of the location of basalvalve plane and inaccurate estimation of the $\mathrm{RV}$ myocardial wall thickness $[56,57]$. However, the Bland-Altman analysis (Figure 4-8) did not indicate that the volumes were biased. The mean difference between the cavity volumes, was $-3.4 \mathrm{~mL}$ which was not significantly different from $0(p=0.13)$. The $95 \%$ confidence interval for cavity volume values was -16 to $9 \mathrm{~mL}$ which has a narrower limits of agreement than previously reported by Grapsa et al $(-59.9$ to $52.5 \mathrm{~mL})$. Likewise both RPC values, $13(17 \%)$ and 6.3 (17\%) for cavity volumes and EF respectively suggests that PET measurement agreed 
with CMR measurements better than previously reported agreement between CMR and 3D-echocardiography, and similarly or better CMR operator variability.

Table 4-10: Mean \pm SD of cavity volumes and EF measurements acquired from PET and CMR cardiac images.

\begin{tabular}{|c|c|c|c|}
\hline Measurement & PET $(n=5)$ & CMR $(n=5)$ & p-value \\
\hline R-EDV (mL) & $90.4 \pm 25.2(82.8-119)$ & $\begin{array}{c}88.7 \pm 17.43(64- \\
110.2)\end{array}$ & n.s. \\
\hline R-ESV (mL) & $61 \pm 78(22.8-90)$ & $56.7 \pm 23.6(23-85.4)$ & n.s. \\
\hline R-EF (\%) & $37 \pm 13.2(28-57)$ & $33 \pm 7(18.5-49)$ & n.s \\
\hline
\end{tabular}

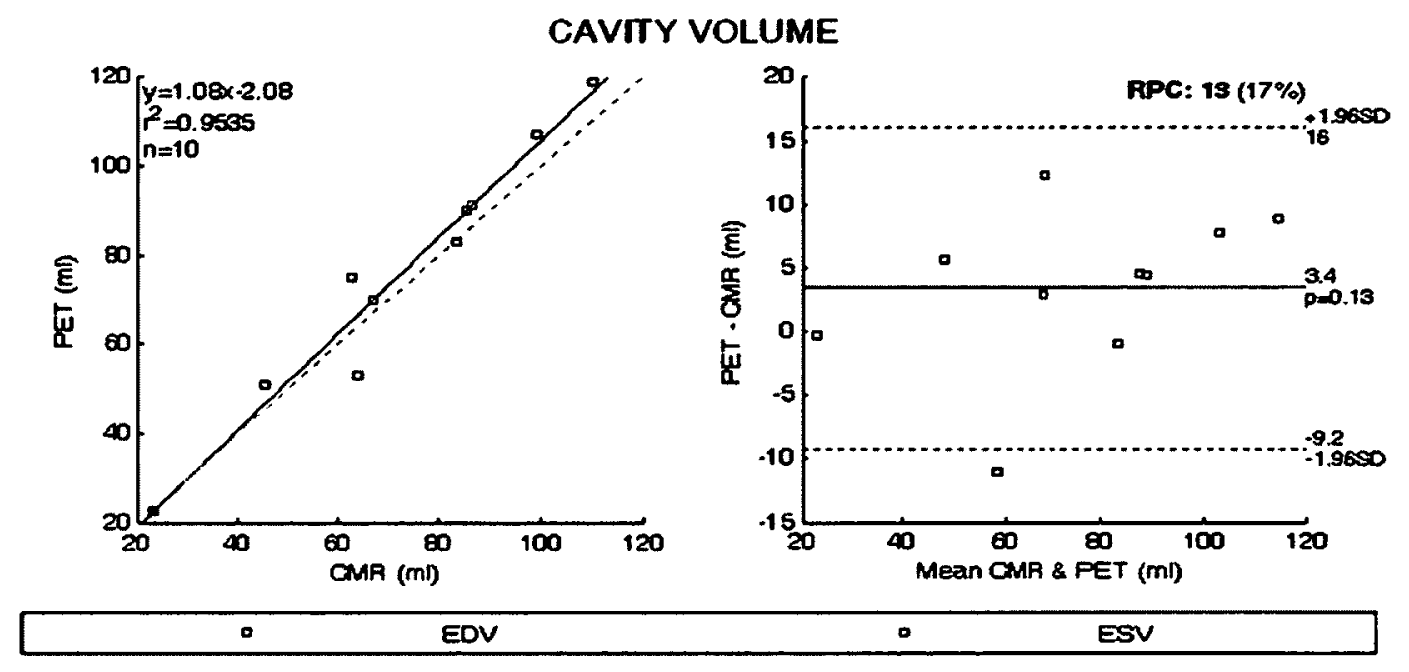

EF
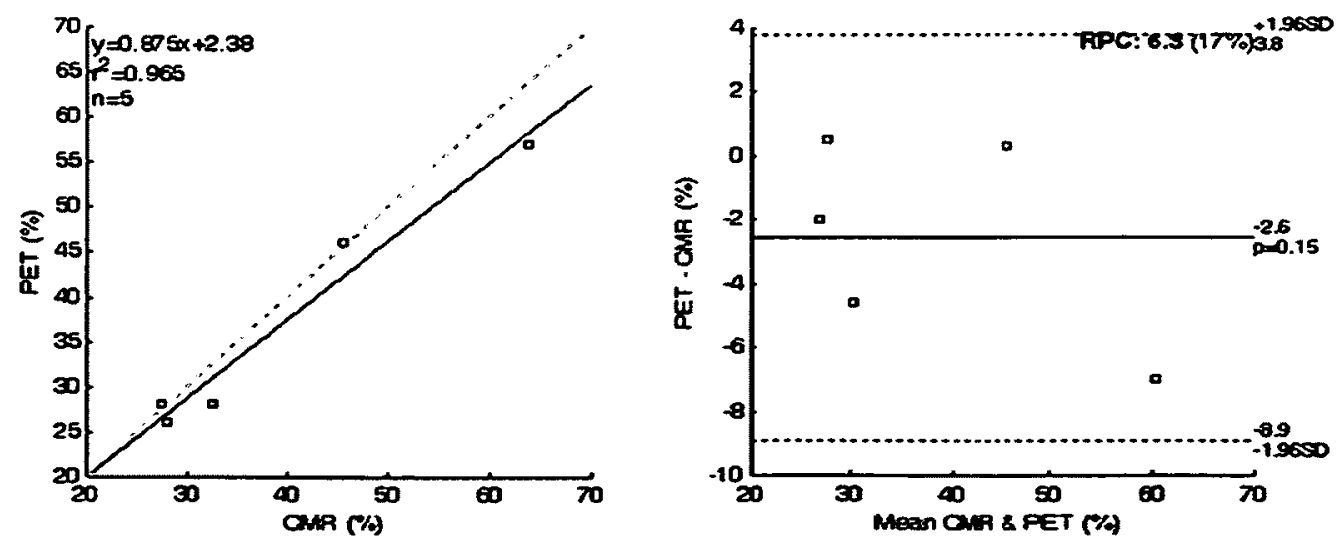

Figure 4-8 : Regression and Bland-Altman plot comparing cavity volumes and EF measurements acquired from PET and CMR cardiac images. 


\subsection{Conclusion}

We evaluated the adequacy of the model to accommodate a wide range of morphologies including normal and hypertrophic hearts in both rats and humans. Evaluation of automated fitting performance concluded that $50 \%$ of cases did require operator intervention. However, operator variability results indicated a good agreement between repeated measurements for each operator as well as between the two operators. From gated analysis results we demonstrated ability to reliably measure RV cavity volumes and EF as confirmed by a comparison with CMR results in human studies.

Low operator variability in both uptake and volume measurements indicate a tool that is useful for research in hypertrophic rat hearts and normal and hypertrophic human studies. 


\section{Chapter: Discussion}

In this research we designed and implemented an algorithm for semi-automatic registration of the RV. We established that RV function can be semi-automatically quantified using PET images in a wide range of morphologies associated with the presence and absence of RV hypertrophy. We established that the region of interest of the RV can be automatically segmented and used in both dynamic analysis and gated analysis, but that manual operator intervention is required in approximately $50 \%$ of the study cases. It is possible that RV ROI can be completely defined manually but that would require a lot of operator time. This utility drastically reduces operator time and results become more reproducible with the improvement of the automation process.

Despite the significant level of manual intervention, this research demonstrated that the registration algorithm can be used to analyze both normal and hypertrophic human hearts and hypertrophic rat hearts. The analysis tool had limited utility in normal rats due to insufficient image resolution for the small RV cavity size and anatomy.

Both inter- and intra-operator variability as measured by Pearson correlation coefficient, $r^{2}$, was excellent and comparable to previously published $r^{2}$ values [6] as listed in (Table 5-1). However RPC of relative uptake values were significantly higher than previously reported values for LV relative uptake as in Klein et al. While LV relative uptake PMs are normalized to the region of highest activity, RV PMs are normalized to the same LV, which may be a source of increased variability in RV measurements vs. LV. 
Table 5-1: Comparison of Pearson correlation coefficients $\left(r^{2}\right)$ and RPC $(\%)$ values between current work and work by Klein et al [23].

\begin{tabular}{|c|c|c|c|c|c|}
\hline \multicolumn{2}{|c|}{} & \multicolumn{2}{c|}{ Current Research } & \multicolumn{2}{c|}{ Klein et al } \\
\cline { 3 - 6 } \multicolumn{2}{|c|}{} & $r^{2}$ & RPC & $r^{2}$ & RPC \\
\hline \multirow{2}{*}{$\begin{array}{c}\text { Intra } \\
\text { Operator } \\
\text { Variability }\end{array}$} & $\begin{array}{c}\text { Op 1 } \\
\text { (expert) }\end{array}$ & 0.987 & 5.6 & 0.9771 & 0.97 \\
\cline { 2 - 6 } & $\begin{array}{c}\text { Op 2 } \\
\text { (novice) }\end{array}$ & 0.942 & 6.4 & 0.976 & 1.2 \\
\hline \multicolumn{2}{|c|}{ Inter Operator Variability } & 0.910 & 8.2 & 0.9771 & 1.8 \\
\hline
\end{tabular}

While complete automation of ROI registration was not obtained, the algorithm performed well in segmentation of the free wall as depicted by results in Table 4-7. The free wall is expected to have the greatest clinical significance in studying the RV functionality particularly, glucose metabolism in RV myocardium [51]. Furthermore, due to its increased distance from the LV septum, the free wall ROI is less vulnerable to spillover contamination from LV signals.

The ability to segment the RV ROI enables non-invasive assessment of RV function in in pathologies other than just pulmonary hypertension [51]. Of specific interest, therapies that regulate the demand of oxygen by the right ventricle can be evaluated and adjusted based on oxidative metabolic assessment of the RV [33, 51].

\subsection{Validation of Manual Fitting}

A wide range of images were used to validate the RV sampling model. This dataset demonstrated that the proposed model has sufficient degrees of freedom and control points to accurately define the RV ROI. Failure in the manual validation was primarily due to low RV image contrast resulting in difficulty in identifying the separation between the free wall and the septum as demonstrated in Figure 5-1. 
Visualization of the RV was prominent in rat images and primarily in base-line (normal) rats. The images that failed manual RV fitting were excluded from subsequent characterization of automatic RV registration.

A.

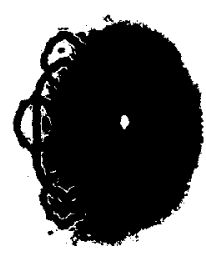

B.

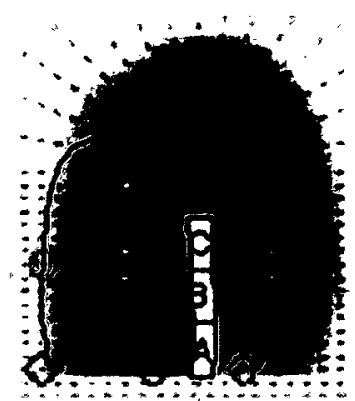

Figure 5-1 : A case of failed registration due to poor RV image intensity.(A) midsection SA slice (B) HLA slice. The RV free wall cannot be distinguished from the septum due to small feature size relative to image resolution, and low tracer uptake by the RV relative to the $L V$.

Monitoring of the manual fitting process established that there was a consistent tendency of the operator to adjust the anterior and posterior intersection points of the LV outwards increasing the breadth of the RV/LV intersection angle. To reduce this bias, the intersection points' angles initial estimates were set to an average value of the adjusted points in this data set. After improving their initial estimates, adjustments of intersection points were small enough for them to be fixed and enabling their removal from the automation process.

The extent control point which guides the shape of the RV at the apical region was also determined to have too many degrees of freedom. While initially it was moved both laterally (between septum and RV free wall) and vertically (parallel to the LV long axis), in the validation image set, it remained medially located between the septum and the free wall. These results supported simplification of the control point by restricting its 
motion to a single degree of freedom along the z-axis while fixing its lateral position mid-distance between the RV free wall and the septum.

The contours created from manual fitting give an approximate boundary of the region of interest but still required fine tuning for correct radial sampling of the mid myocardial wall (region with peak intensity). This flexibility proved adequate to accommodate virtually any RV morphology with only a limited set of model control points. Thus the automatic model fitting cost function was simplified to only 8 degrees of freedom as opposed to the initial 13 degrees of freedom.

\subsection{Validation of automatic fitting}

Fully automatic fitting was achieved in 8 of 14 test images. More operator intervention was observed in hypertrophic cases as shown in Table 4-7.

Automatic registration of the RV is challenging due to several factors:

1) RV image intensity: The intensity of the RV relative to the LV is lower primarily due to its much thinner wall and resulting partial volume effects consequent of limited spatial resolution. However, severe hypertrophy does exhibit comparable $\mathrm{RV}$ and LV image intensities due to increased wall thickening and reduced cardiac motion.

Diminished RV image intensities, especially approaching the atrial region as observed in some of the images in Table 4-7, also resulted in suboptimal optimization. Because of this problem, the actual shape of the RV near the atrium can only be assumed. It was also observed that the effect of visualization of the 
right atrium (RA) was nearly impossible in most rat images where is the image resolution is even more challenging.

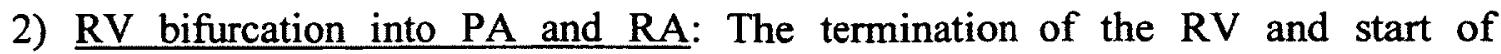
pulmonary artery (PA) or RA cannot be reliably determined using current imaging technology. The structural interference from the pulmonary trunk was not modeled in the automatic registration process, and therefore leads to inconsistent results for the atrial region. Due to the difficulty of reliably defining the RA ROI, and its poor definition with current imaging technology, it may be suitable to exclude the RA from the model due to limited clinical utility, and inclusion of the RA may adversely impact analysis of the RV. Such changes to the model were not part of this work, but may warrant further consideration with improvements in image quality.

3) Wide Range of RV morphologies: As previously discusses, a wide range of RV morphologies must be accommodated during automation. The variations in shape impose a challenge in defining tight constraints without biasing against extreme morphologies. Tight constraints can aid convergence of the fitting algorithm by rejecting improbable morphologies.

4) Susceptibility to spillover contamination: Due to spillover from the LV at RV/LV intersection, the fine tuning algorithm is influenced by proximal LV structures and is aggravated by elevated intensities in the septum as demonstrated in the boxed region in Figure 5-2, resulting in premature contour intersection. Consequently, only analysis in the RV free wall, distal to the LV, may be reliable for measuring tracer uptake and kinetics. 


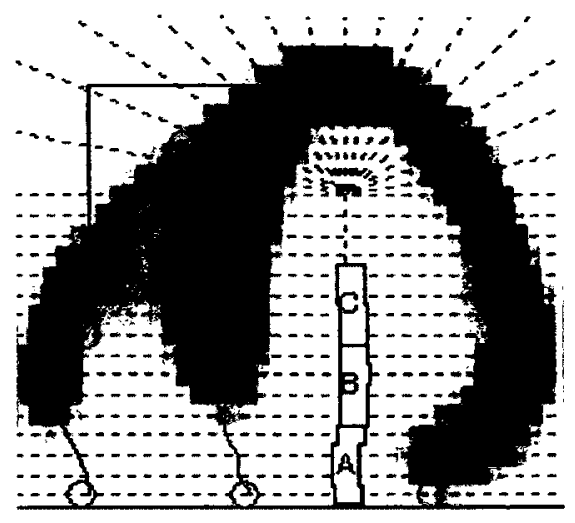

Figure 5-2 : HLA slice indicating a case interference of the fitting algorithm by spillover from the RV.

5) Dependence of LV reorientation and registration: In cases where the reorientation of the LV was not correctly defined, automatic registration yielded poor results. Automatic LV reorientation appears to be adversely impaired in $\mathrm{PH}$ cases requiring operator intervention. Reorientation was beyond the scope of this work and may warrant future investigation. Because of the direct dependence of estimating initial location of control points on LV registration, the algorithm was also directly affected when there is a poor LV fit.

In spite of these challenges, the results indicate that in about $90 \%$ of the cases the model fit was appropriate in the free wall region, particularly around the midsection, despite the wide range of morphologies. In light of major current interest being focused on molecular function quantification in the midsection free wall, the performance of the automated fitting algorithm is somewhat encouraging. In other RV regions significant user interaction is required resulting in increased operator variability. However, the integrated GUI with few control points enables the operator to quickly manipulate the RV model to accommodate almost any RV shape and size resulting in a timely and useful image analysis tool. 


\subsection{Operator Variability}

Operator variability is an important component of measurement variability and its characterization is essential for determining detectable population differences and population sizes in future experiments. Operator variability results indicated a good agreement between repeated measurements for each operator as well as between the two operators. The rest of this chapter will discuss in-depth results of operator variability.

\subsubsection{User Intervention}

Frequent operator adjustment at the apical region of the RV suggests that diverse range of RV morphologies imposes a challenge to the fitting algorithm. A wide range of $\mathrm{RV}$ extent control point are required to accommodate the range of physiologies as demonstrated in Figure 5-3 for three different patient images. While the extent control point was restricted to longitudinal motion during automatic registration, during operator intervention it could be moved both laterally and longitudinally. While two dimensional controls can effectively accommodate a wider range of RV shape, it potentially results in non-unique placement, overwhelming the user, and increasing sampling point location variability in the apical region.
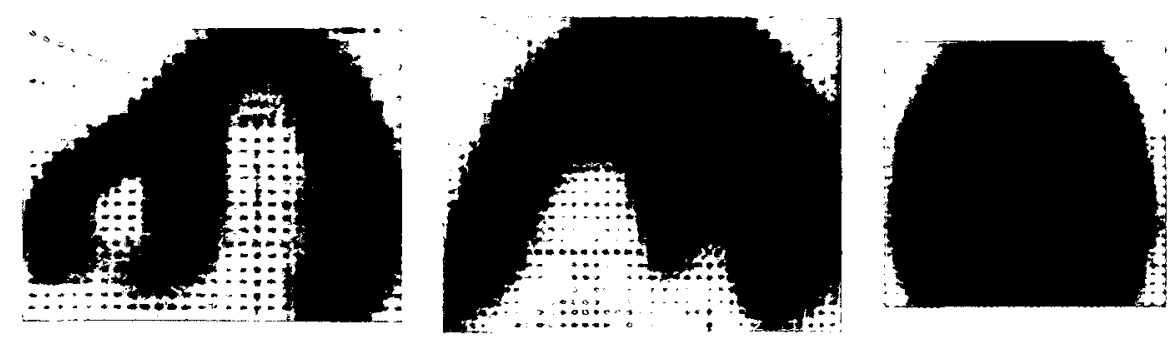

Figure 5-3 : HLA slices demonstrating a variation of the location of extent control point. The Wide range of RV anatomies that the registration utility has to accommodate diminishes the possibility of constraining for optimization algorithm. 
Frequent operator intervention in the atrial region by both operators can be partially attributed to low intensity and indistinctive RV boundary around the atrial region. Furthermore, the presence of the base of the pulmonary trunk in proximity of the right atrium and on the same short axis planes also complicates clear delineation of the $\mathrm{RV}$ boundaries towards the atrium, resulting in arbitrarily placement of the spline point as demonstrated in Figure 5-4. Low image contrast and complicated structure in the atrial region make sampling to the atria unreliable with current imaging technologies. Improvements in imaging technologies, more specific tracers, and a more robust right atrial model may contribute to more accurate sampling of the atrium.

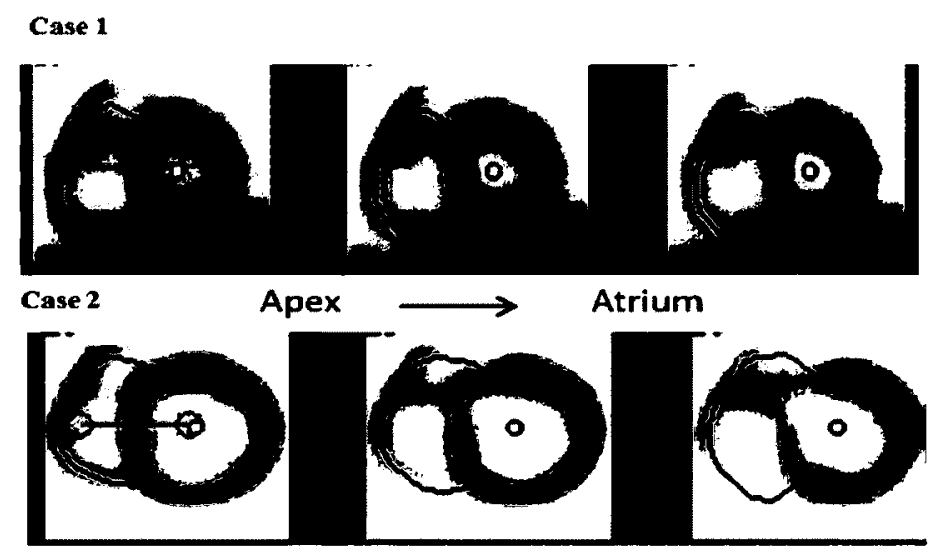

Figure 5-4 : SA slices shows 2 cases of indistinguishable shape of the RV towards the atrium due to the base of the pulmonary trunk.

It is possible that the number of interventions were influenced by the level of understanding of the application. Operator 2 was a novice user of the software, and intervened more frequently than Operator 1 . This may suggest that in cases where the boundary is ambiguous, particularly at the apex or atrium regions, the novice may have been indecisive, resulting in more intervention, and less reproducible results. Both operators intervened more in the second processing session, suggesting that the user intervention increased as their comfort level with the software increased. 


\subsubsection{Intra-Operator Variability}

The distal region had higher variability for both operators. While this suggests a high level of intervention in the territory, it also indicates some level independence between the two test performed by each operator. As highlighted before the apical region of the RV exhibit more anatomical variations particularly in hypertrophic cases and influences the general shape of the RV. Because of the fixed number of RV ROI slices, longitudinal shifting of the extent control point alters the slice thickness and position of all sampling points. Other control points have a more local effect. The automatic registration algorithm was noted to perform more poorly in this region, subsequently requiring more user intervention. While user intervention does not justify the magnitude of error, its frequency increases the number of errors and therefore the average magnitude.

For both operators, $95 \%$ of sample point positions varied by less than $5 \mathrm{~mm}(\sim 1.5$ pixels) between runs in all territories, which is not a drastic effect considering that the reconstructed image resolution for ${ }^{82} \mathrm{Rb}$ in $\sim 12 \mathrm{~mm}$ FWHM (full width at half maximum) and $>8 \mathrm{~mm}$ with good quality ${ }^{18} \mathrm{~F}$ labeled tracers. For operator 1 (expert), the hemi polar map indicates less variability compared to operator 2 (novice). This does not suggest better results from either user but rather explains the variation introduced by different behaviors between the two operators in using the application, indicating that operator training may be beneficial to reducing operator variability.

For both operators the medial region indicated low variability among all vascular territories. This is consistent with the fact that the medial region corresponding to the free wall is usually well defined and relatively distal from other interfering structures in the 
image. As indicated by validation of automatic fitting results, the spline model performs better in this region. This decreases the frequency of user intervention as reported in Table 4-9, which also contributed to improved operator dependent reproducibility in this region. From the linear regression plots for both users (Figure 4-4), correlation in this region was good $\left(r^{2}>0.94\right)$

The reproducibility coefficient of relative tracer uptake for inter operator agreement for operator 1 (novice) was $5.6 \%$ slightly less than that of operator 2 (novice) which was $6.4 \%$. Again, this indicates higher variability in the novice operator than in the expert operator and with more training the operator reproducibility might be improved $[46,48]$.

\subsubsection{Inter-Operator Variability}

Similar regional patterns were observed between inter- and intra-operator variability with larger operator variability in the distal (apical) region. From relative uptake standard deviation hemi polar map, it is obvious that the least placement variability occurred in the medial territory which is consistent with the observation that algorithm performs better in the free wall region. While the improved reproducibility in the free wall can be attributed to good performance of the automatic fitting algorithm, it is also possible that, since the free wall anatomy is less ambiguous and less susceptible to spillover effects from the LV therefore operators are likely to produce congruent results when adjusting control points in this region. As in intra-operator variability, $85 \%$ sample points' positions varied by less than $5 \mathrm{~mm}$.

The relative uptake reproducibility coefficient (RPC) was found to be $8.2 \%$, which is not significantly different from intra-operator values $(\mathrm{p}=\mathrm{ns})$ as shown in Table 
5-1. Inter-operator reproducibility may be improved through training, and standardization.

\subsection{Validation of Cavity Volumes}

Comparison between cavity volumes and EF measured from PET images with FlowQuant $^{\mathfrak{Q}}$ and CMR images with Argus suggests excellent agreement. There is still some variability in measurements primarily due to the fact that image processing is manual, processed by different operators with images acquired by different imaging modalities.

Slight differences in volume measurements can also be due to estimation of the location of the valve plane as well poor estimation of the endocardial walls [56]. For this research the wall thickness of the RV was assumed to be half of the LV wall thickness. This assumption may be reasonable in normal hearts but may underestimate RV wall thickness in hypertrophic hearts. Furthermore, in this work the valve plane was modeled as a flat short axis plane at a roughly estimated $z$-slice based on the reorientation stage. In most images, intensity diminishes rapidly towards the atrium (Figure 5-5) which in future work may be used to position the plane in an oblique angled plane that is determined by image information. More accurate RV valve plane modeling may yield more accurate RV cavity volumes, which in future work should be evaluated using a gold standard modality such as CMR, CT, or echocardiography.

An important limitation of this work is the small data size. Only five confirmed PH patients were identified in our study and had undergone both PET and CMR scans. These small numbers are in part due to the prevalence of $\mathrm{PH}$, the ability for early 
detection, and the high associated mortality and morbidity which adversely affect patient enrollment and maintenance in the study. These complications highlight the need for multi-center studies in order to obtain large datasets.

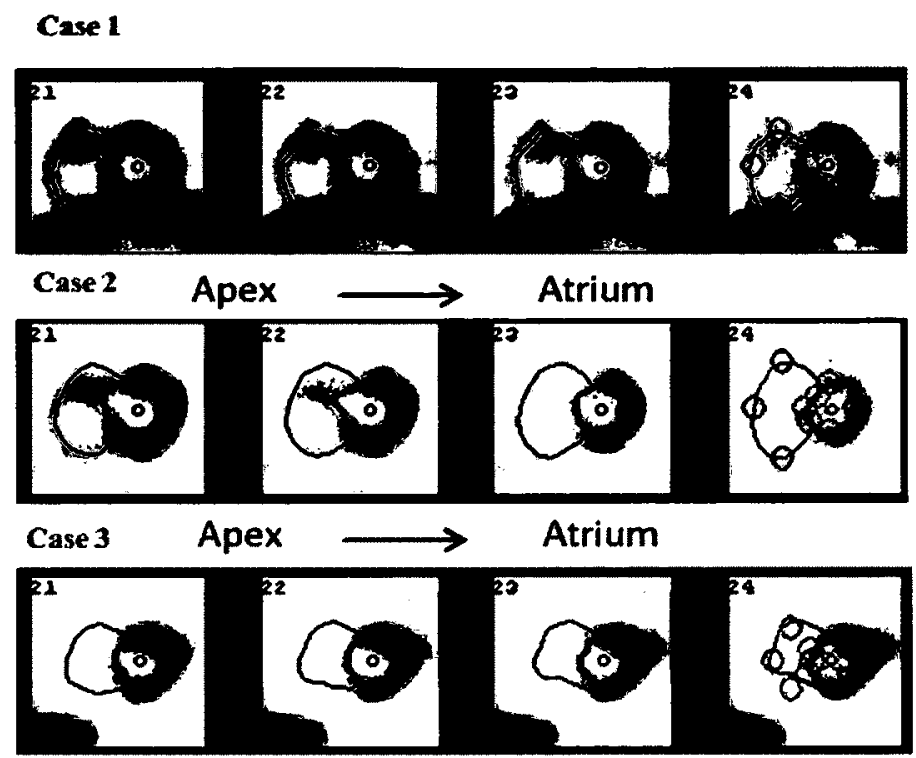

Figure 5-5 : SA slices shows 3 cases indicating diminishing image intensity of RV ROI toward the atrium.

Table 5-2 compares RV cavity volumes and EF measured in our PH population to previously reported $\mathrm{PH}$ and normal populations. The EF in our $\mathrm{PH}$ population is comparable to the previously reported $\mathrm{EF}$ for $\mathrm{PH}$ in and much smaller than normal RV EF. Cavity volumes in our study were significantly lower than those reported by Grapsa et al for PH patients $(p=0.290)$, and not significantly different from the normal population $(p=n$.s. $)$ [47]. Our study population was specifically selected for early disease state using pulmonary arterial pressure $>14 \mathrm{mmHg}$ as a criterion compared to $>30 \mathrm{mmHg}$ [47]. It is therefore likely that the PH population reported by Grapsa et al had more advanced and severe disease consistent with much enlarged RV volumes. 
Table 5-2 : Comparison of Cavity volumes and EF between current research and results from research by Grapsa et al for PH patients [22].

\begin{tabular}{|l|l|l|l|}
\hline Measurement & Current Research & Grapsa et al (CMR) \\
\hline & FlowQuant $(\mathrm{n}=5)$ & $\mathrm{PH}(\mathrm{n}=20)$ & Normal $(\mathrm{n}=60)$ \\
\hline R-EDV $(\mathrm{mL})$ & $90 \pm 25(83-119)$ & $187 \pm 41(99-279)$ & $94 \pm 15(64-112)$ \\
\hline R-ESV $(\mathrm{mL})$ & $61 \pm 78(23-90)$ & $126 \pm 36(55-223)$ & $54 \pm 11(37-75)$ \\
\hline R-EF (\%) & $37 \pm 13(28-57)$ & $31 \pm 9(18-54)$ & $62 \pm 13(37-85)$ \\
\hline
\end{tabular}

\subsection{Dynamic Analysis}

While not the primary focus of this work, application of RV ROIs to dynamic images can facilitate extraction of regional RV tissue time-activity curves (TACs), as is the cutting edge in LV physiologic function quantification. RV TACs can be processed using dynamic analysis techniques, such as kinetic-modeling to quantify physiologic functions such as myocardial blood flow, metabolism rates, and receptor densities. While the topic of kinetic analysis is beyond the scope of this work, an example is included for demonstration in Figure 5-6 to emphasize the utility of this work.

Arterial blood time-activity curves (TACs) are obtained by a region of interest (ROI) in the center of the LV cavity shown in Figure 5-7 as white rectangles labeled A, $\mathrm{B}$, and $\mathrm{C}$. The blood input function represents an input function to the kinetic model. ROIs in the RV and LV myocardium are also applied to the dynamic image to sample TACs for each polar map (PM) segment. The myocardial TACs represent the output function of the kinetic model. Representative blood and myocardium TACs are shown in the lower part of Figure 5-6 in red and blue respectively. 

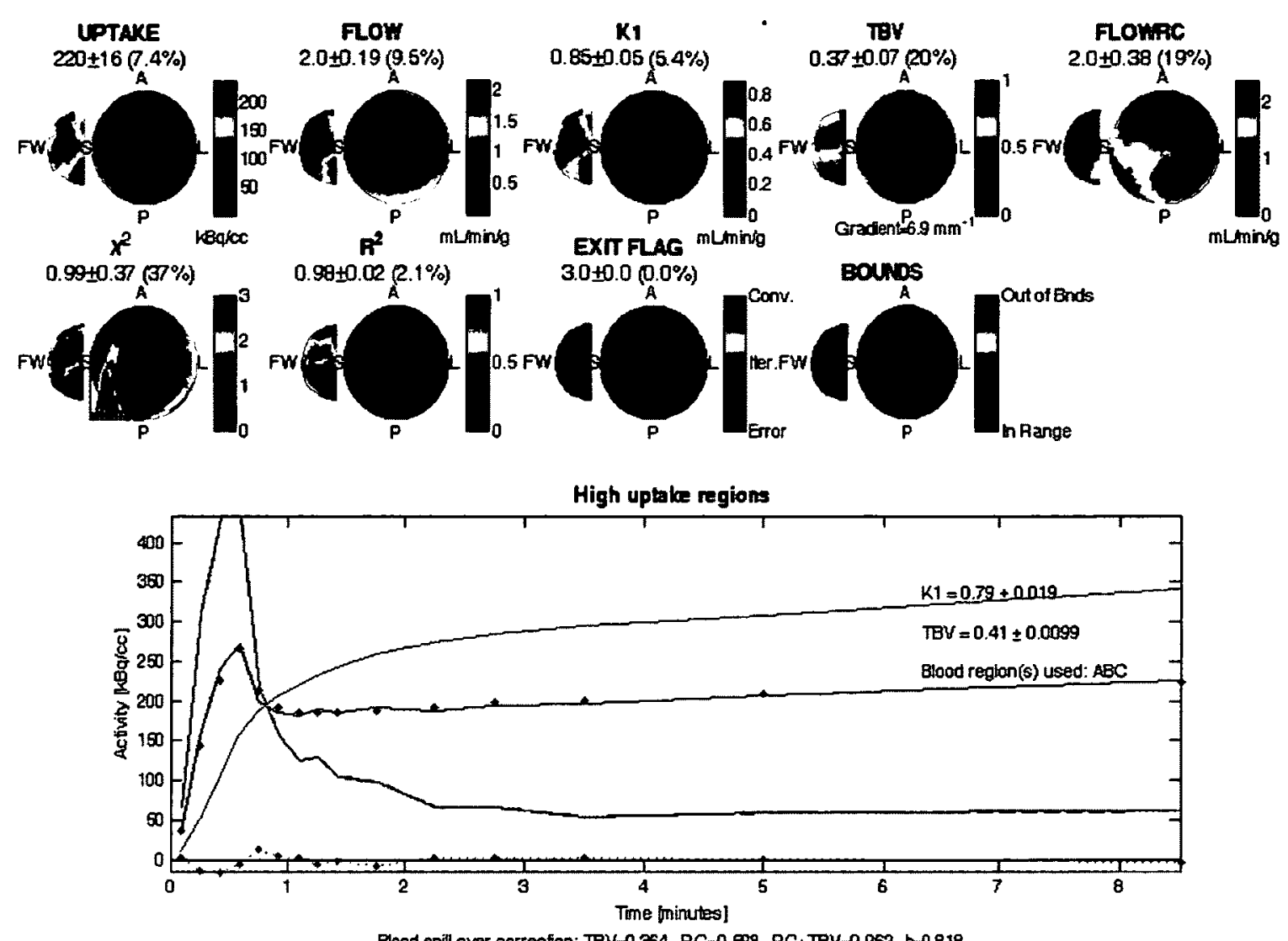

Blood spill over correction: $T B V=0.364 \quad R C=0.598 \quad R C+T B V=0.962 \quad b=0.818$

Figure 5-6 : A sample Kinetic modeling integrated report for the LV and RV. Uptake polar maps for both $\mathrm{RV}$ and $\mathrm{LV}$ are shown at the top half of the figure and time activity curves are shown at the bottom of the curve.

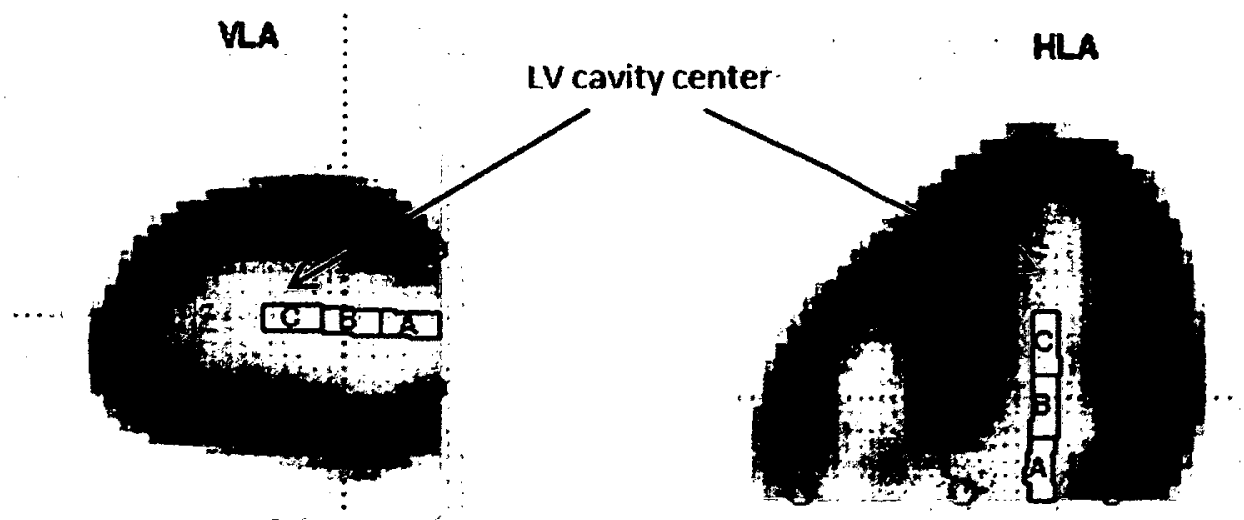

Figure 5-7 : Vertical Long Axis (VLA) view of LV and Horizontal Long Axis (HLA) view of both the LV and RV. The center of $L V$ cavity is depicted by labeled $A, B$, and $C$. 
In each PM segment kinetic-model parameters are fit to the global input TAC and segment specific output TAC to generate a local estimate of the kinetic parameters. In the case of Figure 5-6 K1 and TBV are the kinetic parameters solved using the demonstrated

${ }^{82} \mathrm{Rb}$ 1-compartment fixed distribution volume (DV) model, and are shown as respective PMs in the top row. MBF values are estimated by correcting $\mathrm{K} 1$ values using a precalibrated extraction correction function, and indicate the amount of blood supplied to the tissue $[\mathrm{mL} / \mathrm{min} / \mathrm{g}]$. Other goodness of fit parameters (i.e. $X^{2}, \mathrm{R}^{2}$, optimization exit flag, and boundary conditions), are also reported for each segment for quality assurance (lower row of PMs in Figure 5-6). MBF (shown as FLOW PM) can be used to detect disease that may otherwise by missed using conventional uptake imaging (uptake PM also shown in top row).

This example demonstrates a naïve attempt at quantifying MBF in the RV territory. Additional work is required to validate and characterize MBF quantification in the RV. This author speculates that the kinetic model may need to be modified to account for spill-in signal from the RV blood cavity (proximal to the RV myocardium), which is not accounted for in the existing model. The inadequacy of the existing model is highlighted by out of bound parameters and low $R^{2}$ values in parts of the RV hemi-polar map. This aspect is not addressed as part of this work.

\subsection{Image Quality}

Previous work on RV analysis focused primarily on SPECT imaging while this research focused on PET imaging technology and is expected to be easily applicable to 
SPECT imaging. Both modalities generate blurry images with a signal to noise ration that is approximately 10. Image blur results in spatial resolution that on the same order as LV myocardial feature sizes (e.g. $\sim 12 \mathrm{~mm}$ and $\sim 1.2 \mathrm{~mm}$ for FDG PET in humans and rats respectively)[63] which limits the ability to resolve small RV feature sizes, especially in normal subjects. The performance of these technologies is expected to improve with technological advances, PET is likely to continue to exhibit superior sensitivity and temporal resolution to SPECT, and while both modalities will provide improved spatial resolution, small animal SPECT is likely to exhibit superior spatial resolution to PET State of the art small animal PET and SPECT cameras have recently been reported to achieve $0.5 \mathrm{~mm}$ and $0.2 \mathrm{~mm}$ spatial resolution respectively $[62,64,65]$. These technological improvements are expected to directly influence the performance of this project in a positive manner.

\subsection{Future work}

The validation of the results also revealed that there is still some significant level of intervention required during the analysis process. Though it may not be possible to completely eliminate user intervention, the automatic optimization algorithm can likely be improved. This will require more research of the relation between RV and LV sizes and shape particularly in hypertrophic cases to help provide better initial estimates of location of control points. If the analysis software can correctly infer the heart condition of the image under analysis, extreme hypertrophic, mild hypertrophic or normal, it can produce a reasonable initial estimate. Good initial estimates of location of control points 
reduce the convergence time of the automatic search and optimization algorithm. It also minimizes the number and complexity of constraints imposed on the algorithm.

Furthermore, additional model constraints should be explored to help the optimization algorithm converge, with improved robustness to local minima of the cost function. As part of the enhancing performance of the algorithm, it may be possible to focus the registration only on territories which care clinically significant. For example, if the atrial region does not have significant influence on functional analysis of the RV, registration of the RV should be done up to the midsection while ignoring the atrium.

Gated analysis currently involves much operator intervention; improving automation will provide more accurate and reproducible results as well as reduce operator variable. Automation of the software will also reduce the analysis time..

As demonstrated in the methods section preliminary work has been started to apply the RV region of interest in quantification of the RV function.

\subsection{Impact of Work}

To the best of our knowledge, no clinical or research software currently provides fully automatic definition of RV ROI. Current clinically validated cardiac analysis tools including Argus (Siemens, Erlangen, Germany) require tedious (20-40 minutes per gated CMR image) manual delineation of the RV. Even FlowQuant (UOHI, Ottawa, Canada) which is highly automated for LV ROI definition requires some level of operator intervention $(\sim 10 \%$ of images). Thus, the degree of operator intervention that was demonstrated in this work leans favorably towards a high degree of automation and low operator work load ( $<5 \mathrm{~min}$ per gated scan and $<2$ min per static and dynamic scans). 
Earlier research work on RV function including a study by Aepfelbacher et al assessed RV function and structure using SPECT in comparison with echocardiography and produced conclusive results for diseased RV [1]. On the contrary, despite having only 5 PH subjects to work with, our results indicates a good correlation $\left(r^{2}>0.95\right)$ between PET and CMR for cavity volumes an indication that this utility can be used to reliable evaluate RV cardiac function. 


\section{Chapter: Conclusions}

The RV is a critical component of the pulmonary circulatory system, and understanding its molecular function especially in $\mathrm{PH}$ patients may be useful to understanding disease, accurate early diagnosing of patients, prognosticating, and directing therapy. The use of nuclear imaging technology for functional analysis of cardiac function of the LV is well founded and with recent improvements in imaging technologies may be applied to the right ventricle. However, the anomalous shape of the RV and wide range of morphologies creates a major challenge in attempting to adapt existing LV analysis functions to RV analysis. This research successfully established a model that can be used to segment the RV region of interest in cardiac PET images, a critical step to functional analysis of the RV.

A spline model was developed, validated, and characterized, and was demonstrated to sufficiently registers the RV region of interest semi-automatically.

We have demonstrated that this utility can be used for gated image analysis to deduce the RV ROI volume which is used to derive RV cavity volumes and EF comparable to the results produced using CMR. While excellent agreement of cavity volume and ejection fraction measurements $\left(r^{2}=0.9535\right.$ and 0.965 respectively, $\left.n=5\right)$, more validation is required.

Operator dependent reproducibility was high and comparable with existing LV analysis software. The end result is a useful tool, fully integrated into an existing, established software package. This tool is useful for research in RV dysfunction and 
disease and also serves as a development platform for future RV image analysis tools including physiologic function quantification using dynamic imaging.

To the best of our knowledge, this tool is unique in its ability to define the RV ROI in cardiac images. Tools of this type are important to understanding RV disease mechanisms and progression, and to evaluating therapies from cardiac PET images. Overall, we are confident that this tool may be used in current research and in the future it may be instrumental to diagnose disease in clinic and to guide therapy for improved prognosis and quality of life of patients suffering of RV related ailments. 


\section{Chapter: Bibliography or References}

[1] F. C. Aepfelbacher, S. B. Yeon, K. K. L. Ho, J. A. Parker, and P. G. Danias, "ECGGated 99mTc Single-Photon Emission CT for Assessment of Right Ventricular Structure and Function?", Chest, vol. 124, no. 1, pp. $227-232$, Jul. 2003.

[2] A. Gomez, D. Bialostozky, A. Zajarias, E. Santos, A. Palomar, M. L. Martinez, and J. Sandoval, "Right ventricular ischemia in patients with primary pulmonary hypertension", Journal of the American College of Cardiology, vol. 38, no. 4, p. 1137, 2001.

[3] G. S. Lin, H. H. Hines, G. Grant, K. Taylor, and C. Ryals, "Automated Quantification of Myocardial Ischemia and Wall Motion Defects by Use of Cardiac SPECT Polar Mapping and 4-Dimensional Surface Rendering", Journal of Nuclear Medicine Technology, vol. 34, no. 1, pp. 3-17, Mar. 2006.

[4] F. Mannting, Y. V. Zabrodina, and C. Dass, "Significance of Increased Right Ventricular Uptake on $99 \mathrm{mTc}$-Sestamibi SPECT in Patients with Coronary Artery Disease", Journal of Nuclear Medicine, vol. 40, no. 6, pp. 889-894, Jun. 1999.

[5] N. Pereira, W. S. Klutz, R. E. Fox, K. W. Ahn, and M. I. Travin, "Identification of severe right ventricular dysfunction by technetium-99m-sestamibi gated SPECT imaging", Journal of Nuclear Medicine, vol. 38, no. 2, p. 254, 1997.

[6] R. Klein, M. Lortie, A. Adler, R.S. Beanlands, R. deKemp, "Fully Automated Software for Polar-Map Registration and Sampling from PET Images", Nuclear Science Symposium Conference Record, 2006.

[7] K. Saha, J.A. Case, S.J. Cullom, T. Bateman, Bai-Ling Hsu, “Automated Detection of Myocardium Boundary in Rb-82 PET Images Using Wavelet Based Approach", 
Nuclear Science Symposium Conference Record, 2006. IEEE, vol.4, pp.2068-2071, 2006.

[8] A. Khorsand, S. Graf, C. Pirich, G. Wagner, D. Moertl, H. Frank, K. Kletter, H. Sochor, G. Maurer, E. Schuster, G. Porenta, "Image analysis of gated cardiac PET to assess left ventricular volumes and contractile function", Computers in Cardiology 2000 , pp.311-314, 2000.

[9] P. Kaufmann, "Measurement of left ventricular volumes and function using O-15-labeled carbon monoxide gated PET", Journal of Nuclear Cardiology, vol. 12, Nov. 2005, pp. 620-621. [10] J. Machac, "PET myocardial perfusion imaging", Am J Card Imaging, vol. 6, 1992, pp. 42-58.

[11] N. Preumont, J. Jansens, G. Berkenboom, P. van de Borne, E. Stoupel, and S. Goldman, "Effects of right ventricular pacing on regional myocardial glucose metabolism", Europace, vol. 7, Nov. 2005, pp. $584-591$.

[12] M.P. Feneley, J.R. Elbeery, J.S. Rankin, "An ellipsoidal shell subtraction model of the right ventricle: validation and application in vivo", Engineering in Medicine and Biology Society, 1989. Images of the Twenty-First Century., Proceedings of the Annual International Conference of the IEEE, vol.1, pp.124-125 9-12 Nov 1989.

[13] N.C. Chesler, A. Roldan, R.R. Vanderpool, R. Naeije, "How to measure pulmonary vascular and right ventricular function", Engineering in Medicine and Biology Society, 2009. EMBC 2009. Annual International Conference of the IEEE, vol., no., pp.177-180, 3-6 Sept. 2009.

[14] D. Craig, R. Uppal, D. Glower, C. Straley, T. Hampton, A. Pasipoularides, P.K Smith, "Simultaneous computation of right and left ventricular systolic function", Computers in 
Cardiology 1992. Proceedings. , vol., no., pp.511-514, 11-14 Oct 1992

[ [15] R. deKemp, R. Klein, J. Renaud, A. Alghamdi, M. Lortie, J.N DaSilva, R. Beanlands, “3D list-mode cardiac PET for simultaneous quantification of myocardial blood flow and ventricular function", Nuclear Science Symposium Conference Record, 2008. NSS '08. IEEE, vol., no., pp.5215-5218, 19-25 Oct. 2008.

[16] M. Margadan-Mendez, A. Juslin, S.V. Nesterov, K. Kalliokoski, J. Knuuti, U. Ruotsalainen, "ICA Based Automatic Segmentation of Dynamic Cardiac PET Images", Information Technology in Biomedicine, IEEE Transactions on , vol.14, no.3, pp.795-802, May 2010.

[17] M. A .Lodge, H. Braess, F. Mahmoud, J. Suh, N. Englar, S. Geyser-Stoops, J. Jenkins, S. L. Bacharach, V. Dilsizian, "Developments in nuclear cardiology: transition from single photon emission computed tomography to positron emission tomography-computed tomography", J Invasive Cardiology vol.17, no. 9, pp. 491-496 September 2005.

[18] A. K. Paul, H. A. Nabi "Gated Myocardial Perfusion SPECT: Basic Principles, Technical Aspects, and Clinical Applications" J. Nucl. Med. Technol. 2004 32:179-187

[19] F. Haddad, S. A. Hunt, D. N. Rosenthal, and D. J. Murphy, "Right Ventricular Function in Cardiovascular Disease, Part I", Circulation, vol. 117, no. 11, pp. 1436-1448, Mar. 2008.

[20] B. J. W. Chow, K. Ananthasubramaniam, R. A. deKemp, M. M Dalipaj, R. S. B. Beanlands, T.D Ruddy, "Comparison of treadmill exercise versus dipyridamole stress with myocardial perfusion imaging using rubidium-82 positron emission tomography”, J Am Coll Cardiol vol.45,pp.1227-1234, 2005

[21 ] F.L. Arend, Schinkel, D. Poldermans, A. Elhendy, and J. J. Bax,“Assessment of Myocardial Viability in Patients with Heart Failure”, J Nucl Med vol.48, pp.1135-1146,July 
2007.

[22] J. Grapsa, D. P. O'Regan, H. Pavlopoulos, G. Durighel, D. Dawson, and P. Nihoyannopoulos, "Right ventricular remodelling in pulmonary arterial hypertension with three-dimensional echocardiography: comparison with cardiac magnetic resonance imaging", European Journal of Echocardiography, vol. 11, no. 1, pp. 64-73, 2010.

[23] R. Klein, J. M. Renaud, M. C. Ziadi, S. L. Thorn, A. Adler, R. S., Beanlands, R. A., deKemp, "Intra- and inter-operator repeatability of myocardial blood flow and myocardial flow reserve measurements using Rubdium-82 PET and a highly automated analysis program.", J. Nucl. Cardiol., 2010;17(4):600-616.

[24] N.C. Chesler, A. Roldan, R.R. Vanderpool, R. Naeije, "How to measure pulmonary vascular and right ventricular function", Engineering in Medicine and Biology Society, 2009. EMBC 2009. Annual International Conference of the IEEE , vol., no., pp.177-180, 3-6 Sept. 2009

[25] D. Metaxas, L. Axel, A. Montillo, K. Park, "Automated segmentation and motion estimation of LV/RV motion from MRI", Engineering in Medicine and Biology, 2002. 24th Annual Conference and the Annual Fall Meeting of the Biomedical Engineering Society EMBS/BMES Conference, 2002. Proceedings of the Second Joint, vol.2, no., pp. 1099- 1100 vol.2, 2002

[26] L. Sugeng, H.J. Nesser, L. Weinert, J. Niel, C. Ebner, R. Steringer-Mascherbauer, R. Bartolles, R. Baumann, G. Schummers, R.M. Lang, V. Mor-Avi, "Multimodality comparison of quantitative volumetric analysis of the right ventricle", Computers in Cardiology, 2009, vol., no., pp.29-32, 13-16 Sept. 2009

[27] J. Schaerer, Y. Rouchdy, P. Clarysse, B. Hiba, P. Croisille, J. Pousin, I. E. Magnin, 
"Simultaneous segmentation of the left and right heart ventricles in 3D cine MR images of small animals", Computers in Cardiology, 2005, vol., no., pp.231-234, 25-28 Sept. 2005

[28] L. Xia, M. Huo, F. Liu, B. He, S. Crozier, "Motion analysis of right ventricular wall based on an electromechanical biventricular model", Engineering in Medicine and Biology Society, 2004. IEMBS '04. 26th Annual International Conference of the IEEE, vol.1, no., pp.898-901, 1-5 Sept. 2004

[29] Z.A. Fayad, V.A. Ferrari, D.L. Kraitchman, A.A. Young, D.C. Bloomgarden, L. Axel, "Right ventricular wall mechanics in normals and patients with pulmonary hypertension using magnetic resonance tissue tagging", Computers in Cardiology 1995 , vol., no., pp.233-236, $10-$ 13 Sept. 1995

[30] F. Haddad, S. A. Hunt, D. N. Rosenthal, and D. J. Murphy, "Right Ventricular Function in Cardiovascular Disease, Part I", Circulation, vol. 117, no. 11, pp. 1436 -1448, Mar. 2008.

[31] C. Treacy, E. Soon, G. Hagan, J. Coulson, J. Rudd, J. Pepke-Zaba, K. Sheares, M. Southwood, N. Screaton, N. Morrell, and R. Ross, "18FDG PET imaging can quantify increased cellular metabolism in pulmonary arterial hypertension: A proof-of-principle study", Pulmonary Circulation, no. 4, p. 448.

[32] M. Oikawa, Y. Kagaya, H. Otani, M. Sakuma, J. Demachi, J. Suzuki, T. Takahashi, J. Nawata, T. Ido, J. Watanabe, and others, "Increased [18F] fluorodeoxyglucose accumulation in right ventricular free wall in patients with pulmonary hypertension and the effect of epoprostenol", Journal of the American College of Cardiology, vol. 45, no. 11, p. 1849, 2005.

[33] R. Kluge, H. Barthel, H. Pankau, A. Seese, J. Schauer, H. Wirtz, H.-J. Seyfarth, J. Steinbach, O. Sabri, and J. Winkler, "Different Mechanisms for Changes in Glucose Uptake of the Right and Left Ventricular Myocardium in Pulmonary Hypertension", Journal of Nuclear 
Medicine, vol. 46, no. 1, pp. 25 -31, Jan. 2005.

[34] R. Wensel, C. F. Opitz, S. D. Anker, J. Winkler, G. Höffken, F. X. Kleber, R. Sharma, M. Hummel, R. Hetzer, and R. Ewert, "Assessment of Survival in Patients With Primary Pulmonary Hypertension", Circulation, vol. 106, no. 3, pp. 319 -324, Jul. 2002.

[35] A. Vonk-Noordegraaf, J. W. Lankhaar, M. J. W. Götte, J. T. Marcus, P. E. Postmus, and N. Westerhof, "Magnetic resonance and nuclear imaging of the right ventricle in pulmonary arterial hypertension", European Heart Journal Supplements, vol. 9, no. suppl H, pp. H29-H34, Dec. 2007.

[36] J. W. Lankhaar, N. Westerhof, T. J. C. Faes, K. M. J. Marques, J. T. Marcus, P. E. Postmus, and A. Vonk-Noordegraaf, "Quantification of right ventricular afterload in patients with and without pulmonary hypertension", American Journal of Physiology - Heart and Circulatory Physiology, vol. 291, no. 4, pp. H1731 -H1737, Oct. 2006.

[37] R. Benza, R. Biederman, S. Murali, and H. Gupta, "Role of Cardiac Magnetic Resonance Imaging in the Management of Patients With Pulmonary Arterial Hypertension", Journal of the American College of Cardiology, vol. 52, no. 21, pp. 1683-1692, Nov. 2008.

[38] L. J. Dell'Italia and W. P. Santamore, "Can indices of left ventricular function be applied to the right ventricle?", Progress in Cardiovascular Diseases, vol. 40, no. 4, pp. 309-324, Jan. 1998.

[40] S. A. van Wolferen, J. T. Marcus, A. Boonstra, K. M. J. Marques, J. G. F. Bronzwaer, M. D. Spreeuwenberg, P. E. Postmus, and A. Vonk-Noordegraaf, "Prognostic value of right ventricular mass, volume, and function in idiopathic pulmonary arterial hypertension", European Heart Journal, vol. 28, no. 10, pp. 1250 -1257, May 2007.

[41] G. R. Stevens, A. Garcia-Alvarez, S. Sahni, M. J. Garcia, V. Fuster, and J. Sanz, "RV 
Dysfunction In Pulmonary Hypertension Is Independently Related To Pulmonary Artery Stiffness", JACC: Cardiovascular Imaging, vol. 5, no. 4, pp. 378-387, Apr. 2012.

[42] C. Hough, C. Kurtz, D. Ralph, F. Sheehan, M.-P. Waiss, and P. Leary, "Three-dimensional analysis of right ventricular shape and function in pulmonary hypertension", Pulmonary Circulation, vol. 2, no. 1, pp. 34-40, Jan. 2012.

[43] R. O. Bonow, G. Maurer, K. L. Lee, T. A. Holly, P. F. Binkley, P. Desvigne-Nickens, J. Drozdz, P. S. Farsky, A. M. Feldman, T. Doenst, R. E. Michler, D. S. Berman, J. C. Nicolau, P. A. Pellikka, K. Wrobel, N. Alotti, F. M. Asch, L. E. Favaloro, L. She, E. J. Velazquez, R. H. Jones, and J. A. Panza, "Myocardial Viability and Survival in Ischemic Left Ventricular Dysfunction”, N Engl J Med, vol. 364, no. 17, pp. 1617-1625, Apr. 2011.

[44 ] S. G. Nekolla, T. Balbach, J. Neverve, F. G. Bengel, M. Schwaiger, "Quantitative cardiac PET imaging: reproducibility in an integrated analysis environment", Nuclear Science Symposium Conference Record, 2000 IEEE , vol.3, no., pp.18/77-18/80 vol.3, 2000.

[45] S. Mantha, M. F. Roizen, L. A. Fleisher, R. Thisted, and J. Foss, "Comparing Methods of Clinical Measurement: Reporting Standards for Bland and Altman Analysis", Anesthesia \& Analgesia, vol. 90, no. 3, pp. $593-602$, Mar. 2000.

[46] S. Halligan, "Reproducibility, repeatability, correlation and measurement error", British Journal of Radiology, vol. 75, no. 890, pp. $193-194$, Feb. 2002.

[47] J. C. Paeng, D. S. Lee, G. J. Cheon, M. M. Lee, J. K. Chung, and M. C. Lee, "Reproducibility of an Automatic Quantitation of Regional Myocardial Wall Motion and Systolic Thickening on Gated 99mTc-Sestamibi Myocardial SPECT", Journal of Nuclear Medicine, vol. 42, no. 5, pp. $695-700$, May 2001.

[48] A. Furlan, A. Patil, B. Park, C. C. H. Chang, M. S. Roberts, and K. T. Bae, “Accuracy and 
Reproducibility of Blood Clot Burden Quantification With Pulmonary CT Angiography", American Journal of Roentgenology, vol. 196, no. 3, pp. 516 -523, Mar. 2011.

[50] J. G. Gomez-Arroyo, L. Farkas, A. A. Alhussaini, D. Farkas, D. Kraskauskas, N. F. Voelkel, and H. J. Bogaard, "The monocrotaline model of pulmonary hypertension in perspective", American Journal of Physiology - Lung Cellular and Molecular Physiology, vol. 302, no. 4, pp. L363-L369, Feb. 2012.

[51] R. J. Hicks, V. Kalff, V. Savas, M. R. Starling, and M. Schwaiger, "Assessment of right ventricular oxidative metabolism by positron emission tomography with $\mathrm{C}-11$ acetate in aortic valve disease", The American Journal of Cardiology, vol. 67, no. 8, pp. 753-757, Apr. 1991.

[52] J. Chiba, Y. Takeishi, S. Abe, H. Tomoike, "Visualisation of exercise-induced ischaemia of the right ventricle by thallium-201 single photon emission computed tomography", Heart. 1997 January; 77(1): 40-45.

[54] A. Lakoma, D. Tuite, J. Sheehan, P. Weale, and J. C. Carr, "Measurement of Pulmonary Circulation Parameters Using Time-Resolved MR Angiography in Patients After Ross Procedure", American Journal of Roentgenology, vol. 194, no. 4, pp. 912-919, Apr. 2010.

[55] C. Plumhans, G. Mühlenbruch, A. Rapaee, K. H. Sim, T. Seyfarth, R. W. Günther, and A. H. Mahnken, "Assessment of Global Right Ventricular Function on 64-MDCT Compared with MRI", American Journal of Roentgenology, vol. 190, no. 5, pp. 1358-1361, May 2008.

[56] G. Domenighetti, V. Saglini, "Short- and long-term hemodynamic effects of oral nifedipine in patients with pulmonary hypertension secondary to COPD and lung fibrosis, deleterious effects in patients with restrictive disease", CHEST 1992;102(3):708-714.

[57] K. Rajappan, L. Livieratos, P. G. Camici, and D. J. Pennell, "Measurement of Ventricular Volumes and Function: A Comparison of Gated PET and Cardiovascular Magnetic 
Resonance", Journal of Nuclear Medicine, vol. 43, no. 6, pp. 806-810, Jun. 2002.

[58] R. Naeije and S. Huez, "Right ventricular function in pulmonary hypertension: physiological concepts", European Heart Journal Supplements, vol. 9, no. suppl H, p. H5, 2007. [59] M. D. Cerqueira, N. J. Weissman, V. Dilsizian, A. K. Jacobs, S. Kaul, W. K. Laskey, D. J. Pennell, J. A. Rumberger, T. Ryan, and M. S. Verani, "Standardized Myocardial Segmentation and Nomenclature for Tomographic Imaging of the Heart", Circulation, vol. 105, no. 4, pp. 539-542, Jan. 2002.

[60] A.K. Klein, F. Lee, A.A. Amini, "Quantitative coronary angiography with deformable spline models", Medical Imaging, IEEE Transactions, vol.16, no.5, pp.468-482, Oct. 1997.

[61] C. de Boor, A Practical Guide to Splines, Springer-Verlag, 1978.

[62] Bioscan Inc., NanoSPECT/CT Preclinical Imager;Technical Brochure and Specification Guide, Washington, DC.

[63] Siemens Medical Solutions USA, Inc., Inveon Systems Specifications, Hoffman Estates, IL, 2006.

[64] F. van der Have, B. Vastenhouw, R. M. Ramakers, W. Branderhorst, J. O. Krah, C. Ji, S. G. Staelens, and F. J. Beekman, "U-SPECT-II: An Ultra-High-Resolution Device for Molecular Small-Animal Imaging," Journal of Nuclear Medicine, vol. 50, no. 4, pp. 599-605, Apr. 2009.

[65] Y. Yang, J. Bec, J. Zhou, M. Zhang, M. Judenhofer, X. Bai, K. Shah, R. Farrell, J. Qi, and S. Cherry, "A prototype small-animal PET scanner dedicated to mouse brain imaging," Journal of Nuclear Medicine, vol. 53, no. 1, pp. 488, May 2012. 